TRANSACTIONS OF THE

AMERICAN MATHEMATICAL SOCIETY

Volume 351, Number 8, Pages 3143-3196

S 0002-9947(99)02441-1

Article electronically published on March 29, 1999

\title{
TOEPLITZ OPERATORS WITH PC SYMBOLS ON GENERAL CARLESON JORDAN CURVES WITH ARBITRARY MUCKENHOUPT WEIGHTS
}

\author{
ALBRECHT BÖTTCHER AND YURI I. KARLOVICH
}

\begin{abstract}
We describe the spectra and essential spectra of Toeplitz operators with piecewise continuous symbols on the Hardy space $H^{p}(\Gamma, \omega)$ in case $1<$ $p<\infty, \Gamma$ is a Carleson Jordan curve and $\omega$ is a Muckenhoupt weight in $A_{p}(\Gamma)$. Classical results tell us that the essential spectrum of the operator is obtained from the essential range of the symbol by filling in line segments or circular arcs between the endpoints of the jumps if both the curve $\Gamma$ and the weight are sufficiently nice. Only recently it was discovered by Spitkovsky that these line segments or circular arcs metamorphose into horns if the curve $\Gamma$ is nice and $\omega$ is an arbitrary Muckenhoupt weight, while the authors observed that certain special so-called logarithmic leaves emerge in the case of arbitrary Carleson curves with nice weights. In this paper we show that for general Carleson curves and general Muckenhoupt weights the sets in question are logarithmic leaves with a halo, and we present final results concerning the shape of the halo.
\end{abstract}

\section{INTRODUCTION}

Let $\Gamma$ be a Jordan curve in the complex plane, i.e. let $\Gamma$ be homeomorphic to a circle. Suppose $\Gamma$ is rectifiable and equip $\Gamma$ with Lebesgue length measure $|d \tau|$. A measurable function $\omega: \Gamma \rightarrow[0, \infty]$ is referred to as a weight if $\omega^{-1}(\{0, \infty\})$ has measure zero. We denote by $L^{p}(\Gamma)(1 \leq p \leq \infty)$ the usual Lebesgue spaces of $\Gamma$, and given a weight $\omega$ on $\Gamma$, we define $L^{p}(\Gamma, \omega)(1<p<\infty)$ as the Lebesgue space with the norm

$$
\|f\|_{p, \omega}:=\left(\int_{\Gamma}|f(\tau)|^{p} \omega^{p}(\tau)|d \tau|\right)^{1 / p} .
$$

As $\Gamma$ is a Jordan curve, it divides the plane into a bounded connected component $D_{+}$and an unbounded connected component $D_{-}$. We provide $\Gamma$ with counterclockwise orientation, that is, we demand that $D_{+}$stays on the left of $\Gamma$ when the curve is traced out in the positive direction.

Received by the editors September 28, 1995 and, in revised form, December 15, 1996.

1991 Mathematics Subject Classification. Primary 47B35; Secondary 30E20, 42A50, 45E05, $47 \mathrm{D} 30$.

Key words and phrases. Carleson condition, Ahlfors-David curve, Muckenhoupt condition, submultiplicative function, Toeplitz operator, singular integral operator, Fredholm operator.

The first author was supported by the Alfried Krupp Förderpreis für junge Hochschullehrer. Both authors were supported by NATO Collaborative Research Grant 950332.

(C)1999 American Mathematical Society 
The Cauchy singular integral. For a function $f \in L^{1}(\Gamma)$, the Cauchy singular integral is defined by

$$
(S f)(t):=\lim _{\varepsilon \rightarrow 0} \frac{1}{\pi i} \int_{\Gamma \backslash \Gamma(t, \varepsilon)} \frac{f(\tau)}{\tau-t} d \tau \quad(t \in \Gamma)
$$

where $\Gamma(t, \varepsilon):=\{\tau \in \Gamma:|\tau-t|<\varepsilon\}$ is the portion of $\Gamma$ contained in the open disk of radius $\varepsilon$ centered at $t$. The operator $S$ is said to be bounded on $L^{p}(\Gamma, \omega)$ if $L^{p}(\Gamma, \omega) \cap L^{1}(\Gamma)$ is dense in $L^{p}(\Gamma, \omega)$ and there exists a constant $M>0$ such that

$$
\|S f\|_{p, \omega} \leq M\|f\|_{p, \omega} \text { for all } L^{p}(\Gamma, \omega) \cap L^{1}(\Gamma) .
$$

The problem of characterizing the $\Gamma, p, \omega$ for which $S$ is bounded on $L^{p}(\Gamma, \omega)$ has been studied by many mathematicians for a long time. Here is the final result.

Theorem 1.1. Let $1<p<\infty$, let $\Gamma$ be a rectifiable Jordan curve, and let $\omega$ be a weight on $\Gamma$. The operator $S$ is bounded on $L^{p}(\Gamma, \omega)$ if and only if

$$
\omega \in L^{p}(\Gamma), \omega^{-1} \in L^{q}(\Gamma) \quad(1 / p+1 / q=1)
$$

and

$$
\sup _{t \in \Gamma} \sup _{\varepsilon>0}\left(\frac{1}{\varepsilon} \int_{\Gamma(t, \varepsilon)} \omega^{p}(\tau)|d \tau|\right)^{1 / p}\left(\frac{1}{\varepsilon} \int_{\Gamma(t, \varepsilon)} \omega^{-q}(\tau)|d \tau|\right)^{1 / q}<\infty .
$$

We remark that (1.1) implies that $L^{\infty}(\Gamma) \subset L^{p}(\Gamma, \omega) \subset L^{1}(\Gamma)$. The set of all weights $\omega$ on $\Gamma$ satisfying (1.1) and (1.2) is usually denoted by $A_{p}(\Gamma)$ and referred to as the set of Muckenhoupt weights. An easy application of Hölder's inequality shows that if (1.1) and (1.2) hold, then

$$
C_{\Gamma}:=\sup _{t \in \Gamma} \sup _{\varepsilon>0} \frac{|\Gamma(t, \varepsilon)|}{\varepsilon}<\infty
$$

where, of course, $|\Gamma(t, \varepsilon)|$ is the measure of the portion $\Gamma(t, \varepsilon)$. Condition (1.3) is a condition for solely the curve $\Gamma$, and curves $\Gamma$ satisfying (1.3) are commonly called Carleson curves. Although (1.3) is a consequence of (1.1) and (1.2), Theorem 1.1 is for psychological reasons often phrased in the following form:

$S$ is bounded on $L^{p}(\Gamma, \omega)(1<p<\infty)$ if and only if $\Gamma$ is a Carleson curve and $\omega$ is a Muckenhoupt weight.

In case $\Gamma$ is a smooth curve, Theorem 1.1 is due to Hunt, Muckenhoupt, and Wheeden [24]. Paatashvili and Khuskivadze [26] showed that $\Gamma$ is necessarily Carleson if $S$ is bounded on $L^{2}(\Gamma)$ and David [10], [11] proved that $S$ is bounded on $L^{2}(\Gamma)$ whenever $\Gamma$ is a Carleson curve. It was then observed by several authors (see e.g. [15] or [20], Vol. I, p. 50) that if $\Gamma$ is a Carleson curve, then $S$ is bounded on $L^{p}(\Gamma, \omega)$ if and only if $\omega \in A_{p}(\Gamma)$. In the form presented here, Theorem 1.1 was perhaps first stated in [13], [14], [15] (also see [22]); a proof of the fact that $S$ is bounded on $L^{2}(\Gamma)$ if it is bounded on $L^{p}(\Gamma, \omega)$ is contained in [4].

From now on we always suppose that $1<p<\infty$, that $\Gamma$ is a Carleson Jordan curve, and that $\omega \in A_{p}(\Gamma)$. Then $S$ is bounded on $L^{p}(\Gamma, \omega)$ and one can show that $S^{2}=I$ (see [20] and [4]). Hence, $P:=(I+S) / 2$ and $Q:=(I-S) / 2$ are bounded and complementary projections on $L^{p}(\Gamma, \omega)$. We define

$$
H^{p}(\Gamma, \omega):=P L^{p}(\Gamma, \omega), \dot{H}_{-}^{p}(\Gamma, \omega):=Q L^{p}(\Gamma, \omega), H_{-}^{p}(\Gamma, \omega):=\dot{H}_{-}^{p}(\Gamma, \omega)+\mathbf{C},
$$


where $\mathbf{C}$ stands for the constant functions on $\Gamma$. Since $\operatorname{Im} P=\operatorname{Ker} Q$ and $\operatorname{Im} Q=$ Ker $P$, the spaces $H^{p}(\Gamma, \omega), \dot{H}_{-}^{p}(\Gamma, \omega), H_{-}^{p}(\Gamma, \omega)$ are closed subspaces of $L^{p}(\Gamma, \omega)$. The space $H^{p}(\Gamma, \omega)$ is called the $p$ th Hardy space of $\Gamma$ and $\omega$.

Toeplitz operators. The Toeplitz operator $T(a)$ induced by a function $a \in L^{\infty}(\Gamma)$ is the bounded operator

$$
T(a): H^{p}(\Gamma, \omega) \rightarrow H^{p}(\Gamma, \omega), f \mapsto P(a f) .
$$

The function $a$ is in this context referred to as the symbol of the operator $T(a)$. Our main concern is the description of the spectrum of $T(a)$, i.e. of the set

$$
\operatorname{sp} T(a):=\left\{\lambda \in \mathbf{C}: T(a)-\lambda I \text { is not invertible on } H^{p}(\Gamma, \omega)\right\} .
$$

Toeplitz operators enjoy a particularly nice property: they are invertible if and only if they are Fredholm of index zero ("Coburn's lemma"); see e.g. [8], [30], [20], [4]. Recall that a bounded linear operator $A$ on a Banach space $X$ is said to be Fredholm if its image (= range) $\operatorname{Im} A$ is closed and its cokernel Coker $A:=X / \operatorname{Im} A$ and its kernel Ker $A:=\{f \in X: A f=0\}$ have finite dimensions. In that case the index of $A$ is defined as the integer Ind $A:=\operatorname{dim} \operatorname{Ker} A-\operatorname{dim}$ Coker $A$. Let $\mathcal{B}(X)$ be the Banach algebra of all bounded linear operators on $X$ and let $\mathcal{K}(X)$ stand for the ideal of compact operators on $X$. It is well known that $A \in \mathcal{B}(X)$ is Fredholm if and only if the coset $A+\mathcal{K}(X)$ is invertible in the quotient algebra $\mathcal{B}(X) / \mathcal{K}(X)$.

Since $T(a)-\lambda I=T(a-\lambda)$, we may deduce from Coburn's lemma that $\operatorname{sp} T(a)$ is the union of the essential spectrum of $T(a)$,

$$
\operatorname{sp}_{\mathrm{ess}} T(a):=\left\{\lambda \in \mathbf{C}: T(a)-\lambda I \text { is not Fredholm on } H^{p}(\Gamma, \omega)\right\},
$$

and of the set of all $\lambda \in \mathbf{C}$ for which $T(a)-\lambda I$ is Fredholm of nonzero index. As a rule, the latter set may be found without serious difficulty once only $\operatorname{sp}_{\text {ess }} T(a)$ is available.

Continuous symbols. Even in the case where $\Gamma$ is the complex unit circle $\mathbf{T}$, the weight $\omega$ is identically 1 , and $p=2$, there are no satisfactory descriptions of $\operatorname{sp} T(a)$ or $\operatorname{sp}_{\text {ess }} T(a)$ for general $a \in L^{\infty}(\Gamma)$; see [12], [20] or [6] for more about this topic. This motivates the consideration of symbols $a$ in certain subclasses of $L^{\infty}(\Gamma)$. For example, if $a$ is a continuous function on $\Gamma, a \in C(\Gamma)$, we have the following result.

Theorem 1.2. Suppose $1<p<\infty, \Gamma$ is a Carleson Jordan curve, $\omega \in A_{p}(\Gamma)$, and $a \in C(\Gamma)$. Then $\operatorname{sp}_{\text {ess }} T(a)=a(\Gamma)$. If $\lambda \notin a(\Gamma)$, then $\operatorname{Ind} T(a-\lambda)=-\operatorname{wind}(a-\lambda)$ where wind $(a-\lambda)$ denotes the winding number of the (naturally oriented) curve $a(\Gamma)$ about the point $\lambda$. Thus,

$$
\operatorname{sp} T(a)=a(\Gamma) \cup\{\lambda \in \mathbf{C} \backslash a(\Gamma): \operatorname{wind}(a-\lambda) \neq 0\} .
$$

This theorem goes back to Gohberg's 1952 paper [18] and was for general Carleson curves and general Muckenhoupt weights only recently proved in [4].

In the present paper, we determine $\operatorname{sp}_{\text {ess }} T(a)$ and $\operatorname{sp} T(a)$ for piecewise continuous symbols $a$ in case $\Gamma$ is an arbitrary Carleson curve and $\omega$ is an arbitrary Muckenhoupt weight. Section 2 contains a short history of the problem. The main results are stated and discussed in Section 3. The remaining Sections 4-10 are devoted to the proofs. 


\section{BRIEF HISTORY OF THE PROBLEM}

Piecewise continuous symbols. We denote by $P C(\Gamma)$ the closed algebra of all piecewise continuous functions on $\Gamma$ : a function $a \in L^{\infty}(\Gamma)$ belongs to $P C(\Gamma)$ if and only if the one-sided limits $a(t \pm 0)=\lim _{\tau \rightarrow t \pm 0} a(\tau)$ exist for every $t \in \Gamma$. Here $\tau \rightarrow t-0$ means that $\tau$ approaches $t$ following the orientation of $\Gamma$, while $\tau \rightarrow t+0$ says that $\tau$ goes to $t$ in the opposite direction. For $a \in P C(\Gamma)$, let $\Lambda_{a}$ denote the set of all points $t \in \Gamma$ at which $a$ has a jump,

$$
\Lambda_{a}:=\{t \in \Gamma: a(t-0) \neq a(t+0)\} .
$$

The set $\Lambda_{a}$ is at most countable and for each $\varepsilon>0$ the set of all $t$ in $\Gamma$ for which $|a(t+0)-a(t-0)|>\varepsilon$ is finite. Finally, let $\mathcal{R}(a)$ stand for the essential range of $a$,

$$
\mathcal{R}(a):=\bigcup_{t \in \Gamma}\{a(t-0), a(t+0)\}=a\left(\Gamma \backslash \Lambda_{a}\right) \cup \bigcup_{t \in \Lambda_{a}}\{a(t-0), a(t+0)\} ;
$$

here, for $t \in \Gamma \backslash \Lambda_{a}$, we understand by $a(t)$ the common value of $a(t-0)$ and $a(t+0)$.

The primary matter: line segments. The story of describing $\operatorname{sp}_{\text {ess }} T(a)$ for $a$ in $P C(\Gamma)$ has its beginning in the sixties, when several mathematicians, including I.B. Simonenko, A. Calderón, F. Spitzer, H. Widom, A. Devinatz, I. Gohberg, and N. Krupnik, realized that if $\Gamma$ is a piecewise smooth curve, then the essential spectrum of $T(a)$ on $H^{2}(\Gamma)$ is the closed continuous curve resulting from the essential range of $a$ by filling in a line segment between the endpoints of each jump:

$$
\operatorname{sp}_{\text {ess }} T(a)=\mathcal{R}(a) \cup \bigcup_{t \in \Lambda_{a}}[a(t-0), a(t+0)] .
$$

Metamorphosis 1: circular arcs. The first surprise came with the consideration of the case $p \neq 2$. Widom [34] as well as Gohberg and Krupnik [19] observed that then the line segments mentioned above go over into circular arcs. Given two points $z, w \in \mathbf{C}$ and a number $\nu \in(0,1)$, define

$$
\mathcal{A}(z, w ; \nu):=\left\{\lambda \in \mathbf{C} \backslash\{z, w\}: \arg \frac{\lambda-z}{\lambda-w} \in 2 \pi \nu+2 \pi \mathbf{Z}\right\} \cup\{z, w\} .
$$

A moment's thought reveals that $\mathcal{A}(z, w ; \nu)$ is a circular arc between $z$ and $w$ whose shape is determined by $\nu$. For our further purposes, it is convenient to think of $\mathcal{A}(z, w ; \nu)$ as given in the following way. Denote by $Y_{\nu}$ the horizontal line $Y_{\nu}=\{\gamma \in \mathbf{C}: \operatorname{Im} \gamma=\nu\}$. Then $\left\{e^{2 \pi \gamma}: \gamma \in Y_{\nu}\right\}$ is a ray starting at the origin and making the angle $2 \pi \nu$ with the real axis. Let $M_{z, w}(\zeta):=(w \zeta-z) /(\zeta-1)$. Clearly, $M_{z, w}$ is a Möbius transformation mapping 0 and $\infty$ to $z$ and $w$, respectively. So we may write

$$
\mathcal{A}(z, w ; \nu)=\left\{M_{z, w}\left(e^{2 \pi \gamma}\right): \gamma \in Y_{\nu}\right\} \cup\{z, w\} .
$$

Notice that $\mathcal{A}(z, w ; 1 / 2)$ is nothing but the line segment $[z, w]$. The Widom/Gohberg/Krupnik result says that if $\Gamma$ is piecewise smooth, $\omega$ is identically $1,1<p<\infty$, and $a \in P C(\Gamma)$, then

$$
\operatorname{sp}_{\mathrm{ess}} T(a)=\mathcal{R}(a) \cup \bigcup_{t \in \Lambda_{a}} \mathcal{A}\left(a(t-0), a(t+0) ; \frac{1}{p}\right) .
$$


Gohberg and Krupnik (see [19] and [20]) also studied spaces with so-called power weights, that is, with weights of the form

$$
\omega(\tau)=\prod_{j=1}^{n}\left|\tau-t_{j}\right|^{\lambda_{j}} \quad(\tau \in \Gamma)
$$

where $t_{1}, \ldots, t_{n}$ are distinct points on $\Gamma$ and $\lambda_{1}, \ldots, \lambda_{n}$ are nonzero real numbers. The weight (2.1) belongs to $A_{p}(\Gamma)$ if and only if $-1 / p<\lambda_{j}<1 / q$ for all $j$. This result has been well known for a long time under several additional hypotheses and was obtained in the work of G.H. Hardy, J.E. Littlewood, M. Riesz, S.G. Mikhlin, K.I. Babenko, B.V. Khvedelidze, H. Helson, G. Szegö, H. Widom, F. Forelli, I.I. Danilyuk, V.Yu. Shelepov, A.P. Calderón, and others (see, e.g., [15]). For general Carleson curves a proof was first given by E.A. Danilov in [9]; his proof is reproduced in [4]. Gohberg and Krupnik showed that for piecewise smooth curves with the weight (2.1) one has

$$
\mathrm{sp}_{\mathrm{ess}} T(a)=\mathcal{R}(a) \cup \bigcup_{t \in \Lambda_{a}} \mathcal{A}\left(a(t-0), a(t+0) ; \frac{1}{p}+\lambda_{t}\right)
$$

where $\lambda_{t}=0$ for $t \notin\left\{t_{1}, \ldots, t_{n}\right\}$ and $\lambda_{t_{j}}=\lambda_{j}$. Thus, although now the circular arcs participating in the spectrum may have different shape, they nevertheless remain circular arcs.

Metamorphosis 2: horns. The development had paused many years until 1990, when Spitkovsky [33] made a spectacular discovery. He considered again the case of a piecewise smooth curve $\Gamma$, but he admitted arbitrary Muckenhoupt weights $\omega \in A_{p}(\Gamma)(1<p<\infty)$. His result says that the presence of Muckenhoupt weights may metamorphose the circular arcs into so-called horns. A horn is a closed subset of the plane which is bounded by two circular arcs. Given two numbers $\mu, \nu \in(0,1)$ satisfying $\mu \leq \nu$, denote by $Y_{\mu, \nu}$ the closed stripe between the horizontal lines through $i \mu$ and $i \nu$, i.e. $Y_{\mu, \nu}=\{\gamma \in \mathbf{C}: \mu \leq \operatorname{Im} \gamma \leq \nu\}$. Then $\left\{e^{2 \pi \gamma}: \gamma \in Y_{\mu, \nu}\right\}$ is an angular sector with the vertex at the origin. With $M_{z, w}(\zeta)=(w \zeta-z) /(\zeta-1)$ as above, put

$$
\mathcal{H}(z, w ; \mu, \nu):=\left\{M_{z, w}\left(e^{2 \pi \gamma}\right): \gamma \in Y_{\mu, \nu}\right\} \cup\{z, w\} .
$$

Thus, $\mathcal{H}(z, w ; \mu, \nu)$ is the horn between $z$ and $w$ whose boundary arcs are $\mathcal{A}(z, w ; \mu)$ and $\mathcal{A}(z, w ; \nu)$; see Figure 1. Spitkovsky associated two numbers $\mu_{t}$ and $\nu_{t}$ with each point $t \in \Gamma$ which, in a sense, measure the "powerlikeness" of the weight $\omega$ at $t$ and proved that

$$
\operatorname{sp}_{\text {ess }} T(a)=\mathcal{R}(a) \cup \bigcup_{t \in \Lambda_{a}} \mathcal{H}\left(a(t-0), a(t+0) ; \frac{1}{p}+\mu_{t}, \frac{1}{p}+\nu_{t}\right) .
$$

Metamorphosis 3: spiralic horns. In 1994, partially stimulated by the results of [1] and [2], we turned attention to the case where $\Gamma$ is no longer supposed to be piecewise smooth but is allowed to be a more complicated Carleson Jordan curve [4]. The class of Carleson curves considered in [4] is as follows. Fix a point $t \in \Gamma$. We then have $\tau-t=|\tau-t| e^{i \arg (\tau-t)}$ for $\tau \in \Gamma \backslash\{t\}$, and the $\operatorname{argument} \arg (\tau-t)$ may be chosen so that it is a continuous function on $\Gamma \backslash\{t\}$. Seifullayev [28] showed that for an arbitrary Carleson Jordan curve the estimate

$$
\arg (\tau-t)=O(-\log |\tau-t|) \text { as } \tau \rightarrow t
$$



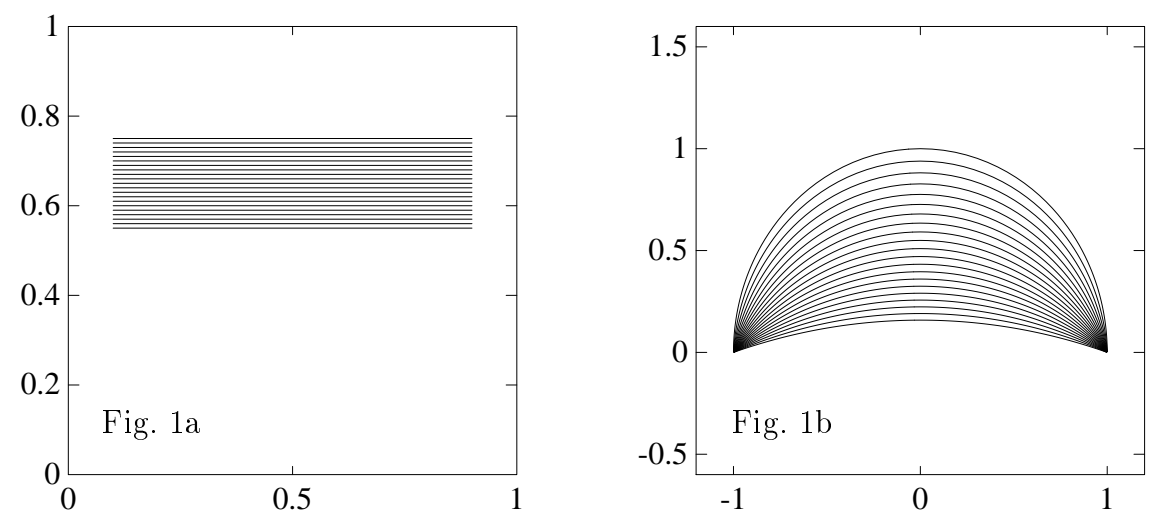

Figure 1. Figure 1a shows part of the horizontal stripe $Y_{0.55,0.75}$; the horn $\mathcal{H}(-1,1 ; 0.55,0.75)$ is plotted in Figure $1 \mathrm{~b}$.

holds. In [4], we studied curves with the property that for each $t \in \Gamma$ there is a $\delta_{t} \in \mathbf{R}$ such that

$$
\arg (\tau-t)=-\delta_{t} \log |\tau-t|+O(1) \text { as } \tau \rightarrow t .
$$

Points $t$ at which $(2.3)$ is valid with $\delta_{t}=0$ are "nonhelical" points. In particular, points at which the tangent exists, corner points, cusp points, or points at which the argument oscillates but remains bounded are "nonhelical" points. If

$$
\arg (\tau-t)=-\delta_{t} \log |\tau-t|+C_{t}^{ \pm} \text {as } \tau \rightarrow t \pm 0,
$$

where $C_{t}^{ \pm}$are real numbers such that $C_{t}^{+}-C_{t}^{-} \notin 2 \pi \mathbf{Z}$, then in a neighborhood of $t$ the curve $\Gamma$ consists of two logarithmic spirals scrolling up at $t$. A Carleson Jordan curve satisfying (2.3) at each point $t$ will be called a spiralic curve.

The main result of [4] implies that for spiralic curves the circular arcs and horns may change into logarithmic double-spirals and spiralic horns. Let $\mu, \nu, \delta$ be real numbers such that $0<\mu \leq \nu<1$. Define the straight line $Y_{\nu}^{\delta}$ and the stripe $Y_{\mu, \nu}^{\delta}$ by

$$
\begin{aligned}
& Y_{\nu}^{\delta}:=\{\gamma \in \mathbf{C}: \operatorname{Im} \gamma=\delta \operatorname{Re} \gamma+\nu\}, \\
& Y_{\mu, \nu}^{\delta}:=\{\gamma \in \mathbf{C}: \delta \operatorname{Re} \gamma+\mu \leq \operatorname{Im} \gamma \leq \delta \operatorname{Re} \gamma+\nu\} .
\end{aligned}
$$

We already know that $\left\{e^{2 \pi \gamma}: \gamma \in Y_{\nu}^{\delta}\right\}$ is a ray starting at the origin in case $\delta=0$. If $\delta \neq 0$, then $\left\{e^{2 \pi \gamma}: \gamma \in Y_{\nu}^{\delta}\right\}$ is a logarithmic spiral wriggling out of the origin. Thus, $\left\{e^{2 \pi \gamma}: \gamma \in Y_{\mu, \nu}^{\delta}\right\}$ is the closed set between two such logarithmic spirals. Again let $M_{z, w}(\zeta)=(w \zeta-z) /(\zeta-1)$. Put

$$
\begin{aligned}
& \mathcal{S}(z, w ; \delta ; \nu):=\left\{M_{z, w}\left(e^{2 \pi \gamma}\right): \gamma \in Y_{\nu}^{\delta}\right\} \cup\{z, w\}, \\
& \mathcal{S}(z, w ; \delta ; \mu, \nu):=\left\{M_{z, w}\left(e^{2 \pi \gamma}\right): \gamma \in Y_{\mu, \nu}^{\delta}\right\} \cup\{z, w\} .
\end{aligned}
$$

Thus, if $\delta \neq 0$, then $\mathcal{S}(z, w ; \delta ; \nu)$ is a double-spiral coming out of $z$ and scrolling up at $w$, while $\mathcal{S}(z, w ; \delta ; \mu, \nu)$ is the closed set between two such double-spirals. We call $\mathcal{S}(z, w ; \delta ; \nu)$ a logarithmic double-spiral and $\mathcal{S}(z, w ; \delta ; \mu, \nu)$ a spiralic horn; see Figure 2 .

In case $\delta=0$, logarithmic double-spirals degenerate to circular $\operatorname{arcs}(\nu \neq 1 / 2)$ or line segments $(\nu=1 / 2)$, and spiralic horns become usual horns. In [4] we showed 

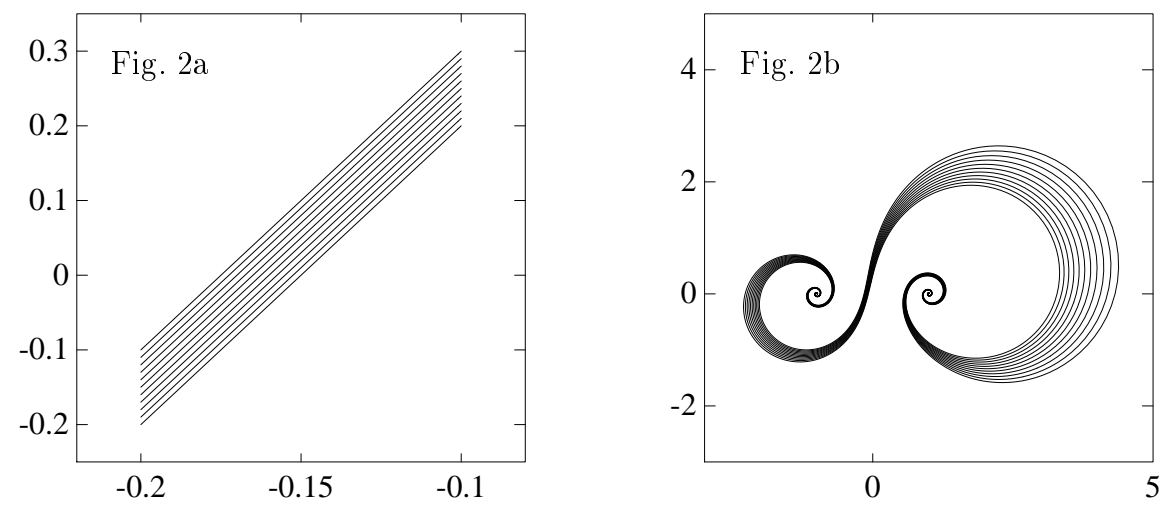

Figure 2. Figure 2a shows a piece of the stripe between the lines $y=0.6+4 x$ and $y=0.7+4 x$; the spiralic horn $\mathcal{S}(-1,1 ; 4 ; 0.6,0.7)$ is shown in Figure $2 \mathrm{~b}$.

that if $\Gamma$ is a spiralic curve, $\omega \in A_{p}(\Gamma)(1<p<\infty)$, and $a \in P C(\Gamma)$, then

$$
\operatorname{sp}_{\mathrm{ess}} T(a)=\mathcal{R}(a) \cup \bigcup_{t \in \Lambda_{a}} \mathcal{S}\left(a(t-0), a(t+0) ; \delta_{t} ; \frac{1}{p}+\mu_{t}, \frac{1}{p}+\nu_{t}\right)
$$

where $\delta_{t}$ is given by $(2.3)$ and $\mu_{t}, \nu_{t}$ are real numbers satisfying $-1 / p<\mu_{t} \leq \nu_{t}<$ $1 / q$ and measuring the "powerlikeness" of $\omega$ at $t$. We remark that for weights of the form (2.1) one has $\mu_{t}=\nu_{t}=\lambda_{t}$ with $\lambda_{t}=0$ for $t \notin\left\{t_{1}, \ldots, t_{n}\right\}$ and $\lambda_{t_{j}}=\lambda_{j}$.

Metamorphosis 4: appearance of logarithmic leaves. In [5] we disposed of the case of a general Carleson Jordan curve $\Gamma$ without weight, i.e. we assumed that $\omega(\tau)=1$ for all $\tau \in \Gamma$. We know from the preceding paragraph that in the absence of a weight we have to fill in logarithmic double-spirals between the endpoints of the jumps if $\Gamma$ is a spiralic curve. In [5] we proved that for general curves these logarithmic double-spirals may blow up to certain sets which in [5] were somewhat insuggestively called skew spiralic horns. In the terminology that will be introduced in the next section, these sets may be characterized as logarithmic leaves with a separating point at an equal distance to $a(t-0)$ and $a(t+0)$. For each point $t \in \Gamma$, we defined two numbers $\delta_{t}^{-} \leq \delta_{t}^{+}$which measure, in a sense, the "spirality" of the curve $\Gamma$ at $t$. If (2.3) holds, it turns out that $\delta_{t}^{-}=\delta_{t}^{+}=\delta_{t}$. We proved that if $\Gamma$ is an arbitrary Carleson Jordan curve, $1<p<\infty$, and $a \in P C(\Gamma)$, then the essential spectrum of $T(a)$ on $H^{p}(\Gamma)$ is given by

$$
\operatorname{sp}_{\mathrm{ess}} T(a)=\mathcal{R}(a) \cup \bigcup_{t \in \Lambda_{a}}\left(\bigcup_{\delta \in\left[\delta_{t}^{-}, \delta_{t}^{+}\right]} \mathcal{S}\left(a(t-0), a(t+0) ; \delta ; \frac{1}{p}\right)\right)
$$

where $\mathcal{S}(z, w ; \delta ; \nu)$ is again defined by $(2.4)$. In the notation of the next section, we have

$$
\bigcup_{\delta \in\left[\delta_{t}^{-}, \delta_{t}^{+}\right]} \mathcal{S}\left(z, w ; \delta ; \frac{1}{p}\right)=\mathcal{L}^{0}\left(z, w ; \delta_{t}^{-}, \delta_{t}^{+} ; \frac{1}{p}, \frac{1}{p}, \frac{1}{p}, \frac{1}{p}\right) .
$$



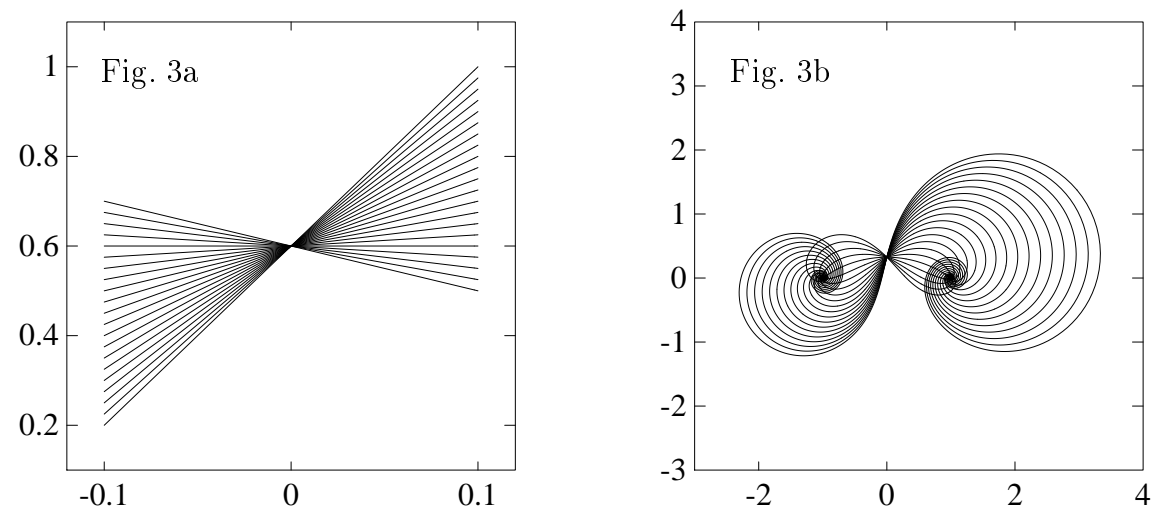

Figure 3. The set (2.8) may be represented as $\left\{M_{z, w}\left(e^{2 \pi \gamma}\right): \gamma \in\right.$ $\Delta$ \} where $\Delta$ is the double-sector filled out by the straight lines $y=1 / p+\delta x$ with $\delta \in\left[\delta_{t}^{-}, \delta_{t}^{+}\right]$. In Figure 3 a we plotted this double-sector for $1 / p=0.6, \delta_{t}^{-}=-1, \delta_{t}^{+}=4$. The corresponding set (2.8) with $z=-1, w=1$ is shown in Figure 3b.

If $\delta_{t}^{-}=\delta_{t}^{+}$, then $(2.8)$ is simply a logarithmic double-spiral. So let $\delta_{t}^{-}<\delta_{t}^{+}$. Then the set (2.8) is easily seen to be a heavy set, i.e. a set with a nonempty interior. In particular, the points $z$ and $w$ are inner points; see Figure 3.

All logarithmic double-spirals $\mathcal{S}(z, w ; \delta ; 1 / p)$ contain the point

$$
s:=\left(w e^{2 \pi i / p}-z\right) /\left(e^{2 \pi i / p}-1\right) .
$$

The set (2.8) is connected, while (2.8) minus the point $s$ is disconnected. This is why we call the point $s$ a separating point. Since

$$
|s-z|=|s-w|=|w-z| /\left(2 \sin \frac{\pi}{p}\right),
$$

the point $s$ is at an equal distance to $z$ and $w$.

Metamorphosis 5: the subject of this paper. In all the cases we have considered so far, spiralic curves with general weights and general curves with no weight, the sets we had to fill in between the points $a(t-0)$ and $a(t+0)$ were bounded by pieces of at most two logarithmic double-spirals (let us think of circular arcs and line segments as degenerate logarithmic double-spirals). In the case of a spiralic curve with a general weight these two logarithmic double-spirals are determined by two numbers $\mu_{t}, \nu_{t}$ measuring the "powerlikeness" of the weight, whereas in the case of a general curve with no weight these two logarithmic double-spirals are given by two numbers $\delta_{t}^{-}, \delta_{t}^{+}$measuring the "spirality" of the curve. One might conjecture that in the case of a general curve and a general weight the set in question is bounded by pieces of at most four logarithmic double-spirals depending on $\mu_{t}, \nu_{t}, \delta_{t}^{-}, \delta_{t}^{+}$. We will show that this is in general not true. There may appear some kind of interference between the oscillation of the curve and the oscillation of the weight which metamorphoses logarithmic double-spirals into qualitatively new curves. 


\section{MAin RESUlts}

Throughout what follows, $\Gamma$ is a Carleson Jordan curve, $p$ is a number in $(1, \infty)$, and $\omega \in A_{p}(\Gamma)$ is a Muckenhoupt weight.

Leaves. We will construct two families $\left\{\alpha_{t}\right\}_{t \in \Gamma}$ and $\left\{\beta_{t}\right\}_{t \in \Gamma}$ of continuous functions $\alpha_{t}: \mathbf{R} \rightarrow \mathbf{R}$ and $\beta_{t}: \mathbf{R} \rightarrow \mathbf{R}$ such that

$$
\alpha_{t}(x) \leq \beta_{t}(x) \text { for all } x \in \mathbf{R}
$$

which will be called the indicator functions of $\Gamma, p, \omega$ and in terms of which the essential spectrum of Toeplitz operators on $H^{p}(\Gamma, \omega)$ will be described. Given $\alpha_{t}$ and $\beta_{t}$, we define

$$
Y\left(p, \alpha_{t}, \beta_{t}\right):=\left\{\gamma=x+i y \in \mathbf{C}: \frac{1}{p}+\alpha_{t}(x) \leq y \leq \frac{1}{p}+\beta_{t}(x)\right\},
$$

and for $z, w \in \mathbf{C}$ we then put

$$
\mathcal{L}\left(z, w ; p, \alpha_{t}, \beta_{t}\right):=\left\{M_{z, w}\left(e^{2 \pi \gamma}\right): \gamma \in Y\left(p, \alpha_{t}, \beta_{t}\right)\right\} \cup\{z, w\}
$$

where $M_{z, w}(\zeta):=(w \zeta-z) /(\zeta-1)$. We refer to $\mathcal{L}\left(z, w ; p, \alpha_{t}, \beta_{t}\right)$ as the leaf about $z$ and $w$ determined by $p, \alpha_{t}, \beta_{t}$. Our main result is as follows.

Theorem 3.1. If $a \in P C(\Gamma)$, then the essential spectrum of the Toeplitz operator $T(a)$ on the Hardy space $H^{p}(\Gamma, \omega)$ is given by

$$
\operatorname{sp}_{\mathrm{ess}} T(a)=\mathcal{R}(a) \cup \bigcup_{t \in \Lambda_{a}} \mathcal{L}\left(a(t-0), a(t+0) ; p, \alpha_{t}, \beta_{t}\right) .
$$

If $\lambda \notin \mathrm{sp}_{\text {ess }} T(a)$, then the index of $T(a)-\lambda I$ equals minus the winding number of the closed, continuous, and naturally oriented curve

$$
\mathcal{R}(a) \cup \bigcup_{t \in \Lambda_{a}} \mathcal{L}\left(a(t-0), a(t+0) ; p, \frac{\alpha_{t}+\beta_{t}}{2}, \frac{\alpha_{t}+\beta_{t}}{2}\right)
$$

about the point $\lambda$.

By virtue of (3.1), this theorem implies in particular that $\mathrm{sp}_{\text {ess }} T(a)$ is connected for every $a \in P C(\Gamma)$. We also remark that once (3.2) is established, the assertion concerning the index can be derived from Theorem 1.1 by standard homotopy arguments.

Indicator functions. We now proceed to the definition of the indicator functions. Fix $t \in \Gamma$. Given a weight $\psi: \Gamma \rightarrow[0, \infty]$ for which $\log \psi \in L^{1}(\Gamma)$, we define a new function $V_{t}^{0} \psi:(0, \infty) \rightarrow[0, \infty]$ by

$$
\left(V_{t}^{0} \psi\right)(\xi):=\left\{\begin{array}{l}
\limsup _{R \rightarrow 0} \frac{\exp \left(\int_{\Gamma(t, \xi R)} \log \psi(\tau)|d \tau| /|\Gamma(t, \xi R)|\right)}{\exp \left(\int_{\Gamma(t, R)}^{\int} \log \psi(\tau)|d \tau| /|\Gamma(t, R)|\right)} \text { for } \xi \in(0,1], \\
\limsup _{R \rightarrow 0} \frac{\exp \left(\int_{\Gamma(t, R)}^{\int} \log \psi(\tau)|d \tau| /|\Gamma(t, R)|\right)}{\exp \left(\int_{\Gamma\left(t, \xi^{-1} R\right)}^{\int} \log \psi(\tau)|d \tau| /\left|\Gamma\left(t, \xi^{-1} R\right)\right|\right)} \text { for } \xi \in[1, \infty),
\end{array}\right.
$$

and then we put

$$
\alpha\left(V_{t}^{0} \psi\right):=\limsup _{\xi \rightarrow 0} \frac{\log \left(V_{t}^{0} \psi\right)(\xi)}{\log \xi}, \beta\left(V_{t}^{0} \psi\right):=\limsup _{\xi \rightarrow \infty} \frac{\log \left(V_{t}^{0} \psi\right)(\xi)}{\log \xi} .
$$


For $\tau \in \Gamma$ we have $\tau-t=|\tau-t| e^{i \arg (\tau-t)}$, and $\arg (\tau-t)$ may be chosen as a continuous function of $\tau \in \Gamma \backslash\{t\}$. We write

$$
\eta_{t}(\tau):=e^{-\arg (\tau-t)} \text { for } \tau \in \Gamma \backslash\{t\}
$$

Since $-\log |\tau-t|<|\tau-t|^{-1 / 2}$ for all $\tau \in \Gamma \backslash\{t\}$, the function $-\log |\tau-t|$ belongs to $L^{1}(\Gamma)$. We so infer from (2.2) that $\log \eta_{t} \in L^{1}(\Gamma)$. It is easily seen (Lemma 5.3) that $\log \omega \in L^{1}(\Gamma)$ whenever (1.1) holds. Therefore $\log \left(\eta_{t}^{x} \omega\right)=x \log \eta_{t}+\log \omega \in L^{1}(\Gamma)$ for $x \in \mathbf{R}$, and we may carry out the above construction with $\psi=\eta_{t}^{x} \omega$ to obtain

$$
\alpha_{t}(x):=\alpha\left(V_{t}^{0} \eta_{t}^{x} \omega\right), \beta_{t}(x):=\beta\left(V_{t}^{0} \eta_{t}^{x} \omega\right) .
$$

Theorem 3.2. The functions given by (3.4) enjoy the following properties:

(a) $-\infty<\alpha_{t}(x) \leq \beta_{t}(x)<+\infty$ for all $x \in \mathbf{R}$;

(b) $-1 / p<\alpha_{t}(0) \leq \beta_{t}(0)<1 / q$;

(c) $\alpha_{t}$ is a concave function and $\beta_{t}$ is a convex function;

(d) $\alpha_{t}(x)$ and $\beta_{t}(x)$ have asymptotes as $x \rightarrow \pm \infty$, and the (convex) regions $\left\{x+i y \in \mathbf{C}: y<\alpha_{t}(x)\right\}$ and $\left\{x+i y \in \mathbf{C}: y>\beta_{t}(x)\right\}$ may be separated by parallels to these asymptotes; more precisely, there exist real numbers $\delta_{t}^{-}, \delta_{t}^{+}, \mu_{t}^{-}, \mu_{t}^{+}, \nu_{t}^{-}, \nu_{t}^{+}$ such that

$$
\delta_{t}^{-} \leq \delta_{t}^{+},-1 / p<\mu_{t}^{-} \leq \nu_{t}^{-}<1 / q,-1 / p<\mu_{t}^{+} \leq \nu_{t}^{+}<1 / q
$$

and

$$
\begin{aligned}
& \beta_{t}(x)=\nu_{t}^{+}+\delta_{t}^{+} x+o(1) \text { as } x \rightarrow+\infty, \\
& \beta_{t}(x)=\nu_{t}^{-}+\delta_{t}^{-} x+o(1) \text { as } x \rightarrow-\infty, \\
& \alpha_{t}(x)=\mu_{t}^{+}+\delta_{t}^{+} x+o(1) \text { as } x \rightarrow-\infty, \\
& \alpha_{t}(x)=\mu_{t}^{-}+\delta_{t}^{-} x+o(1) \text { as } x \rightarrow+\infty ;
\end{aligned}
$$

(e) Theorem 3.1 is true with $\alpha_{t}$ and $\beta_{t}$ given by (3.4).

Thus, by property (e), $\alpha_{t}$ and $\beta_{t}$ are the indicator functions. We remark that the continuity of $\alpha_{t}$ and $\beta_{t}$ is a consequence of (c) (see [27], Corollary 10.1.1).

Spiralic horns. First let $\delta_{t}^{-}=\delta_{t}^{+}=: \delta_{t}$. Then, by $(\mathrm{d}), \beta_{t}(x)-\alpha_{t}(x)$ has finite limits as $x \rightarrow \pm \infty$, and since, by (c), $\beta_{t}-\alpha_{t}$ is convex, it follows that $\beta_{t}-\alpha_{t}$ must be a constant:

$$
\beta_{t}(x)=\alpha_{t}(x)+c \text { for all } x \in \mathbf{R} .
$$

Combining (3.5) with (c) we see that $\alpha_{t}$ and $\beta_{t}$ are simultaneously convex and concave, which implies that they are affine linear. Now (d) shows that actually

$$
\alpha_{t}(x)=\mu_{t}+\delta_{t} x, \beta_{t}(x)=\nu_{t}+\delta_{t} x
$$

with

$$
\mu_{t}:=\mu_{t}^{-}=\mu_{t}^{+}=\alpha_{t}(0), \nu_{t}:=\nu_{t}^{-}=\nu_{t}^{+}=\beta_{t}(0) .
$$

From (b) we infer that $-1 / p<\mu_{t} \leq \nu_{t}<1 / q$. Thus, (3.6) yields that, in the case at hand,

$$
\mathcal{L}\left(z, w ; p, \alpha_{t}, \beta_{t}\right)=\mathcal{S}\left(z, w ; \delta_{t} ; \frac{1}{p}+\mu_{t}, \frac{1}{p}+\nu_{t}\right)
$$


where $\mathcal{S}\left(z, w ; \delta_{t} ; 1 / p+\mu_{t}, 1 / p+\nu_{t}\right)$ is the spiralic horn given by $(2.5)$; if even $\mu_{t}=\nu_{t}$, this spiralic horn degenerates to a logarithmic double-spiral.

We remark that (3.6) and (b) imply that $\beta_{t}(x)-\alpha_{t}(x)<1$ for all $x \in \mathbf{R}$ whenever $\delta_{t}^{-}=\delta_{t}^{+}$. Also notice that the stripes in Figures 1a and 2a are the stripes between the lines $y=1 / p+\mu_{t}+\delta_{t} x$ and $y=1 / p+\nu_{t}+\delta_{t} x$ (with $\delta_{t}=0$ in Figure 1a).

Shape of general leaves. Now suppose $\delta_{t}^{-}<\delta_{t}^{+}$. Then $\beta_{t}(x)-\alpha_{t}(x)$ is a convex function of $x$ which is less than 1 for $x=0$ (by (b)) and increases to infinity as $x \rightarrow \pm \infty$ (by (d)). Hence, the equation

$$
\beta_{t}(x)-\alpha_{t}(x)=1
$$

has exactly two solutions $x_{t}^{-}$and $x_{t}^{+}$. Obviously, $x_{t}^{-}<0$ and $x_{t}^{+}>0$. Denote by $\Pi_{t}:=\Pi_{t}(\Gamma, p, \omega)$ the closed parallelogram spanned by the four points

$$
\begin{aligned}
& x_{t}^{-}+i\left(\frac{1}{p}+\alpha_{t}\left(x_{t}^{-}\right)\right), x_{t}^{-}+i\left(\frac{1}{p}+\beta_{t}\left(x_{t}^{-}\right)\right), \\
& x_{t}^{+}+i\left(\frac{1}{p}+\alpha_{t}\left(x_{t}^{+}\right)\right), x_{t}^{+}+i\left(\frac{1}{p}+\beta_{t}\left(x_{t}^{+}\right)\right)
\end{aligned}
$$

and let $\Pi_{t}^{ \pm}:=\Pi_{t}^{ \pm}(\Gamma, p, \omega)$ stand for the horizontal half-stripes

$$
\begin{aligned}
& \Pi_{t}^{-}:=\left\{x+i y \in \mathbf{C}: x \leq x_{t}^{-}, \frac{1}{p}+\alpha_{t}\left(x_{t}^{-}\right) \leq y \leq \frac{1}{p}+\beta_{t}\left(x_{t}^{-}\right)\right\}, \\
& \Pi_{t}^{+}:=\left\{x+i y \in \mathbf{C}: x \geq x_{t}^{+}, \frac{1}{p}+\alpha_{t}\left(x_{t}^{+}\right) \leq y \leq \frac{1}{p}+\beta_{t}\left(x_{t}^{+}\right)\right\} .
\end{aligned}
$$

The map $\gamma \mapsto M_{z, w}\left(e^{2 \pi \gamma}\right)$ has the period $i$, and hence

$$
\begin{aligned}
& \mathcal{L}\left(z, w ; p, \alpha_{t}, \beta_{t}\right)=\left\{M_{z, w}\left(e^{2 \pi \gamma}\right): \gamma \in \Pi_{t}^{-} \cup \Pi_{t}^{+}\right\} \\
& \quad \cup\left\{M_{z, w}\left(e^{2 \pi \gamma}\right): \gamma=x+i y \in \Pi_{t}, \frac{1}{p}+\alpha_{t}(x) \leq y \leq \frac{1}{p}+\beta_{t}(x)\right\} \cup\{z, w\} .
\end{aligned}
$$

Clearly, $\left\{M_{z, w}\left(e^{2 \pi \gamma}\right): \gamma \in \Pi_{t}^{-} \cup \Pi_{t}^{+}\right\}$is a set consisting of two disks (punctured at $z$ and $w$ ). The second set on the right of (3.9) is something linking these two disks and therefore the portion of $Y\left(p, \alpha_{t}, \beta_{t}\right)$ contained in the parallelogram $\Pi_{t}$ is the actually interesting part of $Y\left(p, \alpha_{t}, \beta_{t}\right)$; see Figure 4 .

Theorem 3.3. If the equation (3.8) has exactly two solutions $x_{t}^{-}$and $x_{t}^{+}$, then the indicator functions $\alpha_{t}$ and $\beta_{t}$ possess the following properties:

$\left(P_{1}\right) \beta_{t}\left(x_{t}^{-}\right)-\alpha_{t}\left(x_{t}^{-}\right)=1, \beta_{t}\left(x_{t}^{+}\right)-\alpha_{t}\left(x_{t}^{+}\right)=1$;

$\left(P_{2}\right)-1 / p<\alpha_{t}(0) \leq \beta_{t}(0)<1 / q$;

$\left(P_{3}\right) \alpha_{t}$ is concave and $\beta_{t}$ is convex on $\left[x_{t}^{-}, x_{t}^{+}\right]$;

$\left(P_{4}\right)$ each diagonal of the parallelogram $\Pi_{t}$ spanned by the points (3.9) separates the (convex) regions

$$
\left\{x+i y \in \mathbf{C}: x_{t}^{-}<x<x_{t}^{+}, y<\frac{1}{p}+\alpha_{t}(x)\right\}
$$

and

$$
\left\{x+i y \in \mathbf{C}: x_{t}^{-}<x<x_{t}^{+}, y>\frac{1}{p}+\beta_{t}(x)\right\} \text {. }
$$



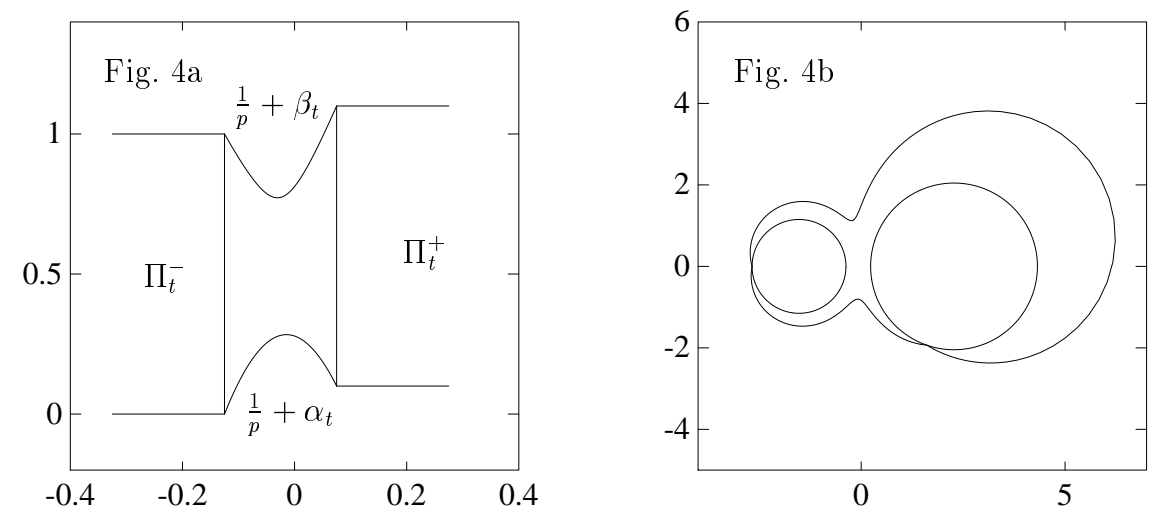

Figure 4. Figure 4a shows the two half-stripes $\Pi_{t}^{-}$and $\Pi_{t}^{+}$as well as the graphs of $y=1 / p+\alpha_{t}(x)$ and $y=1 / p+\beta_{t}(x)$ for $x \in\left[x_{t}^{-}, x_{t}^{+}\right]$. In Figure $4 \mathrm{~b}$ we see the two disks $\left\{M_{z, w}\left(e^{2 \pi \gamma}\right): \gamma \in\right.$ $\left.\Pi_{t}^{-} \cup \Pi_{t}^{+}\right\}$and the "linking set" given by (3.9).

This theorem can be without difficulty derived from Theorem 3.2 and the definition of the indicator functions. The following theorem is significantly less trivial.

Theorem 3.4. Given any number $p \in(1, \infty)$, any numbers $x_{t}^{-} \in(-\infty, 0), x_{t}^{+} \in$ $(0, \infty)$, and any functions $\alpha_{t}$ and $\beta_{t}$ on $\left[x_{t}^{-}, x_{t}^{+}\right]$with the properties $\left(P_{1}\right)-\left(P_{4}\right)$, there exist a Carleson Jordan curve $\Gamma$, a point $t \in \Gamma$, and a weight $\omega \in A_{p}(\Gamma)$ such that the equation $\beta\left(V_{t}^{0} \eta_{t}^{x} \omega\right)-\alpha\left(V_{t}^{0} \eta_{t}^{x} \omega\right)=1$ has exactly the solutions $x_{t}^{-}$and $x_{t}^{+}$ and $\alpha_{t}, \beta_{t}$ are the restrictions to $\left[x_{t}^{-}, x_{t}^{+}\right]$of the indicator functions of $\Gamma, p, \omega$ for the point $t$. Moreover, $\Gamma$ may be chosen so that $\Gamma \backslash\{t\}$ is locally a $C^{2}$-curve and the weight $\omega$ may be chosen to be continuous on $\Gamma \backslash\{t\}$.

Thus, the conditions $\left(P_{1}\right)-\left(P_{4}\right)$ are characteristic for the indicator functions of $\Gamma, p, \omega$.

Logarithmic leaves. To understand the shape of the leaf $\mathcal{L}\left(z, w ; p, \alpha_{t}, \beta_{t}\right)$ in the case $\delta_{t}^{-}<\delta_{t}^{+}$, we introduce the notion of the logarithmic leaf. Let $1<p<\infty$, and let $\delta_{1}, \delta_{2}, \mu_{1}, \mu_{2}, \nu_{1}, \nu_{2}$ be any real numbers satisfying

$$
\delta_{1} \leq \delta_{2}, \quad 0<\mu_{1} \leq \nu_{1}<1, \quad 0<\mu_{2} \leq \nu_{2}<1 .
$$

Then

$$
\min \left\{\mu_{1}+\delta_{1} x, \mu_{2}+\delta_{2} x\right\} \leq \max \left\{\nu_{1}+\delta_{1} x, \nu_{2}+\delta_{2} x\right\}
$$

for all $x \in \mathbf{R}$. We denote by $Y^{0}\left(\delta_{1}, \delta_{2} ; \mu_{1}, \mu_{2}, \nu_{1}, \nu_{2}\right)$ the set of all $\gamma=x+i y \in \mathbf{C}$ for which

$$
\min \left\{\mu_{1}+\delta_{1} x, \mu_{2}+\delta_{2} x\right\} \leq y \leq \max \left\{\nu_{1}+\delta_{1} x, \nu_{2}+\delta_{2} x\right\} .
$$

For $z, w \in \mathbf{C}$, the logarithmic leaf $\mathcal{L}^{0}\left(z, w ; \delta_{1}, \delta_{2} ; \mu_{1}, \mu_{2}, \nu_{1}, \nu_{2}\right)$ is defined as the set

$$
\left\{M_{z, w}\left(e^{2 \pi \gamma}\right): \gamma \in Y^{0}\left(\delta_{1}, \delta_{2} ; \mu_{1}, \mu_{2}, \nu_{1}, \nu_{2}\right)\right\} \cup\{z, w\} .
$$

If $\delta_{1}=\delta_{2}=: \delta$, then $Y^{0}\left(\delta, \delta ; \mu_{1}, \mu_{2}, \nu_{1}, \nu_{2}\right)$ degenerates to the stripe of all $\gamma=$ $x+i y \in \mathbf{C}$ satisfying

$$
\min \left\{\mu_{1}, \mu_{2}\right\}+\delta x \leq y \leq \max \left\{\nu_{1}, \nu_{2}\right\}+\delta x
$$





Figure 5. Figure 5a shows the graphs of the functions in (3.11); Figure 5b shows the boundary of the corresponding logarithmic leaf.

and hence

$$
\mathcal{L}^{0}\left(z, w ; \delta, \delta ; \mu_{1}, \mu_{2}, \nu_{1}, \nu_{2}\right)=\mathcal{S}\left(z, w ; \delta ; \min \left\{\mu_{1}, \mu_{2}\right\}, \max \left\{\nu_{1}, \nu_{2}\right\}\right)
$$

is a spiralic horn for $\min \left\{\mu_{1}, \mu_{2}\right\}<\max \left\{\nu_{1}, \nu_{2}\right\}$ and a logarithmic double-spiral for $\min \left\{\mu_{1}, \mu_{2}\right\}=\max \left\{\nu_{1}, \nu_{2}\right\}$; in the latter case we necessarily have $\mu_{1}=\mu_{2}=\nu_{1}=$ $\nu_{2}$ due to $(3.10)$.

So let $\delta_{1}<\delta_{2}$ and look at Figure 5. We first of all observe that a logarithmic leaf is bounded by pieces of at most four logarithmic double-spirals. Put

$$
\alpha^{0}(x):=\min \left\{\mu_{1}+\delta_{1} x, \mu_{2}+\delta_{2} x\right\}, \beta^{0}(x):=\max \left\{\nu_{1}+\delta_{1} x, \nu_{2}+\delta_{2} x\right\} .
$$

From (3.10) we see that $\beta^{0}(0)-\alpha^{0}(0)<1$, and the condition $\delta_{1}<\delta_{2}$ implies that $\beta^{0}(x)-\alpha^{0}(x) \rightarrow+\infty$ as $x \rightarrow \pm \infty$. Thus, the equation $\beta^{0}(x)-\alpha^{0}(x)=1$ has exactly two solutions $x_{-}^{0}<0$ and $x_{+}^{0}>0$. Because

$$
\begin{aligned}
& \left\{\gamma=x+i y \in Y^{0}\left(\delta_{1}, \delta_{2} ; \mu_{1}, \mu_{2}, \nu_{1}, \nu_{2}\right): x \leq x_{-}^{0}\right\} \\
& \supset\left\{\gamma=x+i y \in \mathbf{C}: x \leq x_{-}^{0}, \alpha^{0}\left(x_{-}^{0}\right) \leq y<\beta^{0}\left(x_{-}^{0}\right)\right\}=: \Pi_{-}^{0}
\end{aligned}
$$

and

$$
\begin{aligned}
& \left\{\gamma=x+i y \in Y^{0}\left(\delta_{1}, \delta_{2} ; \mu_{1}, \mu_{2}, \nu_{1}, \nu_{2}\right): x \geq x_{+}^{0}\right\} \\
& \supset\left\{\gamma=x+i y \in \mathbf{C}: x \geq x_{+}^{0}, \alpha^{0}\left(x_{+}^{0}\right) \leq y<\beta^{0}\left(x_{+}^{0}\right)\right\}=: \Pi_{+}^{0},
\end{aligned}
$$

and because the map $\gamma \mapsto M_{z, w}\left(e^{2 \pi \gamma}\right)$ has the period $i$, it follows that this map gives a bijection between

$$
\left\{\gamma=x+i y \in Y^{0}\left(\delta_{1}, \delta_{2} ; \mu_{1}, \mu_{2}, \nu_{1}, \nu_{2}\right): x_{-}^{0}<x<x_{+}^{0}\right\} \cup \Pi_{-}^{0} \cup \Pi_{+}^{0}
$$

and the logarithmic leaf $\mathcal{L}^{0}\left(z, w ; \delta_{1}, \delta_{2} ; \mu_{1}, \mu_{2}, \nu_{1}, \nu_{2}\right)$ minus $\{z, w\}$. It is easily seen that $\Pi_{\mp}^{0}$ are mapped onto closed disks punctured at $z$ and $w$ and having the center $\left(w e^{2 \pi x_{ \pm}^{0}}-z e^{-2 \pi x_{ \pm}^{0}}\right) /\left(2 \sinh \left(2 \pi x_{ \pm}^{0}\right)\right)$ and the radius $|w-z| /\left( \pm 2 \sinh \left(2 \pi x_{ \pm}^{0}\right)\right)$, respectively. In Figure 6a the set (3.12) is represented as the union of subsets $\mathrm{A}, \mathrm{B}, \ldots, \mathrm{I}$ (the regions labeled by $\mathrm{J}$ and $\mathrm{K}$ are not contained in the set (3.12)); in Figure $6 \mathrm{~b}$ the same letters are used to indicate the images of these subsets. We remark that in Figure 6 a we have $\mathrm{A}=\Pi_{-}^{0}, \mathrm{~B}=\Pi_{+}^{0}$ and that the set $\{\gamma=x+i y \in$ 

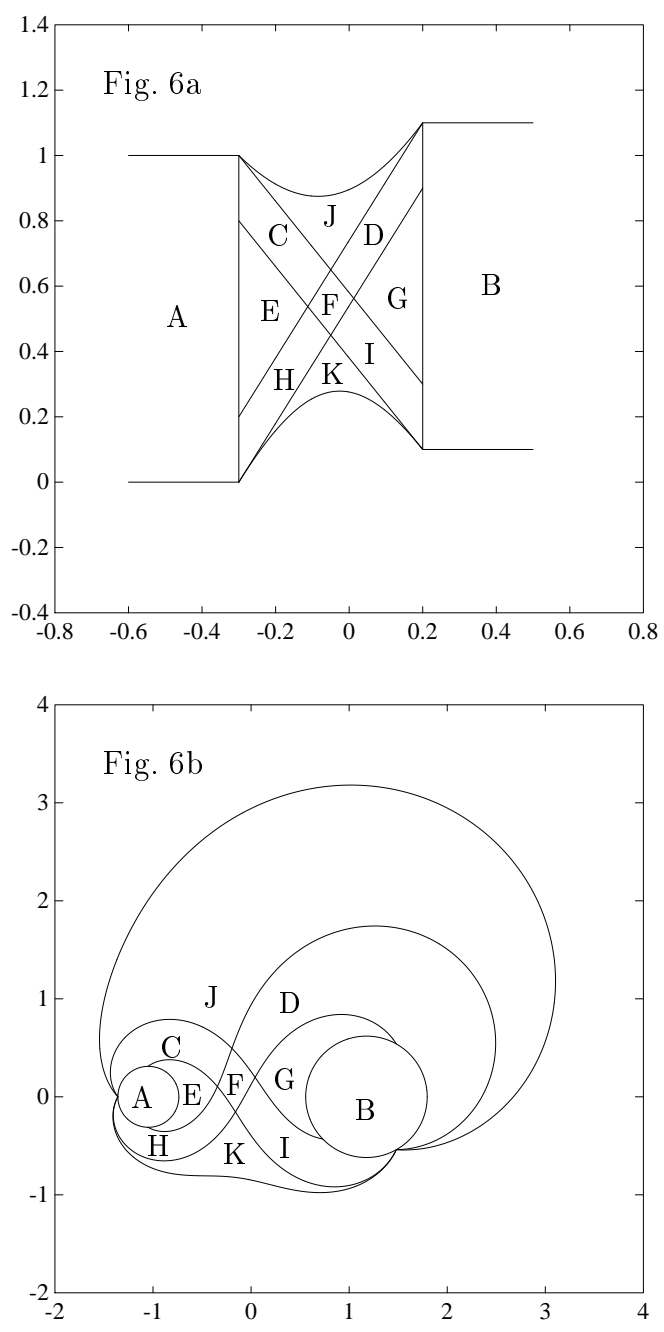

FiguRE 6

$\left.Y^{0}\left(\delta_{1}, \delta_{2} ; \mu_{1}, \mu_{2}, \nu_{1}, \nu_{2}\right): x_{-}^{0}<x<x_{+}^{0}\right\}$ is divided into the subsets C,D,E,F,G,H,I by the graphs of the functions $y=\mu_{1}+\delta_{1} x, y=\nu_{1}+\delta_{1} x, y=\mu_{2}+\delta_{2} x, y=\nu_{2}+\delta_{2} x$.

General leaves as logarithmic leaves with a halo. Let us now return to the general case and suppose $\delta_{t}^{-}<\delta_{t}^{+}$. By Theorem 3.2(d), the asymptote to $y=1 / p+\beta_{t}(x)$ as $x \rightarrow+\infty$ has the equation $y=1 / p+\nu_{t}^{+}+\delta_{t}^{+} x$. The parallel to this asymptote through the point $x_{t}^{+}+i\left(1 / p+\beta_{t}\left(x_{t}^{+}\right)\right)$has the equation $y=$ $1 / p+\beta_{t}\left(x_{t}^{+}\right)+\delta_{t}^{+}\left(x-x_{t}^{+}\right)$. Since $\beta_{t}$ is convex, it follows that

$$
\frac{1}{p}+\beta_{t}(x) \geq \frac{1}{p}+\beta_{t}\left(x_{t}^{+}\right)+\delta_{t}^{+}\left(x-x_{t}^{+}\right) \geq \frac{1}{p}+\nu_{t}^{+}+\delta_{t}^{+} x \text { for } x \in\left[x_{t}^{-}, x_{t}^{+}\right]
$$


Analogously we get

$$
\begin{aligned}
\frac{1}{p}+\beta_{t}(x) & \geq \frac{1}{p}+\beta_{t}\left(x_{t}^{-}\right)+\delta_{t}^{-}\left(x-x_{t}^{-}\right) \\
& \geq \frac{1}{p}+\nu_{t}^{-}+\delta_{t}^{-} x \text { for } x \in\left[x_{t}^{-}, x_{t}^{+}\right], \\
\frac{1}{p}+\alpha_{t}(x) & \leq \frac{1}{p}+\alpha_{t}\left(x_{t}^{-}\right)+\delta_{t}^{+}\left(x-x_{t}^{-}\right) \\
& \leq \frac{1}{p}+\mu_{t}^{+}+\delta_{t}^{+} x \text { for } x \in\left[x_{t}^{-}, x_{t}^{+}\right], \\
\frac{1}{p}+\alpha_{t}(x) & \leq \frac{1}{p}+\alpha_{t}\left(x_{t}^{+}\right)+\delta_{t}^{-}\left(x-x_{t}^{+}\right) \\
& \leq \frac{1}{p}+\mu_{t}^{-}+\delta_{t}^{-} x \text { for } x \in\left[x_{t}^{-}, x_{t}^{+}\right] .
\end{aligned}
$$

Figure 6 a shows the representation of the set

$$
\left\{x+i y \in \Pi_{t}: \frac{1}{p}+\alpha_{t}(x) \leq y \leq \frac{1}{p}+\beta_{t}(x)\right\} \cup \Pi_{t}^{-} \cup \Pi_{t}^{+}
$$

as the union of the subsets $\mathrm{A}, \mathrm{B}, \ldots, \mathrm{K}$, where $\mathrm{A}=\Pi_{t}^{-}, \mathrm{B}=\Pi_{t}^{+}$and the other subsets are obtained from the set $\left\{x+i y \in \Pi_{t}: 1 / p+\alpha_{t}(x) \leq y \leq 1 / p+\beta_{t}(x)\right\}$ by dividing it by the graphs of the functions in the middle of the estimates (3.13)-(3.16). Figure $6 \mathrm{~b}$ shows the parts of the leaf $\mathcal{L}\left(z, w ; p, \alpha_{t}, \beta_{t}\right)$ corresponding to the sets $\mathrm{A}, \mathrm{B}, \ldots, \mathrm{K}$. Thus, a leaf is a logarithmic leaf with a "halo". In Figure $6 \mathrm{~b}$ the halo is $\mathrm{J} \cup \mathrm{K}$.

Estimates for leaves via logarithmic leaves. We know everything about the leaf $\mathcal{L}\left(z, w ; p, \alpha_{t}, \beta_{t}\right)$ if only the indicator functions $\alpha_{t}$ and $\beta_{t}$ are available. As this is in general not the case, the question about estimating leaves by certain parameters is emerging.

From (3.13)-(3.16) we see that the leaf $\mathcal{L}\left(z, w ; p, \alpha_{t}, \beta_{t}\right)$ contains the logarithmic leaf

$$
\mathcal{L}^{0}\left(z, w ; \delta_{t}^{-}, \delta_{t}^{+} ; \tilde{\mu}_{t}^{-}, \tilde{\mu}_{t}^{+}, \tilde{\nu}_{t}^{-}, \tilde{\nu}_{t}^{+}\right)
$$

with

$$
\begin{aligned}
& \tilde{\mu}_{t}^{-}:=\frac{1}{p}+\alpha_{t}\left(x_{t}^{+}\right)-\delta_{t}^{-} x_{t}^{+}, \quad \tilde{\mu}_{t}^{+}:=\frac{1}{p}+\alpha_{t}\left(x_{t}^{-}\right)-\delta_{t}^{+} x_{t}^{-}, \\
& \tilde{\nu}_{t}^{-}:=\frac{1}{p}+\beta_{t}\left(x_{t}^{-}\right)-\delta_{t}^{-} x_{t}^{-}, \quad \tilde{\nu}_{t}^{+}:=\frac{1}{p}+\beta_{t}\left(x_{t}^{+}\right)-\delta_{t}^{+} x_{t}^{+},
\end{aligned}
$$

and that the logarithmic leaf (3.17) in turn contains the logarithmic leaf

$$
\mathcal{L}^{0}\left(z, w ; \delta_{t}^{-}, \delta_{t}^{+} ; \frac{1}{p}+\mu_{t}^{-}, \frac{1}{p}+\mu_{t}^{+}, \frac{1}{p}+\nu_{t}^{-}, \frac{1}{p}+\nu_{t}^{+}\right) .
$$

Thus, we may estimate the leaf $\mathcal{L}\left(z, w ; p, \alpha_{t}, \beta_{t}\right)$ from inside by (3.17) and (3.20). The estimate via (3.17) is almost the best estimate by a logarithmic leaf, but it involves the parameters (3.18), (3.19), which are only available if we can solve the equation $\beta_{t}(x)-\alpha_{t}(x)=1$. The estimate by (3.20) is more crude but only contains parameters given by Theorem $3.2(\mathrm{~d})$. 
Taking into account the convexity of $\beta_{t}$ and the concavity of $\alpha_{t}$, we get the inequalities

$$
\begin{aligned}
& \frac{1}{p}+\beta_{t}(x) \leq \max \left\{\frac{1}{p}+\beta_{t}(0)+\delta_{t}^{-} x, \frac{1}{p}+\beta_{t}(0)+\delta_{t}^{+} x\right\}, \\
& \frac{1}{p}+\alpha_{t}(x) \geq \min \left\{\frac{1}{p}+\alpha_{t}(0)+\delta_{t}^{-} x, \frac{1}{p}+\alpha_{t}(0)+\delta_{t}^{+} x\right\},
\end{aligned}
$$

which imply that the leaf $\mathcal{L}\left(z, w ; p, \alpha_{t}, \beta_{t}\right)$ is a subset of the logarithmic leaf

$$
\mathcal{L}^{0}\left(z, w ; \delta_{t}^{-}, \delta_{t}^{+} ; \frac{1}{p}+\mu_{t}, \frac{1}{p}+\mu_{t}, \frac{1}{p}+\nu_{t}, \frac{1}{p}+\nu_{t}\right)
$$

where $\mu_{t}:=\alpha_{t}(0)$ and $\nu_{t}:=\beta_{t}(0)$. This is again a crude estimate from outside by a logarithmic leaf which may be improved by invoking the solutions of the equation $\beta_{t}(x)-\alpha_{t}(x)=1$.

Indicator set. For $t \in \Gamma$ and $\gamma \in \mathbf{C}$, put

$$
\varphi_{t, \gamma}(\tau):=\left|(\tau-t)^{\gamma}\right|=|\tau-t|^{\operatorname{Re} \gamma} e^{-\operatorname{Im} \gamma \arg (\tau-t)} \quad(\tau \in \Gamma \backslash\{t\})
$$

and define

$$
N_{t}:=N_{t}(\Gamma, p, \omega):=\left\{\gamma \in \mathbf{C}: \varphi_{t, \gamma} \omega \in A_{p}(\Gamma)\right\} .
$$

We call $N_{t}$ the indicator set of $\Gamma, p, \omega$ at the point $t$. It turns out that the indicator set $N_{t}$ carries the whole information about the shape of the leaf $\mathcal{L}\left(z, w ; p, \alpha_{t}, \beta_{t}\right)$.

Theorem 3.5. If $\alpha_{t}$ and $\beta_{t}$ are given by (3.4), then

$$
N_{t}=\left\{\gamma \in \mathbf{C}:-\frac{1}{p}<\operatorname{Re} \gamma+\alpha_{t}(\operatorname{Im} \gamma) \leq \operatorname{Re} \gamma+\beta_{t}(\operatorname{Im} \gamma)<\frac{1}{q}\right\} .
$$

Notice that in the same way the values $\alpha_{t}(x)$ and $\beta_{t}(x)$ of the indicator functions for which $\beta_{t}(x)-\alpha_{t}(x)>1$ do not contribute anything to the leaf, the values $\alpha_{t}(\operatorname{Im} \gamma)$ and $\beta_{t}(\operatorname{Im} \gamma)$ do not play a role in $(3.23)$ whenever $\beta_{t}(\operatorname{Im} \gamma)-\alpha_{t}(\operatorname{Im} \gamma)>1$.

Curve and weight parameters. Suppose all we know about the indicator functions are the slopes $\delta_{t}^{-}, \delta_{t}^{+}$of their asymptotes and their values $\mu_{t}:=\alpha_{t}(0)$ and $\nu_{t}:=\beta_{t}(0)$ at the origin. Since $\mu_{t}=\alpha\left(V_{t}^{0} \omega\right)$ and $\nu_{t}=\beta\left(V_{t}^{0} \omega\right)$ and neither the exponent $p$ nor the curve $\Gamma$ explicitly occurs in $\alpha\left(V_{t}^{0} \omega\right)$ and $\beta\left(V_{t}^{0} \omega\right)$, we refer to $\mu_{t}$ and $\nu_{t}$ as weight parameters. The following theorem shows that $\delta_{t}^{-}$and $\delta_{t}^{+}$are really independent of $p$ and $\omega$ and are therefore intrinsic characteristics of the curve $\Gamma$.

Theorem 3.6. With $\eta_{t}(\tau):=e^{-\arg (\tau-t)}(\tau \in \Gamma \backslash\{t\})$, we have

$$
\delta_{t}^{-}=\alpha\left(V_{t}^{0} \eta_{t}\right), \delta_{t}^{+}=\beta\left(V_{t}^{0} \eta_{t}\right),
$$

Spirality. The curve $\Gamma$ is said to be spiralic at a point $t \in \Gamma$ if $\delta_{t}^{-}=\delta_{t}^{+}=: \delta_{t}$. For example, one can show that $\Gamma$ is spiralic at $t$ if (2.3) holds.

Let $M$ be a nonempty convex open subset of $\mathbf{C}$. For each $y \in \mathbf{R}$, let $M(y)$ denote the intersection of $M$ with the horizontal line through the point $i y$. The set $M(y)$ is an interval and we denote by $|M(y)|$ the length of this interval. The width of $M$ is defined as $\sup _{y \in \mathbf{R}}|M(y)|$. The open set between two parallel straight lines of the plane is called an open stripe. 
Theorem 3.7. If $\Gamma$ is spiralic at $t$, then the indicator functions are

$$
\alpha_{t}(x)=\mu_{t}+\delta_{t} x, \beta_{t}(x)=\nu_{t}+\delta_{t} x,
$$

the leaf $\mathcal{L}\left(z, w ; p, \alpha_{t}, \beta_{t}\right)$ is the spiralic horn

$$
\mathcal{S}\left(z, w ; \delta_{t} ; 1 / p+\mu_{t}, 1 / p+\nu_{t}\right),
$$

and the indicator set $N_{t}$ is an open stripe of width at most 1 containing the origin. Given $p \in(1, \infty)$ and any open stripe $N$ of width at most 1 which contains the origin, there exist a Carleson Jordan curve $\Gamma$ spiralic at some point $t \in \Gamma$ and a weight $\omega \in A_{p}(\Gamma)$ such that $N=N_{t}(\Gamma, p, \omega)$.

The first part of this theorem results from (3.6), (3.7), (3.23), the second part was proved in [4]. We stated this theorem mainly in order to emphasize that for spiralic curves all the information about the spectra of Toeplitz operators with piecewise continuous symbols is available from the parameters $\delta_{t}^{-}=\delta_{t}^{+}=: \delta_{t}$ and $\mu_{t}, \nu_{t}$

Powerlikeness. We say that a weight $\omega \in A_{p}(\Gamma)$ is powerlike at a point $t \in \Gamma$ if $\mu_{t}=\nu_{t}=: \lambda_{t}$. For instance, if

$$
\omega(\tau)=|\tau-t|^{\lambda} v(\tau) \quad(\tau \in \Gamma)
$$

with a weight $v \in A_{p}(\Gamma)$ which is continuous and nonzero at $t$ (and in particular, if $\omega$ is a pure power weight of the form (2.1)), then one can show that $\mu_{t}=\nu_{t}=\lambda$.

Theorem 3.8. If $\omega$ is powerlike at $t$, then the indicator functions are given by

$$
\alpha_{t}(x)=\lambda_{t}+\min \left\{\delta_{t}^{-} x, \delta_{t}^{+} x\right\}, \beta_{t}(x)=\lambda_{t}+\max \left\{\delta_{t}^{-} x, \delta_{t}^{+} x\right\},
$$

the leaf $\mathcal{L}\left(z, w ; p, \alpha_{t}, \beta_{t}\right)$ is the logarithmic leaf

$$
\mathcal{L}^{0}\left(z, w ; \delta_{t}^{-}, \delta_{t}^{+} ; \frac{1}{p}+\lambda_{t}, \frac{1}{p}+\lambda_{t}, \frac{1}{p}+\lambda_{t}, \frac{1}{p}+\lambda_{t}\right),
$$

and the indicator set $N_{t}$ is an open stripe or open parallelogram of width equal to 1 containing the origin. Given $p \in(1, \infty)$ and any open stripe or open parallelogram $N$ of width equal to 1 containing the origin, there exists a Carleson Jordan curve and a weight $\omega \in A_{p}(\Gamma)$ powerlike at some point $t \in \Gamma$ such that $N=N_{t}(\Gamma, p, \omega)$.

Note that again everything may be expressed in terms of the parameters $\delta_{t}^{-}, \delta_{t}^{+}$, and $\mu_{t}=\nu_{t}=: \lambda_{t}$. The first half of the theorem is obvious from the preceding discussion; its second half was in principle proved in [5]. Figures 3 and 7 provide examples of the logarithmic leaves (3.25).

Interference. Let $L$ be a nonempty subset of $\mathbf{C}$. We call two points $\lambda_{1}, \lambda_{2} \in L$ separated if there exists a point $s \in L$ such that $L \backslash\{s\}$ is disconnected and $\lambda_{1}, \lambda_{2}$ lie in different connected components of $L \backslash\{s\}$.

Theorem 3.9. If $\Gamma$ is spiralic at $t$, then $z$ and $w$ are boundary points of (the spiralic horn $) \mathcal{L}\left(z, w ; p, \alpha_{t}, \beta_{t}\right)$, while if $\omega$ is powerlike at $t$, then $z$ and $w$ are separated points of (the logarithmic leaf $) \mathcal{L}\left(z, w ; p, \alpha_{t}, \beta_{t}\right)$.

This is an immediate consequence of Theorems 3.7 and 3.8. We remark that the point

$$
s:=M_{z, w}\left(e^{2 \pi i\left(1 / p+\lambda_{t}\right)}\right)
$$

is always a separating point of the logarithmic leaf (3.25). 



Figure 7. The set $\left\{x+i y \in \mathbf{C}: 1 / p+\alpha_{t}(x) \leq y \leq 1 / p+\right.$ $\left.\beta_{t}(x), x_{t}^{-}<x<x_{t}^{+}\right\}$with $\alpha_{t}$ and $\beta_{t}$ as in Theorem 3.8 and the two half-stripes $\Pi_{t}^{-}$and $\Pi_{t}^{+}$are indicated in Figure $7 \mathrm{a}$. The corresponding logarithmic leaf (3.25) is shown in Figure 7b.

Thus, the appearance of logarithmic leaves in which $z$ and $w$ are inner and nonseparated and, all the more, of logarithmic leafs with a halo is caused by some kind of interference between the oscillation of the curve ("non-spirality") and the oscillation of the weight ("non-powerlikeness"). See Figures 8 and 9 for concrete examples.

Narrowness of the indicator set. We say that a subset $N$ of the plane $\mathbf{C}$ is narrow if there are two open stripes $S_{1}$ and $S_{2}$ of width at most 1 such that $N$ is contained in $\Pi:=S_{1} \cap S_{2}$ and

$$
\inf _{x+i y \in \Pi} y=\inf _{x+i y \in N} y, \quad \sup _{x+i y \in \Pi} y=\sup _{x+i y \in N} y .
$$

Clearly, open stripes of width at most 1 are narrow, and these are the only unbounded open and narrow sets. A bounded narrow set necessarily has two "peaks" at opposite vertices of the parallelogram $\Pi=S_{1} \cap S_{2}$. In particular, ellipses or any sets with smooth boundaries are never narrow.

Theorem 3.10. The indicator set $N_{t}$ is always an open, convex, narrow set containing the origin. Given $p \in(1, \infty)$ and any open, convex, narrow set $N$ containing the origin, there exist a Carleson Jordan curve $\Gamma$ and a weight $\omega \in A_{p}(\Gamma)$ such that $N$ coincides with the indicator set $N_{t}(\Gamma, p, \omega)$ for some $t \in \Gamma$.

This theorem is essentially a reformulation of Theorems 3.3 and 3.4 on the basis of Theorem 3.5.

Symbol calculus for singular integral operators. Let $\mathcal{B}$ be the Banach algebra of all bounded linear operators on $L^{p}(\Gamma, \omega)$ and let $\mathcal{K}$ stand for the ideal of the compact operators in $\mathcal{B}$. For $a \in P C(\Gamma)$, denote by $M(a) \in \mathcal{B}$ the multiplication operator $f \mapsto a f$. Finally, define $\operatorname{alg}(P C, S)$ as the smallest closed subalgebra of $\mathcal{B}$ containing $\{M(a): a \in P C(\Gamma)\}$ and the Cauchy singular integral operator $S$. We put

$$
M:=M_{\Gamma, p, \omega}:=\bigcup_{t \in \Gamma}\left(\{t\} \times \mathcal{L}\left(0,1 ; p, \alpha_{t}, \beta_{t}\right)\right)
$$



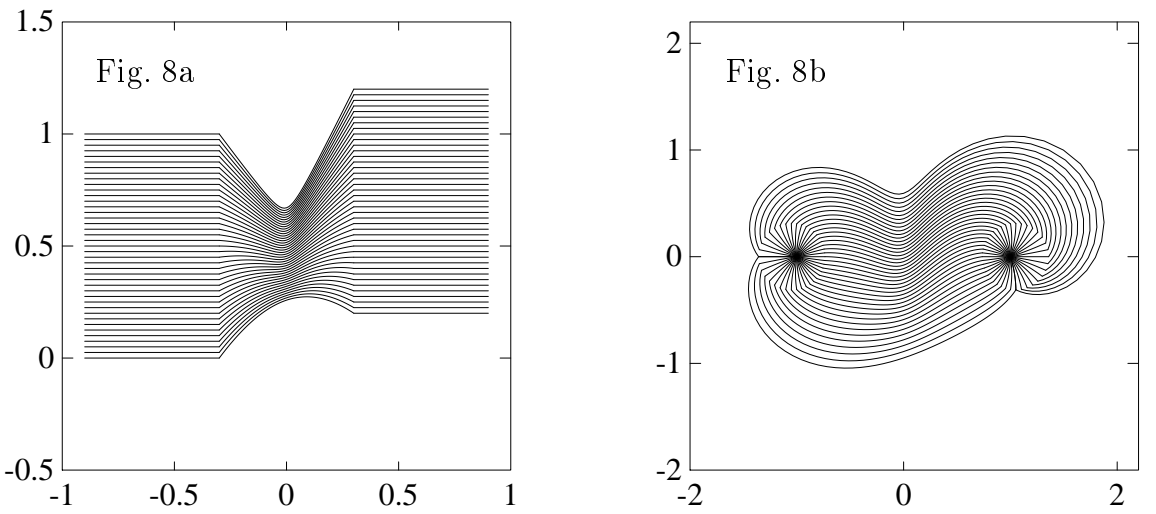

Figure 8 . Figures $8 \mathrm{a}$ and $8 \mathrm{~b}$ show us one more way to understand Figures 4 and 6 . It is clearly seen that the leaf in Figure $8 \mathrm{~b}$ has no separating points and that the points -1 and 1 are inner points.

and call $M$ the "leaf bundle" of $\Gamma, p, \omega$.

Theorem 3.11. (a) $\mathcal{K}$ is a subset of the algebra $\operatorname{alg}(P C, S)$, and the quotient algebra $\operatorname{alg}(P C, S) / \mathcal{K}$ is inverse closed in the quotient algebra $\mathcal{B} / \mathcal{K}$.

(b) For each point $(t, \lambda) \in M$ the map

$$
\operatorname{Sym}_{t, \lambda}:\{M(a): a \in P C(\Gamma)\} \cup\{S\} \rightarrow \mathbf{C}^{2 \times 2}
$$

given by

$$
\begin{gathered}
\operatorname{Sym}_{t, \lambda}(M(a))=\left(\begin{array}{cc}
a(t+0) & 0 \\
0 & a(t-0)
\end{array}\right), \\
\operatorname{Sym}_{t, \lambda}(S)=\left(\begin{array}{cc}
2 \lambda-1 & 2 \sqrt{\lambda(1-\lambda)} \\
2 \sqrt{\lambda(1-\lambda)} & 1-2 \lambda
\end{array}\right)
\end{gathered}
$$

extends to a Banach algebra homomorphism

$$
\operatorname{Sym}_{t, \lambda}: \operatorname{alg}(P C, S) \rightarrow \mathbf{C}^{2 \times 2}
$$

with the property that $\operatorname{Sym}_{t, \lambda}(K)$ is the zero matrix for every compact operator $K$.

(c) An operator $A \in \operatorname{alg}(P C, S)$ is Fredholm on the space $L^{p}(\Gamma, \omega)$ if and only if $\operatorname{det} \operatorname{Sym}_{t, \lambda}(A) \neq 0$ for all $(t, \lambda) \in M$.

In (b), we understand by $\sqrt{\lambda(1-\lambda)}$ any function $f: \mathbf{C} \rightarrow \mathbf{C}$ such that $(f(\lambda))^{2}=$ $\lambda(1-\lambda)$ for all $\lambda \in \mathbf{C}$. Notice that on the leaf $\mathcal{L}\left(0,1 ; p, \alpha_{t}, \beta_{t}\right)$ there is in general no continuous branch of $\sqrt{\lambda(1-\lambda)}$.

For piecewise smooth curves $\Gamma$, this theorem is Gohberg and Krupnik's [19] in case $\omega$ is a power weight of the form (2.1), and it was established in [16] and [21] in the case where $\omega$ is an arbitrary weight in $A_{p}(\Gamma)$. For Carleson curves satisfying (2.3) at each point and weights $\omega \in A_{p}(\Gamma)$ as well as for general Carleson curves and the weight $\omega \equiv 1$, the theorem was proved in [4] and [5]. We remark that once Theorem 3.1 is available, Theorem 3.11 can be proved by employing local principles in conjunction with the "two projections theorem" of Finck, Roch, Silbermann [16] or the "extension theorem" of Gohberg and Krupnik [21]. 

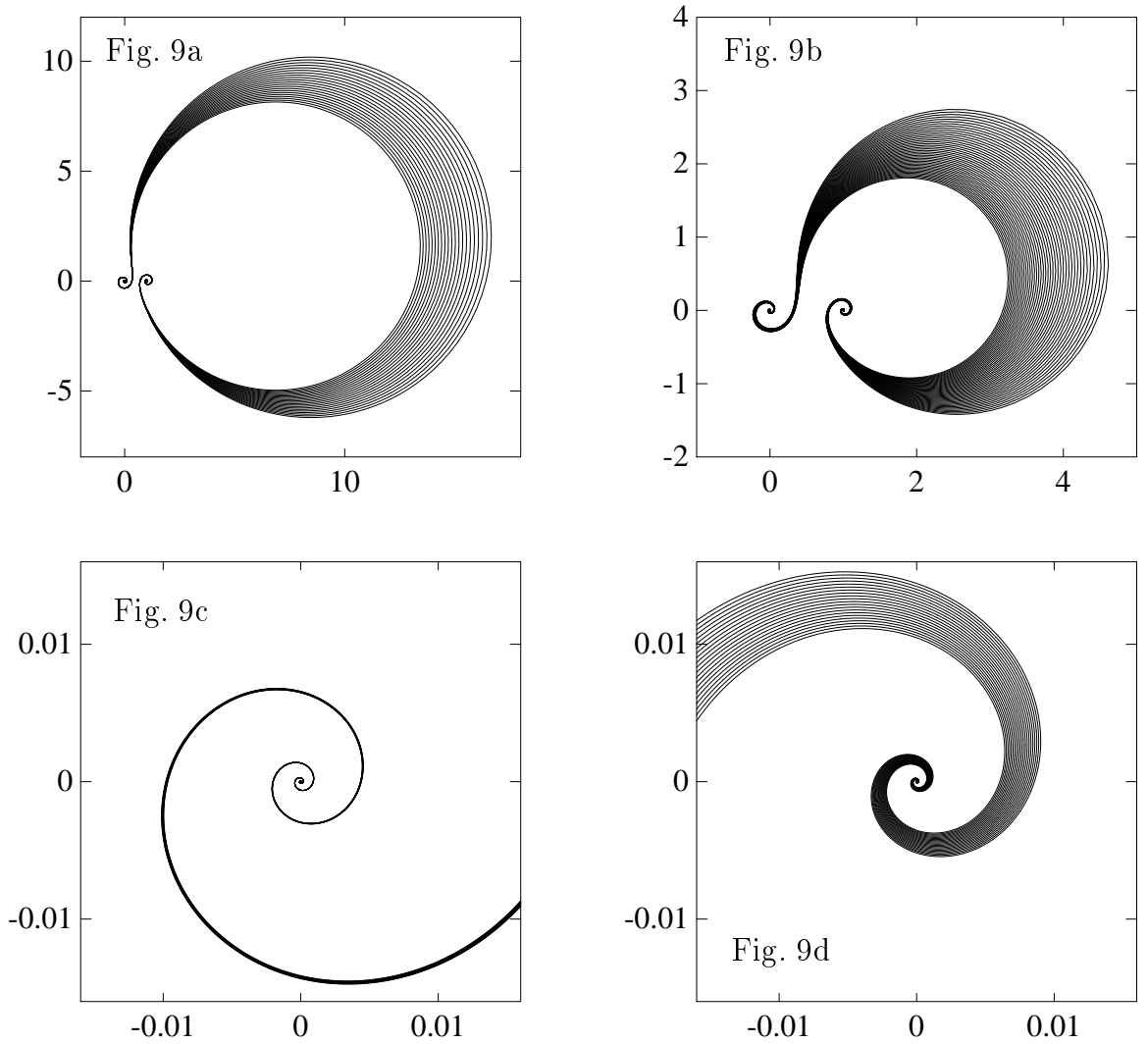

Figure 9. These pictures nicely illustrate the beauty of leaves. Figure 9a shows a spiralic horn; in Figure 9b we plotted a leaf emerging when choosing $\alpha_{t}$ and $\beta_{t}$ as hyperbolas, and thus a leaf containing a halo. Consequently, the boundary of the set in Figure 9a consists of two logarithmic double-spirals, while (though this is hardly visible in the case at hand) no piece of the boundary of the leaf in Figure 9b is a piece of some logarithmic double-spiral. When looking at Figure 9a and Figure 9b with a magnifying glass, which is done in Figure $9 \mathrm{c}$ ind Figure 9d, we really see that the points 0 and 1 belong to the boundary of the leaf in Figure 9 a but are inner points of the leaf in Figure $9 \mathrm{~b}$.

\section{Preliminaries to the proofs}

The case of a general symbol $a \in P C(\Gamma)$ may be reduced to the consideration of certain "canonical" symbols by means of localization techniques. Fix $t \in \Gamma$. Without loss of generality assume that the origin is contained in the interior $D_{+}$of $\Gamma$. The interior and exterior of the complex unit circle can be conformally mapped onto the interior $D_{+}$and the exterior $D_{-}$of $\Gamma$, respectively, so that the point 1 is mapped to $t$ and the point $0 \in D_{+}$and $\infty \in D_{-}$remain fixed. Let $\Lambda_{0}$ and $\Lambda_{\infty}$ denote the images of $[0,1]$ and $[1, \infty) \cup\{\infty\}$ under this map. The curve $\Lambda_{0} \cup \Lambda_{\infty}$ joins 0 to $\infty$ and meets $\Gamma$ at exactly one point, namely $t$. Let $\arg z$ be a continuous 
branch of the argument in $\mathbf{C} \backslash\left(\Lambda_{0} \cup \Lambda_{\infty}\right)$. For $\gamma \in \mathbf{C}$, define

$$
z^{\gamma}:=|z|^{\gamma} e^{i \gamma \arg z} \quad\left(z \in \mathbf{C} \backslash\left(\Lambda_{0} \cup \Lambda_{\infty}\right)\right) .
$$

Then, $z^{\gamma}$ is an analytic function in $\mathbf{C} \backslash\left(\Lambda_{0} \cup \Lambda_{\infty}\right)$. The restriction of $z^{\gamma}$ to $\Gamma \backslash\{t\}$ will be denoted by $g_{t, \gamma}$. Clearly, $g_{t, \gamma}$ is continuous and nonzero on $\Gamma \backslash\{t\}, g_{t, \gamma} \in P C(\Gamma)$, and

$$
g_{t, \gamma}(t+0) / g_{t, \gamma}(t-0)=e^{-2 \pi i \gamma} .
$$

Now consider the operator $T(a)-\lambda I=T(a-\lambda)$. It is well known that $a(t \pm 0)-$ $\lambda \neq 0$ for all $t \in \Gamma$ if $T(a-\lambda)$ is Fredholm (see [30] or [4]). Suppose $a(t \pm 0)-\lambda \neq 0$, choose any continuous argument of $(a(t-0)-\lambda) /(a(t+0)-\lambda)$, and define $\gamma:=$ $\gamma(t, \lambda) \in \mathbf{C}$ by

$$
\operatorname{Re} \gamma=\frac{1}{2 \pi} \arg \frac{a(t-0)-\lambda}{a(t+0)-\lambda}, \operatorname{Im} \gamma=-\frac{1}{2 \pi} \log \left|\frac{a(t-0)-\lambda}{a(t+0)-\lambda}\right| .
$$

Then $e^{-2 \pi i \gamma}=(a(t+0)-\lambda) /(a(t-0)-\lambda)$ and consequently,

$$
g_{t, \gamma}(t+0)=\mu(a(t+0)-\lambda), g_{t, \gamma}(t-0)=\mu(a(t-0)-\lambda)
$$

with some nonzero $\mu \in \mathbf{C}$.

Theorem 4.1. The operator $T(a)-\lambda I$ is Fredholm if and only if $\lambda \notin \mathcal{R}(a)$ and $T\left(g_{t, \gamma(t, \lambda)}\right)$ is Fredholm for every $t \in \Gamma$.

This theorem follows from (4.2) and the local principles exhibited in [20], [6], [4]. Notice that $\lambda \notin \mathcal{R}(a)$ if and only if $a(t \pm 0)-\lambda \neq 0$ for all $t \in \Gamma$.

In order to study Fredholmness of the "local representative" $T\left(g_{t, \gamma}\right)$, we have recourse to the following theorem by Simonenko [29], [30], [31] (also see [20], [6], [4]).

Theorem 4.2. Let $g \in L^{\infty}(\Gamma)$. Then $T(g)$ is Fredholm on $H^{p}(\Gamma, \omega)$ if and only if $g^{-1} \in L^{\infty}(\Gamma)$ and $g$ can be factored in the form

$$
g(\tau)=g_{-}(\tau) \tau^{\varkappa} g_{+}(\tau) \text { a.e. on } \Gamma
$$

where $\varkappa$ is an integer, $\varkappa \in \mathbf{Z}$, and the functions $g_{ \pm}$have the following properties:

(i) $g_{-} \in H_{-}^{p}(\Gamma, \omega), g_{-}^{-1} \in H_{-}^{q}\left(\Gamma, \omega^{-1}\right), g_{+} \in H^{q}\left(\Gamma, \omega^{-1}\right), g_{+}^{-1} \in H^{p}(\Gamma, \omega)$;

(ii) $\left|g_{+}^{-1}\right| \omega \in A_{p}(\Gamma)$.

In that case $\operatorname{Ind} T(g)=-\varkappa$.

The specially constructed functions $g_{t, \gamma}$ admit the factorization

$$
g_{t, \gamma}(\tau)=(1-t / \tau)^{\varkappa-\gamma} \tau^{\varkappa}(\tau-t)^{\gamma-\varkappa} \quad(\tau \in \Gamma \backslash\{t\})
$$

with appropriate branches of $(1-t / \tau)^{\varkappa-\gamma}$ and $(\tau-t)^{\gamma-\varkappa}$ : define $\arg z$ for $z \in$ $\mathbf{C} \backslash\left(\Lambda_{0} \cup \Lambda_{\infty}\right)$ as above, take any continuous branch of $\arg (z-t)$ for $z \in \mathbf{C} \backslash \Lambda_{\infty}$, define

$$
\arg (1-t / z)=\arg ((z-t) / z):=\arg (z-t)-\arg z
$$

for $z \in \mathbf{C} \backslash\left(\Lambda_{0} \cup \Lambda_{\infty}\right)$, and then put

$$
(z-t)^{\gamma}:=|z-t|^{\gamma} e^{i \gamma \arg (z-t)},(1-t / z)^{\gamma}:=|1-t / z|^{\gamma} e^{i \gamma \arg (1-t / z)}
$$

for $\gamma \in \mathbf{C}$. Then $\arg (1-t / z)$ can be continuously continued across $\Lambda_{\infty}$ and hence $(1-t / z)^{\gamma}$ is well-defined for all $z \in \mathbf{C} \backslash \Lambda_{0}$. It turns out the functions $(z-t)^{\gamma}$ and 
$(1-t / z)^{\gamma}$ are analytic and nonzero in $\mathbf{C} \backslash \Lambda_{\infty}$ and $\mathbf{C} \backslash \Lambda_{0}$, respectively, and are continuous on $D_{+} \cup(\Gamma \backslash\{t\})$ and $D_{-} \cup(\Gamma \backslash\{t\})$, respectively.

Recall the definition (3.22) of the indicator set. Our aim is to construct continuous functions $\alpha_{t}, \beta_{t}: \mathbf{R} \rightarrow \mathbf{R}$ satisfying

$$
\alpha_{t}(x) \leq \beta_{t}(x) \text { for all } x \in \mathbf{R}
$$

such that

$$
N_{t}=\left\{\gamma \in \mathbf{C}:-\frac{1}{p}<\operatorname{Re} \gamma+\alpha_{t}(\operatorname{Im} \gamma) \leq \operatorname{Re} \gamma+\beta_{t}(\operatorname{Im} \gamma)<\frac{1}{q}\right\} .
$$

Theorem 4.3. Suppose (4.5) and (4.6) hold for certain functions $\alpha_{t}, \beta_{t}$. Then $T\left(g_{t, \gamma}\right)$ is Fredholm on $H^{p}(\Gamma, \omega)$ if and only if

$$
\frac{1}{p}-\operatorname{Re} \gamma+\theta \alpha_{t}(-\operatorname{Im} \gamma)+(1-\theta) \beta_{t}(-\operatorname{Im} \gamma) \notin \mathbf{Z}
$$

for all $\theta \in[0,1]$.

With only minor and obvious modifications, the proof of this theorem is the proof of Theorem 8.2 of [5]. We merely remark that the idea of the proof (which goes back to Spitkovsky [33]) is as follows. A suitable choice of the integer $\varkappa$ guarantees that (4.4) is a factorization of the form (4.3) satisfying condition (i) of Theorem 4.2. Condition (ii) of that theorem is satisfied in the case at hand if and only if $\left|a_{+}^{-1}(\tau)\right| \omega(\tau)=\left|(\tau-t)^{\varkappa-\gamma}\right| \omega(\tau)$ is a weight in $A_{p}(\Gamma)$, which is the same as requiring that $\varkappa-\gamma$ be in $N_{t}$, i.e.

$$
-\frac{1}{p}<\varkappa-\operatorname{Re} \gamma+\alpha_{t}(-\operatorname{Im} \gamma) \leq \varkappa-\operatorname{Re} \gamma+\beta_{t}(-\operatorname{Im} \gamma)<\frac{1}{q},
$$

or equivalently,

$$
-\varkappa<\frac{1}{p}-\operatorname{Re} \gamma+\theta \alpha_{t}(-\operatorname{Im} \gamma)+(1-\theta) \beta_{t}(-\operatorname{Im} \gamma)<-\varkappa+1
$$

for all $\theta \in[0,1]$.

Theorem 4.4. Suppose (4.6) holds with certain functions subject to (4.5). Then for every $a \in P C(\Gamma)$ the essential spectrum of $T(a)$ on $H^{p}(\Gamma, \omega)$ is given by (3.2).

Proof. Let $\lambda \notin \mathcal{R}(a)$, i.e. $a(t \pm 0)-\lambda \neq 0$ for $t \in \Gamma$. Taking into account (4.1) we deduce from Theorem 4.3 that $T\left(g_{t, \gamma(t, \lambda)}\right)$ is not Fredholm if and only if

$$
\zeta:=(a(t-0)-\lambda) /(a(t+0)-\lambda)
$$

belongs to the set $\mathcal{L}_{p, \alpha_{t}, \beta_{t}}$ defined as

$$
\bigcup_{\theta \in[0,1]}\left\{\zeta \in \mathbf{C}: \frac{1}{p}-\frac{1}{2 \pi} \arg \zeta+\theta \alpha_{t}\left(\frac{1}{2 \pi} \log |\zeta|\right)+(1-\theta) \beta_{t}\left(\frac{1}{2 \pi} \log |\zeta|\right) \in \mathbf{Z}\right\},
$$

i.e. if and only if $\lambda \in M_{a(t-0), a(t+0)}\left(\mathcal{L}_{p, \alpha_{t}, \beta_{t}}\right)$. Write $\zeta=e^{2 \pi x} e^{2 \pi i y}$ with $x$ and $y$ in R. Then $\zeta \in \mathcal{L}_{p, \alpha_{t}, \beta_{t}}$ if and only if

$$
1 / p-y+\theta \alpha_{t}(x)+(1-\theta) \beta_{t}(x) \in \mathbf{Z} \text { for some } \theta \in[0,1],
$$

i.e. if and only if

$$
1 / p+\alpha_{t}(x) \leq y+\varkappa \leq 1 / p+\beta_{t}(x)
$$


for some $\varkappa \in \mathbf{Z}$. Consequently,

$$
\begin{aligned}
\operatorname{sp}_{\text {ess }} T\left(g_{t, \gamma(t, \lambda)}\right) & =\left\{M_{a(t-0), a(t+0)}\left(e^{2 \pi(x+i y)}\right): x+i y \in Y\left(p, \alpha_{t}, \beta_{t}\right)\right\} \cup\{a(t \pm 0)\} \\
& =\mathcal{L}\left(a(t-0), a(t+0) ; p, \alpha_{t}, \beta_{t}\right) .
\end{aligned}
$$

Formula (3.2) now follows from Theorem 4.1.

Thus, everything comes down to finding functions $\alpha_{t}, \beta_{t}$ satisfying (4.5) and (4.6), that is, to determining the indicator functions, or equivalently, to describing the indicator set $N_{t}$. To identify $N_{t}$, we have to check whether the function $\varphi_{t, \gamma} \omega$ given by

$$
\varphi_{t, \gamma}(\tau) \omega(\tau)=\left|(\tau-t)^{\gamma}\right| \omega(\tau) \quad(\tau \in \Gamma \backslash\{t\})
$$

satisfies the Muckenhoupt condition (1.2). Our strategy for accomplishing this is as follows. We associate a function $U_{t} \varphi_{t, \gamma} \omega:=U_{t}\left(\varphi_{t, \gamma} \omega\right):(0, \infty) \rightarrow(0, \infty)$ with $\varphi_{t, \gamma} \omega$ which will be shown to be submultiplicative and whose behavior near 0 and $\infty$ may therefore be characterized by two so-called indices $\alpha\left(U_{t} \varphi_{t, \gamma} \omega\right)$ and $\beta\left(U_{t} \varphi_{t, \gamma} \omega\right)$. We then prove that $\varphi_{t, \gamma} \omega \in A_{p}(\Gamma)$ if and only if

$$
-1 / p<\alpha\left(U_{t} \varphi_{t, \gamma} \omega\right) \leq \beta\left(U_{t} \varphi_{t, \gamma} \omega\right)<1 / q,
$$

and we will be able to do the "separation"

$$
\alpha\left(U_{t} \varphi_{t, \gamma} \omega\right)=\operatorname{Re} \gamma+\alpha_{t}{ }^{*}(\operatorname{Im} \gamma), \beta\left(U_{t} \varphi_{t, \gamma} \omega\right)=\operatorname{Re} \gamma+\beta_{t}{ }^{*}(\operatorname{Im} \gamma)
$$

with certain functions $\alpha_{t}{ }^{*}, \beta_{t}{ }^{*}$. By Theorem 4.4, at this point we have Theorems 3.1 and 3.5 with $\alpha_{t}{ }^{*}, \beta_{t}{ }^{*}$ in place of $\alpha_{t}, \beta_{t}$.

Unfortunately, the functions $\alpha_{t}{ }^{*}, \beta_{t}{ }^{*}$ are very complicated and such a property as the one of Theorem 3.2(d) cannot be immediately proved for $\alpha_{t}{ }^{*}, \beta_{t}{ }^{*}$. We therefore associate another submultiplicative function $V_{t}^{0} \varphi_{t, \gamma} \omega:(0, \infty) \rightarrow(0, \infty)$ with $\varphi_{t, \gamma} \omega$. The indices $\alpha\left(V_{t}^{0} \varphi_{t, \gamma} \omega\right)$ and $\beta\left(V_{t}^{0} \varphi_{t, \gamma} \omega\right)$ can again be "separated",

$$
\alpha\left(V_{t}^{0} \varphi_{t, \gamma} \omega\right)=\operatorname{Re} \gamma+\alpha_{t}(\operatorname{Im} \gamma), \beta\left(V_{t}^{0} \varphi_{t, \gamma} \omega\right)=\operatorname{Re} \gamma+\beta_{t}(\operatorname{Im} \gamma),
$$

and we can show that $\alpha_{t}, \beta_{t}$ have all the properties listed in Theorem 3.2. However, we have not been able to show in a direct way that in (4.7) the $U_{t}$ may be replaced by $V_{t}^{0}$. This will be verified indirectly.

Finally, in order to prove Theorem 3.4 we have to do some concrete computations, and for this purpose even $V_{t}^{0}$ is too unwieldy. For this reason we consider a third submultiplicative function $W_{t} \varphi_{t, \gamma} \omega$ and its indices $\alpha\left(W_{t} \varphi_{t, \gamma} \omega\right)$ and $\beta\left(W_{t} \varphi_{t, \gamma} \omega\right)$.

The rest of the paper is organized as follows. In Section 5 we introduce six transformations $U_{t}, U_{t}^{0}, V_{t}, V_{t}^{0}, W_{t}, W_{t}^{0}$ which send weights $\psi: \Gamma \rightarrow[0, \infty]$ to submultiplicative functions on $(0, \infty)$. Each of these transformations has its peculiarities. The transformation $X^{0}$ differs from the transformation $X$ merely in that a "sup" is replaced by "limsup". As a rule, when proving something, it will be more convenient to work with "sup", while for computing something, a "limsup" is preferable. In Section 6 we relate the Muckenhoupt condition for a weight $\psi: \Gamma \rightarrow[0, \infty]$ with the indices of the submultiplicative functions we have associated with $\psi$. Beginning with Section 7 , we study the case where $\psi=\varphi_{t, \gamma} \omega$. Section 7 closes with describing the indicator set $N_{t}$ in terms of $\alpha_{t}{ }^{*}, \beta_{t}{ }^{*}$, while Section 8 is devoted to proving that $\alpha_{t}{ }^{*}, \beta_{t}{ }^{*}$ and $\alpha_{t}, \beta_{t}$ coincide on $\left[x_{t}^{-}, x_{t}^{+}\right]$, which results in the desired description of the indicator set $N_{t}$ via the indicator functions $\alpha_{t}, \beta_{t}$ and thus gives Theorem 3.1 and 3.5. Section 9 contains the proof of Theorem 3.2 and 3.3; in Section 10 we prove Theorem 3.4 . 
As already pointed out in Section 3, Theorems 3.7-3.10 are more or less immediate corollaries of Theorems 3.1-3.5. Theorem 3.6 follows from Lemmas 7.1 and 9.2, and Theorem 3.11 may be proved as its special versions contained in [4], [5].

\section{Submultiplicative functions associated With Weights}

We call a function $\varrho:(0, \infty) \rightarrow[0, \infty]$ regular if it is bounded and bounded away from zero in some open neighborhood of the point 1. Equivalently, $\varrho$ is regular if and only if $\log \varrho$ is bounded near 1 . A function $\varrho:(0, \infty) \rightarrow(0, \infty]$ is said to be submultiplicative if

$$
\varrho\left(x_{1}, x_{2}\right) \leq \varrho\left(x_{1}\right) \varrho\left(x_{2}\right) \text { for all } x_{1}, x_{2} \in(0, \infty) .
$$

Clearly, a regular submultiplicative function is bounded and bounded away from zero on every segment $[a, b] \subset(0, \infty)$ and thus finite on all of $(0, \infty)$.

Theorem 5.1. If $\varrho:(0, \infty) \rightarrow(0, \infty)$ is a regular submultiplicative function, then

(a) the limits

$$
\alpha(\varrho):=\lim _{x \rightarrow 0} \frac{\log \varrho(x)}{\log x}, \beta(\varrho):=\lim _{x \rightarrow \infty} \frac{\log \varrho(x)}{\log x}
$$

exist and $-\infty<\alpha(\varrho) \leq \beta(\varrho)<+\infty$;

(b) $\varrho(x) \geq x^{\alpha(\varrho)}$ for $x \in(0,1), \varrho(x) \geq x^{\beta(\varrho)}$ for $x \in(1, \infty)$;

(c) given $\varepsilon>0$, there exists an $x_{0}=x_{0}(\varepsilon) \in(0,1)$ such that $\varrho(x) \leq x^{\alpha(\varrho)-\varepsilon}$ for $x \in\left(0, x_{0}\right)$ and $\varrho(x) \leq x^{\beta(\varrho)+\varepsilon}$ for $x \in\left(x_{0}^{-1}, \infty\right)$.

Proof. Theorem 7.6.2 of [23] and Theorem 1.3 of Chapter 2 of [25].

The numbers $\alpha(\varrho)$ and $\beta(\varrho)$ are called the lower and upper indices of $\varrho$.

Let $\Gamma$ be a Carleson Jordan curve and fix $t \in \Gamma$. For $0 \leq R_{1}<R_{2} \leq d_{t}:=$ $\max _{\tau \in \Gamma}|\tau-t|$, we define

$$
\Gamma\left(t, R_{1}, R_{2}\right):=\left\{\tau \in \Gamma: R_{1} \leq|\tau-t|<R_{2}\right\} .
$$

Thus $\Gamma(t, 0, R)$ is the portion $\Gamma(t, R)$ of $\Gamma$ in the disk $\{z \in \mathbf{C}:|z-t|<R\}$ and if $R_{1}>0$, then $\Gamma\left(t, R_{1}, R_{2}\right)$ is the portion of $\Gamma$ in the annulus $\left\{z \in \mathbf{C}: R_{1} \leq|z-t|<\right.$ $\left.R_{2}\right\}$. For a function $f$ which is integrable on $\Gamma\left(t, R_{1}, R_{2}\right)$ we put

$$
\Delta_{t}\left(f, R_{1}, R_{2}\right):=\frac{1}{\left|\Gamma\left(t, R_{1}, R_{2}\right)\right|} \int_{\Gamma\left(t, R_{1}, R_{2}\right)} f(\tau)|d \tau| .
$$

Now let $\psi: \Gamma \rightarrow[0, \infty]$ be a weight. In dependence on some additional properties of $\psi$, we define six functions

$$
U_{t} \psi, V_{t} \psi, W_{t} \psi, U_{t}^{0} \psi, V_{t}^{0} \psi, W_{t}^{0} \psi:(0, \infty) \rightarrow[0, \infty] .
$$

Suppose first that $\psi \in L_{\mathrm{loc}}^{p}(\Gamma \backslash\{t\})$ and $\psi^{-1} \in L_{\mathrm{loc}}^{q}(\Gamma \backslash\{t\})$. Fix any number $\kappa \in(0,1)$ and put

$$
\left(U_{t} \psi\right)(x)=\left\{\begin{array}{l}
\sup _{R>0}\left(\left(\Delta_{t}\left(\psi^{p}, \kappa x R, x R\right)\right)^{1 / p}\left(\Delta_{t}\left(\psi^{-q}, \kappa R, R\right)\right)^{1 / q}\right) \text { for } x \in(0,1], \\
\sup _{R>0}\left(\left(\Delta_{t}\left(\psi^{p}, \kappa R, R\right)\right)^{1 / p}\left(\Delta_{t}\left(\psi^{-q}, \kappa x^{-1} R, x^{-1} R\right)\right)^{1 / q}\right) \text { for } x \in[1, \infty),
\end{array}\right.
$$


where here and in the following $\sup _{R>0}$ means $\sup _{0<R \leq d_{t}}$. The dependence of $U_{t} \psi$ on $\kappa$ will be suppressed. Define $U_{t}^{0} \psi$ by (5.1) with $\sup _{R>0}$ replaced by $\lim \sup _{R \rightarrow 0}$.

If $\log \psi \in L^{1}(\Gamma)$, we put

$$
\left(V_{t} \psi\right)(x)=\left\{\begin{array}{l}
\sup _{R>0}\left(\exp \left(\Delta_{t}(\log \psi, 0, x R)\right) / \exp \left(\Delta_{t}(\log \psi, 0, R)\right)\right) \text { for } x \in(0,1], \\
\sup _{R>0}\left(\exp \left(\Delta_{t}(\log \psi, 0, R)\right) / \exp \left(\Delta_{t}\left(\log \psi, 0, x^{-1} R\right)\right)\right) \text { for } x \in[1, \infty),
\end{array}\right.
$$

and we denote by $V_{t}^{0} \psi$ the function given by (5.2) with $\lim \sup _{R \rightarrow 0}$ in place of $\sup _{R>0}$.

Finally, if $\psi$ is continuous on $\Gamma \backslash\{t\}$ and $\psi(\tau) \neq 0$ for all $\tau \in \Gamma \backslash\{t\}$, we define

$$
\left(W_{t} \psi\right)(x)=\left\{\begin{array}{l}
\sup _{R>0}\left(\max _{|\tau-t|=x R} \psi(\tau) / \min _{|\tau-t|=R} \psi(\tau)\right) \text { for } x \in(0,1], \\
\sup _{R>0}\left(\max _{|\tau-t|=R} \psi(\tau) / \min _{|\tau-t|=x^{-1} R} \psi(\tau)\right) \text { for } x \in[1, \infty),
\end{array}\right.
$$

and the function given by (5.3) after replacing $\sup _{R>0}$ with $\lim \sup _{R \rightarrow 0}$ is denoted by $W_{t}^{0} \psi$. In (5.3) and in what follows we use the abbreviation

$$
\max _{|\tau-t|=x R}:=\max _{\tau \in \Gamma,|\tau-t|=x R}, \quad \min _{|\tau-t|=x R}:=\min _{\tau \in \Gamma,|\tau-t|=x R} .
$$

Lemma 5.2. If $\psi: \Gamma \rightarrow[0, \infty]$ is a weight, $\psi \in L^{p}(\Gamma)$ and $\psi^{-1} \in L^{q}(\Gamma)$, then $\log \psi \in L^{1}(\Gamma)$.

Proof. Put $\Gamma^{+}:=\{\tau \in \Gamma: \psi(\tau) \in[1, \infty)\}, \Gamma^{-}:=\{\tau \in \Gamma: \psi(\tau) \in(0,1)\}$. Then

$$
|\log \psi(\tau)|<\psi(\tau) \text { for } \tau \in \Gamma^{+},|\log \psi(\tau)|<\psi^{-1}(\tau) \text { for } \tau \in \Gamma^{-}
$$

and hence,

$$
\begin{aligned}
& \int_{\Gamma}|\log \psi(\tau)||d \tau|=\int_{\Gamma^{+}}|\log \psi(\tau)||d \tau|+\int_{\Gamma^{-}}|\log \psi(\tau)||d \tau| \\
& <\int_{\Gamma^{+}} \psi(\tau)|d \tau|+\int_{\Gamma^{-}} \psi^{-1}(\tau)|d \tau| \leq \int_{\Gamma} \psi(\tau)|d \tau|+\int_{\Gamma} \psi^{-1}(\tau)|d \tau| \\
& \leq|\Gamma|^{1 / q}\left(\int_{\Gamma} \psi^{p}(\tau)|d \tau|\right)^{1 / p}+|\Gamma|^{1 / p}\left(\int_{\Gamma} \psi^{-q}(\tau)|d \tau|\right)^{1 / q}<\infty .
\end{aligned}
$$

Thus, if $\psi \in L^{p}(\Gamma)$ and $\psi^{-1} \in L^{q}(\Gamma)$, then the four functions $U_{t} \psi, U_{t}^{0} \psi, V_{t} \psi, V_{t}^{0} \psi$ are well-defined. Since $\psi \in L^{p}(\Gamma)$ and $\psi^{-1} \in L^{q}(\Gamma)$ whenever $\psi \in A_{p}(\Gamma)$, it follows that these four functions are well-defined for every weight in $A_{p}(\Gamma)$.

Actually much more can be said about the logarithm of a Muckenhoupt weight. A locally integrable function $f: \Gamma \rightarrow[-\infty, \infty]$ is said to have bounded mean oscillation, $f \in B M O(\Gamma)$, if

$$
\|f\|_{*}:=\sup _{t \in \Gamma} \sup _{R>0} \frac{1}{|\Gamma(t, R)|} \int_{\Gamma(t, R)}\left|f(\tau)-\Delta_{t}(f, 0, R)\right||d \tau|<\infty
$$


where, by the notation introduced above,

$$
\Delta_{t}(f, 0, R):=\frac{1}{|\Gamma(t ; R)|} \int_{\Gamma(t, R)} f(\tau)|d \tau|
$$

We remark that $\|\cdot\|_{*}$ is a semi-norm on $B M O(\Gamma)$ with the property that $\|f\|_{*}=0$ if and only if $f$ is a constant (a.e.). Clearly, $B M O(\Gamma) \subset L^{1}(\Gamma)$. For "nice" curves both the following lemma and its proof are well-known (see e.g. [17], Chap. VI, Sec. 6).

Lemma 5.3. If $\psi \in A_{p}(\Gamma)(1<p<\infty)$, then $\log \psi \in B M O(\Gamma)$.

Proof. We abbreviate $\Delta_{t}(\log \psi, 0, R)$ to $\Delta_{t}(R)$. The Muckenhoupt condition (1.2) for $\psi$ is equivalent to the condition

$$
\sup \left(\frac{\exp \left(-p \Delta_{t}(R)\right)}{|\Gamma(t, R)|} \int_{\Gamma(t, R)} \psi^{p}(\tau)|d \tau|\right)^{1 / p}\left(\frac{\exp \left(q \Delta_{t}(R)\right)}{|\Gamma(t, R)|} \int_{\Gamma(t, R)} \psi^{-q}(\tau)|d \tau|\right)^{1 / q}<\infty
$$

the supremum over all $t \in \Gamma$ and all $R>0$, and thus to

$$
\sup _{t \in \Gamma} \sup _{R>0}\left(e^{-p \Delta_{t}(R)} \Delta_{t}\left(\psi^{p}, 0, R\right)\right)^{1 / p}\left(e^{q \Delta_{t}(R)} \Delta_{t}\left(\psi^{-q}, 0, R\right)\right)^{1 / q}<\infty
$$

By Jensen's inequality, $e^{s \Delta_{t}(R)} \leq \Delta_{t}\left(\psi^{s}, 0, R\right)$ whenever $s \in \mathbf{R}$ and $\psi^{s} \in L^{1}(\Gamma)$. Hence,

$$
e^{-p \Delta_{t}(R)} \Delta_{t}\left(\psi^{p}, 0, R\right) \geq 1, e^{q \Delta_{t}(R)} \Delta_{t}\left(\psi^{-q}, 0, R\right) \geq 1
$$

and we deduce that (5.4) is satisfied if and only if

$$
1 \leq \sup _{t \in \Gamma} \sup _{R>0} e^{-\Delta_{t}(R)}\left(\Delta_{t}\left(\psi^{p}, 0, R\right)\right)^{1 / p}=: C_{1}<\infty
$$

and

$$
1 \leq \sup _{t \in \Gamma} \sup _{R>0} e^{\Delta_{t}(R)}\left(\Delta_{t}\left(\psi^{-q}, 0, R\right)\right)^{1 / q}=: C_{2}<\infty .
$$

Put $\Gamma^{+}(t, R):=\left\{\tau \in \Gamma(t, R): \log \psi(\tau) \geq \Delta_{t}(R)\right\}, \Gamma^{-}(t, R):=\{\tau \in \Gamma(t, R):$ $\left.\log \psi(\tau)<\Delta_{t}(R)\right\}$. Using Jensen's and Hölder's inequalities, we obtain from (5.5) 
and (5.6) that

$$
\begin{aligned}
& \exp \left(\frac{1}{|\Gamma(t, R)|} \int_{\Gamma(t, R)}\left|\log \psi(\tau)-\Delta_{t}(R)\right||d \tau|\right) \\
& \leq \frac{1}{|\Gamma(t, R)|} \int_{\Gamma(t, R)} e^{\left|\log \psi(\tau)-\Delta_{t}(R)\right|}|d \tau| \\
&=\frac{1}{|\Gamma(t, R)|}\left(\int_{\Gamma^{+}(t, R)} e^{\log \psi(\tau)-\Delta_{t}(R)}|d \tau|+\int_{\Gamma^{-}(t, R)} e^{-\left(\log \psi(\tau)-\Delta_{t}(R)\right)}|d \tau|\right) \\
& \leq\left(\frac{1}{|\Gamma(t, R)|} \int_{\Gamma(t, R)} e^{p\left(\log \psi(\tau)-\Delta_{t}(R)\right)}|d \tau|\right)^{1 / p} \\
&+\left(\frac{1}{|\Gamma(t, R)|} \int_{\Gamma(t, R)} e^{-q\left(\log \psi(\tau)-\Delta_{t}(R)\right)}|d \tau|\right)^{1 / q} \\
&= e^{-\Delta_{t}(R)}\left(\Delta_{t}\left(\psi^{p}, 0, R\right)\right)^{1 / p} e^{\Delta_{t}(R)}\left(\Delta_{t}\left(\psi^{-q}, 0, R\right)\right)^{1 / q} \leq C_{1} C_{2},
\end{aligned}
$$

which means that $\log \psi \in B M O(\Gamma)$.

The following lemma provides uniform lower and upper estimates for the four functions $V_{t}^{0} \psi, V_{t} \psi, U_{t}^{0} \psi, U_{t} \psi$ in a neighborhood of the point 1 . Notice that, obviously,

$$
\left(V_{t}^{0} \psi\right)(x) \leq\left(V_{t} \psi\right)(x), \quad\left(U_{t}^{0} \psi\right)(x) \leq\left(U_{t} \psi\right)(x)
$$

for all $x \in(0, \infty)$.

Lemma 5.4. Let $\psi: \Gamma \rightarrow[0, \infty]$ be a weight and let $x_{0} \in(1, \infty)$ be an arbitrary point.

(a) If $\log \psi \in B M O(\Gamma)$, then

$$
0<1 / C\left(x_{0}\right) \leq \inf _{t \in \Gamma} \inf _{x \in\left[1 / x_{0}, x_{0}\right]}\left(V_{t}^{0} \psi\right)(x) \leq \sup _{t \in \Gamma} \sup _{x \in\left[1 / x_{0}, x_{0}\right]}\left(V_{t} \psi\right)(x) \leq C\left(x_{0}\right)<\infty
$$

where $C\left(x_{0}\right):=\exp \left(C_{\Gamma} x_{0}\|\log \psi\|_{*}\right)$ and $C_{\Gamma}$ is the Carleson constant from (1.3).

(b) If $\psi \in A_{p}(\Gamma)(1<p<\infty)$, then

$$
0<C_{3}\left(x_{0}\right) \leq \inf _{t \in \Gamma} \inf _{x \in\left[1 / x_{0}, x_{0}\right]}\left(U_{t}^{0} \psi\right)(x) \leq \sup _{t \in \Gamma} \sup _{x \in\left[1 / x_{0}, x_{0}\right]}\left(U_{t} \psi\right)(x) \leq C_{4}\left(x_{0}\right)<\infty
$$

where

$$
C_{3}\left(x_{0}\right):=\left(1 / C\left(x_{0}\right)\right) \exp \left(-\frac{2 C_{\Gamma}}{1-\kappa}\|\log \psi\|_{*}\right), C_{4}\left(x_{0}\right):=\frac{C_{\Gamma}}{1-\kappa} C_{1} C_{2} C\left(x_{0}\right),
$$

and $C_{1}, C_{2}$ are determined by (5.5), (5.6).

(c) If $\log \psi \in B M O(\Gamma)$, then $V_{t}^{0} \psi$ and $V_{t} \psi$ are regular functions mapping $(0, \infty)$ to $(0, \infty)$, if $\psi \in A_{p}(\Gamma)(1<p<\infty)$, then $U_{t}^{0} \psi$ and $U_{t} \psi$ are regular functions mapping $(0, \infty)$ to $(0, \infty)$, and if $\psi$ is continuous and nonvanishing on $\Gamma \backslash\{t\}$ and $W_{t} \psi$ is regular, then $W_{t}^{0} \psi$ is also regular and both $W_{t}^{0} \psi$ and $W_{t} \psi$ map $(0, \infty)$ to $(0, \infty)$. 
Proof. Again let $\Delta_{t}(R):=\Delta_{t}(\log \psi, 0, R)$, where $0<R \leq d_{t}$.

(a) By the Carleson condition (1.3), we have for $x \in\left[1 / x_{0}, 1\right]$

$$
\begin{aligned}
& \left|\Delta_{t}(x R)-\Delta_{t}(R)\right|=\frac{1}{|\Gamma(t, x R)|}\left|\int_{\Gamma(t, x R)}\left(\log \psi(\tau)-\Delta_{t}(R)\right)\right| d \tau|| \\
& \leq \frac{|\Gamma(t, R)|}{|\Gamma(t, x R)|} \frac{1}{|\Gamma(t, R)|} \int_{\Gamma(t, R)}\left|\log \psi(\tau)-\Delta_{t}(R)\right||d \tau| \\
& \leq \frac{C_{\Gamma}}{x}\|\log \psi\|_{*} \leq C_{\Gamma} x_{0}\|\log \psi\|_{*}
\end{aligned}
$$

and thus

$$
\left(V_{t}^{0} \psi\right)(x)=\limsup _{R \rightarrow 0} e^{\Delta_{t}(x R)-\Delta_{t}(R)} \geq e^{-C_{\Gamma} x_{0}\|\log \psi\|_{*}}=: 1 / C\left(x_{0}\right) .
$$

Analogously, if $x \in\left[1, x_{0}\right]$, then

$$
\left|\Delta_{t}(R)-\Delta_{t}\left(x^{-1} R\right)\right| \leq C_{\Gamma} x\|\log \psi\|_{*} \leq C_{\Gamma} x_{0}\|\log \psi\|_{*},
$$

whence

$$
\left(V_{t}^{0} \psi\right)(x)=\limsup _{R \rightarrow 0} e^{\Delta_{t}(R)-\Delta_{t}\left(x^{-1} R\right)} \geq e^{-C_{\Gamma} x_{0}\|\log \psi\|_{*}}=1 / C\left(x_{0}\right) .
$$

From (5.8) and (5.9) we also get that

$$
\left(V_{t} \psi\right)(x) \leq e^{C_{\Gamma} x_{0}\|\log \psi\|_{*}}=C\left(x_{0}\right)
$$

for all $t \in \Gamma$ and all $x \in\left[1 / x_{0}, x_{0}\right]$.

(b) From Lemma 5.2 we infer that $\Delta_{t}(R)$ is well-defined for $\psi \in A_{p}(\Gamma)$. We have

$$
\begin{aligned}
& \left|\Delta_{t}(R)-\Delta_{t}(\log \psi, \kappa R, R)\right|=\left|\Delta_{t}\left(\log \psi-\Delta_{t}(R), \kappa R, R\right)\right| \\
& \leq \Delta_{t}\left(\left|\log \psi-\Delta_{t}(R)\right|, \kappa R, R\right) \\
& \leq \frac{|\Gamma(t, R)|}{|\Gamma(t, \kappa R, R)|} \frac{1}{|\Gamma(t, R)|} \int_{\Gamma(t, R)}\left|\log \psi(\tau)-\Delta_{t}(R)\right||d \tau|
\end{aligned}
$$

and since $\log \psi \in B M O(\Gamma)$ by Lemma $5.3,|\Gamma(t, R)| \leq C_{\Gamma} R$ by the Carleson condition (1.3), and $|\Gamma(t, \kappa R, R)| \geq(1-\kappa) R$, we get

$$
\left|\Delta_{t}(R)-\Delta_{t}(\log \psi, \kappa R, R)\right| \leq \frac{C_{\Gamma}}{1-\kappa}\|\log \psi\|_{*} .
$$

Jensen's inequality together with (5.10) implies that

$$
\begin{aligned}
e^{\Delta_{t}(R)} & \leq e^{\Delta_{t}(\log \psi, \kappa R, R)} e^{\left|\Delta_{t}(R)-\Delta_{t}(\log \psi, \kappa R, R)\right|} \\
& \leq\left(\Delta_{t}\left(\psi^{p}, \kappa R, R\right)\right)^{1 / p} \exp \left(\frac{C_{\Gamma}}{1-\kappa}\|\log \psi\|_{*}\right) .
\end{aligned}
$$

On the other hand, from (5.5) we infer that

$$
\left(\Delta_{t}\left(\psi^{p}, \kappa R, R\right)\right)^{1 / p} \leq\left(\frac{|\Gamma(t, R)|}{|\Gamma(t, \kappa R, R)|}\right)^{1 / p}\left(\Delta_{t}\left(\psi^{p}, 0, R\right)\right)^{1 / p} \leq\left(\frac{C_{\Gamma}}{1-\kappa}\right)^{1 / p} C_{1} e^{\Delta_{t}(R)} .
$$

Combining (5.11) and (5.12) we arrive at the conclusion that

$$
C_{3} e^{\Delta_{t}(R)} \leq\left(\Delta_{t}\left(\psi^{p}, \kappa R, R\right)\right)^{1 / p} \leq C_{4} e^{\Delta_{t}(R)}
$$


with

$$
C_{3}:=\exp \left(-\frac{C_{\Gamma}}{1-\kappa}\|\log \psi\|_{*}\right), C_{4}:=\left(\frac{C_{\Gamma}}{1-\kappa}\right)^{1 / p} C_{1} .
$$

Analogously one can show that

$$
C_{3} e^{-\Delta_{t}(R)} \leq\left(\Delta_{t}\left(\psi^{-q}, \kappa R, R\right)\right)^{1 / q} \leq C_{5} e^{-\Delta_{t}(R)}
$$

with $C_{5}:=\left(C_{\Gamma} /(1-\kappa)\right)^{1 / q} C_{2}$. Consequently, if $x \in(0,1]$, then

$$
\begin{aligned}
\left(U_{t}^{0} \psi\right)(x) & =\limsup _{x \rightarrow 0}\left(\Delta_{t}\left(\psi^{p}, \kappa x R, x R\right)\right)^{1 / p}\left(\Delta_{t}\left(\psi^{-q}, \kappa R, R\right)\right)^{1 / q} \\
& \geq \limsup _{x \rightarrow 0}\left(C_{3}^{2} e^{\Delta_{t}(x R)-\Delta_{t}(R)}\right)
\end{aligned}
$$

and from (5.8) we deduce that

$$
\left(U_{t}^{0} \psi\right)(x) \geq C_{3}^{2} e^{-C_{\Gamma} x_{0}\|\log \psi\|_{*}}=: C_{3}\left(x_{0}\right) .
$$

Using (5.9) one analogously gets $(5.15)$ for $x \in\left[1, x_{0}\right]$. Finally, in the same way one can show that

$$
\left(U_{t} \psi\right)(x) \leq C_{4} C_{5} e^{C_{\Gamma} x_{0}\|\log \psi\|_{*}}=: C_{4}\left(x_{0}\right) \text { for } x \in\left[1 / x_{0}, x_{0}\right] .
$$

(c) In view of (5.7), the assertions for $V_{t}^{0} \psi, V_{t} \psi$ and $U_{t}^{0} \psi, U_{t} \psi$ are immediate from (a) and (b), respectively. The claim made for $W_{t} \psi$ and $W_{t}^{0} \psi$ follows from Lemmas 6.3 and 6.4 of [5].

Lemma 5.5. Under the conditions of Lemma 5.4(c), the six functions $V_{t} \psi, V_{t}^{0} \psi$, $U_{t} \psi, U_{t}^{0} \psi, W_{t} \psi, W_{t}^{0} \psi$ are regular submultiplicative functions mapping $(0, \infty)$ to $(0, \infty)$.

Proof. Let us consider $U_{t} \psi$. Lemma 5.4(c) tells us that $U_{t} \psi(x) \in(0, \infty)$ for all $x \in(0, \infty)$ and that $U_{t} \psi$ is regular. Suppose $x_{1}, x_{2} \in(0, \infty)$ and $x_{1} x_{2} \in(0,1]$. We then have

$$
\begin{aligned}
& \left(U_{t} \psi\right)\left(x_{1} x_{2}\right)=\sup _{R>0}\left(\Delta_{t}\left(\psi^{p}, \kappa x_{1} x_{2} R, x_{1} x_{2} R\right)\right)^{1 / p}\left(\Delta_{t}\left(\psi^{-q}, \kappa R, R\right)\right)^{1 / q} \\
& \leq \sup _{R>0}\left[\left(\Delta_{t}\left(\psi^{p}, \kappa x_{1} x_{2} R, x_{1} x_{2} R\right)\right)^{1 / p}\left(\Delta_{t}\left(\psi^{-q}, \kappa x_{2} R, x_{2} R\right)\right)^{1 / q}\right] \\
& \times \sup _{R>0}\left[\left(\Delta_{t}\left(\psi^{p}, \kappa x_{2} R, x_{2} R\right)\right)^{1 / p}\left(\Delta_{t}\left(\psi^{-q}, \kappa R, R\right)\right)^{1 / q}\right] .
\end{aligned}
$$

If $x_{1}, x_{2} \in(0,1]$, then the first factor on the right of (5.16) equals

$$
\begin{aligned}
& \sup _{0<R \leq x_{2} d_{t}}\left[\left(\Delta_{t}\left(\psi^{p}, \kappa x_{1} R, x_{1} R\right)\right)^{1 / p}\left(\Delta_{t}\left(\psi^{-q}, \kappa R, R\right)\right)^{1 / q}\right] \\
\leq & \sup _{0<R \leq d_{t}}\left[\left(\Delta_{t}\left(\psi^{p}, \kappa x_{1} R, x_{1} R\right)\right)^{1 / p}\left(\Delta_{t}\left(\psi^{-q}, \kappa R, R\right)\right)^{1 / q}\right]=\left(U_{t} \psi\right)\left(x_{1}\right)
\end{aligned}
$$

and hence, the right-hand side of (5.16) is not greater than $\left(U_{t} \psi\right)\left(x_{1}\right)\left(U_{t} \psi\right)\left(x_{2}\right)$. Suppose $x_{1} \in(1, \infty)$ and $x_{2} \in(0,1]$. Then the second factor on the right of $(5.16)$ is $\left(U_{t} \psi\right)\left(x_{2}\right)$ and the first factor equals

$$
\begin{aligned}
& \sup _{0<R \leq x_{1} x_{2} d_{t}}\left[\left(\Delta_{t}\left(\psi^{p}, \kappa R, R\right)\right)^{1 / p}\left(\Delta_{t}\left(\psi^{-q}, \kappa x_{1}^{-1} R, x_{1}^{-1} R\right)\right)^{1 / q}\right] \\
& \leq \sup _{0<R \leq d_{t}}\left[\left(\Delta_{t}\left(\psi^{p}, \kappa R, R\right)\right)^{1 / p}\left(\Delta_{t}\left(\psi^{-q}, \kappa x_{1}^{-1} R, x_{1}^{-1} R\right)\right)^{1 / q}\right]=\left(U_{t} \psi\right)\left(x_{1}\right) .
\end{aligned}
$$


This settles the case $x_{1} x_{2} \in(0,1]$. For $x_{1} x_{2} \in(1, \infty)$ the proof is analogous. Thus, $U_{t} \psi$ is submultiplicative.

The submultiplicativity of $V_{t} \psi$ can be shown similarly. Taking into account that $U_{t}^{0} \psi$ and $V_{t}^{0} \psi$ map $(0, \infty)$ to $(0, \infty)$ by virtue of Lemma $5.4(\mathrm{c})$, we obtain in the same way that $U_{t}^{0} \psi$ and $V_{t}^{0} \psi$ are regular submultiplicative functions. The assertions concerning $W_{t} \psi$ and $W_{t}^{0} \psi$ are Lemmas 6.3 and 6.4 of [5].

Given a weight $\psi$ such that $\psi \in L^{p}(\Gamma)$ and $\psi^{-1} \in L^{q}(\Gamma)$, we may define $U \psi, U^{0} \psi:(0, \infty) \rightarrow[0, \infty]$ by

$$
(U \psi)(x):=\sup _{t \in \Gamma}\left(U_{t} \psi\right)(x), \quad\left(U^{0} \psi\right)(x):=\sup _{t \in \Gamma}\left(U_{t}^{0} \psi\right)(x) .
$$

If $\psi$ is a weight for which $\log \psi \in L^{1}(\Gamma)$, we denote by $V \psi, V^{0} \psi:(0, \infty) \rightarrow[0, \infty]$ the functions given by

$$
(V \psi)(x):=\sup _{t \in \Gamma}\left(V_{t} \psi\right)(x), \quad\left(V^{0} \psi\right)(x):=\sup _{t \in \Gamma}\left(V_{t}^{0} \psi\right)(x) .
$$

Theorem 5.6. Let $\psi: \Gamma \rightarrow[0, \infty]$ be a weight.

(a) If $\log \psi \in B M O(\Gamma)$, then $V_{t} \psi, V_{t}^{0} \psi(t \in \Gamma)$ and $V \psi, V^{0} \psi$ are regular submultiplicative functions mapping $(0, \infty)$ to $(0, \infty)$.

(b) If even $\psi \in A_{p}(\Gamma)(1<p<\infty)$, then $U_{t} \psi, U_{t}^{0} \psi, V_{t} \psi, V_{t}^{0} \psi(t \in \Gamma)$ as well as $U \psi, U^{0} \psi, V \psi, V^{0} \psi$ are regular submultiplicative functions of $(0, \infty)$ to $(0, \infty)$.

Proof. Lemmas 5.3, 5.4, 5.5.

The question about the regularity of $W_{t} \psi$ and thus $W_{t}^{0} \psi$ for weights $\psi$ belonging to $C(\Gamma \backslash\{t\})$ is more difficult. For our purposes the following simple result, which concerns weights "parametrized by the radius $r=|\tau-t|$ ", will suffice.

Proposition 5.7. Let $\Gamma$ be a Carleson Jordan curve and $t \in \Gamma$. Assume $\psi(\tau)=$ $e^{F(|\tau-t|)}(\tau \in \Gamma \backslash\{t\})$ where $F:\left(0, d_{t}\right] \rightarrow \mathbf{R}$ is continuous on $\left(0, d_{t}\right]$ and continuously differentiable on $\left(0, d_{t}\right)$, denote by a dot the derivative $d / d r$, and suppose $r \dot{F}(r)$ is bounded for $r \in\left(0, d_{t}\right)$. Then $W_{t} \psi$ and $W_{t}^{0} \psi$ are regular submultiplicative functions of $(0, \infty)$ to $(0, \infty)$.

Proof. For $x \in(0,1)$ we have

$$
\max _{|\tau-t|=x R} \psi(\tau) / \min _{|\tau-t|=R} \psi(\tau)=e^{F(x R)-F(R)}=e^{\dot{F}(\xi R)(x-1) R}
$$

with some $\xi \in(x, 1)$. Clearly, $\dot{F}(\xi R)(x-1) R=\xi R \dot{F}(\xi R)(x-1) / \xi$ is bounded for $x$ in $[1 / 2,1)$. An analogous reasoning for $x \in(1,2]$ and the equality

$$
\max _{|\tau-t|=R} \psi(\tau) / \min _{|\tau-t|=R} \psi(\tau)=e^{F(R)-F(R)}=1
$$

give the regularity of $W_{t} \psi$ and $W_{t}^{0} \psi$. Their submultiplicativity and the fact that they map into $(0, \infty)$ then follow from Lemma 5.5.

In particular, if $d_{t}=1$ and $F(r)=\lambda \log r$ with $\lambda \in \mathbf{R}$, in which case $\psi(\tau)=$ $|\tau-t|^{\lambda}$, then $W_{t} \psi$ and $W_{t}^{0} \psi$ are regular and submultiplicative. Notice that $|\tau-t|^{\lambda}$ is a weight in $A_{p}(\Gamma)$ if and only if $-1 / p<\lambda<1 / q$. When proving Theorem 3.4 we will employ functions $F$ of the form

$$
F(r)=f(\log (-\log r)) \log r, \quad r \in(0,1) .
$$


In that case

$$
r \dot{F}(r)=f^{\prime}(\log (-\log r))+f(\log (-\log r))
$$

and hence $r \dot{F}(r)$ is bounded whenever $f \in C^{1}(\mathbf{R})$ and $f$ as well as $f^{\prime}$ is bounded on $\mathbf{R}$.

Under the hypotheses of Theorem 5.6 or Proposition 5.7 we may define the lower and upper indices of the corresponding regular submultiplicative functions by the formulas of Theorem 5.1(a):

$$
\alpha\left(U_{t} \psi\right):=\lim _{x \rightarrow 0} \frac{\log \left(U_{t} \psi\right)(x)}{\log x}, \beta\left(U_{t} \psi\right):=\lim _{x \rightarrow \infty} \frac{\log \left(U_{t} \psi\right)(x)}{\log x}, \ldots
$$

Lemma 5.8. If $\psi \in A_{p}(\Gamma)(1<p<\infty)$, then

$$
\begin{aligned}
& \alpha\left(U_{t} \psi\right)=\alpha\left(V_{t} \psi\right) \leq \beta\left(V_{t} \psi\right)=\beta\left(U_{t} \psi\right) \text { for all } t \in \Gamma \\
& \alpha(U \psi)=\alpha(V \psi) \leq \beta(V \psi)=\beta(U \psi)
\end{aligned}
$$

Proof. We have

$$
\alpha\left(V_{t} \psi\right)=\lim _{x \rightarrow 0} \frac{1}{\log x} \sup _{R>0}\left(\Delta_{t}(x R)-\Delta_{t}(R)\right)
$$

where $\Delta_{t}(r):=\Delta_{t}(\log \psi, 0, r)$, and

$$
\alpha\left(U_{t} \psi\right)=\lim _{x \rightarrow 0} \frac{1}{\log x} \sup _{R>0}\left(\frac{1}{p} \log \Delta_{t}\left(\psi^{p}, \kappa x R, x R\right)+\frac{1}{q} \log \Delta_{t}\left(\psi^{-q}, \kappa R, R\right)\right) .
$$

From (5.13) and (5.14) we immediately get the equality $\alpha\left(V_{t} \psi\right)=\alpha\left(U_{t} \psi\right)$. The equality $\beta\left(V_{t} \psi\right)=\beta\left(U_{t} \psi\right)$ follows analogously. Since $\alpha(\varrho) \leq \beta(\varrho)$ for every regular submultiplicative function, we arrive at (5.20). In the same way one can show (5.21).

The previous lemma shows in particular that the indices of $U_{t} \psi$ and $U \psi$ for $\psi \in A_{p}(\Gamma)$ do not depend on the parameter $\kappa \in(0,1)$.

Lemma 5.9. If $\psi \in A_{p}(\Gamma)(1<p<\infty)$, then

$$
\alpha\left(V_{t} \psi\right)=\alpha\left(V_{t}^{0} \psi\right) \leq \beta\left(V_{t}^{0} \psi\right)=\beta\left(V_{t} \psi\right)
$$

Proof. This can be proved by the argument used in the proof of Lemma 6.4 of [5] (which in turn mimics the proofs of Lemma 2(a) of [7] and Theorem 8.18 of [3]).

Lemma 5.10. Let $\psi$ be as in Proposition 5.7 with $F$ given by (5.19). If $f \in C^{2}(\mathbf{R})$ and the functions $f, f^{\prime}, f^{\prime \prime}$ are bounded on $\mathbf{R}$, then $W_{t} \psi$ and $W_{t}^{0} \psi$ are regular and

$$
\begin{aligned}
& \alpha\left(W_{t} \psi\right)=\alpha\left(W_{t}^{0} \psi\right)=\liminf _{r \rightarrow 0} r \dot{F}(r)=\liminf _{y \rightarrow \infty}\left(f(y)+f^{\prime}(y)\right), \\
& \beta\left(W_{t} \psi\right)=\beta\left(W_{t}^{0} \psi\right)=\limsup _{r \rightarrow 0} r \dot{F}(r)=\limsup _{y \rightarrow \infty}\left(f(y)+f^{\prime}(y)\right) .
\end{aligned}
$$

Proof. This follows from Proposition 5.7 and from Lemmas 4.2 and 6.4 of [5]. 
6. MudKenhoupt CONDition AND INDiCES OF SUBMUltiplicative FUnCTIONS

Theorem 6.1. Let $\Gamma$ be a Carleson Jordan curve, let $p \in(1, \infty)$, and let $\psi: \Gamma \rightarrow$ $[0, \infty]$ be a weight. Suppose $\psi \in L_{\mathrm{loc}}^{p}(\Gamma \backslash\{t\})$ and $\psi^{-1} \in L_{\mathrm{loc}}^{q}(\Gamma \backslash\{t\})$. If the submultiplicative function $U_{t} \psi$ is regular and

$$
-1 / p<\alpha\left(U_{t} \psi\right) \leq \beta\left(U_{t} \psi\right)<1 / q,
$$

then

$$
B_{t}(\psi):=\sup _{R>0}\left(\frac{1}{R} \int_{\Gamma(t, R)} \psi^{p}(\tau)|d \tau|\right)^{1 / p}\left(\frac{1}{R} \int_{\Gamma(t, R)} \psi^{-q}(\tau)|d \tau|\right)^{1 / q}<\infty .
$$

Proof. Put $\alpha=\alpha\left(U_{t} \psi\right)$ and $\beta=\beta\left(U_{t} \psi\right)$. By (6.1), there is an $\varepsilon>0$ such that $-1 / p<\alpha-\varepsilon<\beta+\varepsilon<1 / q$ and hence

$$
1+p(\alpha-\varepsilon)>0, \quad 1-q(\beta+\varepsilon)>0 .
$$

Recall that $U_{t} \psi$ involves a parameter $\kappa \in(0,1)$. From Theorem 5.1(c) we deduce that, for some $n_{0} \geq 0$,

$$
\begin{aligned}
& \left(U_{t} \psi\right)\left(\kappa^{n}\right) \leq \kappa^{n(\alpha-\varepsilon)} \text { for all } n \geq n_{0}, \\
& \left(U_{t} \psi\right)\left(\kappa^{n}\right) \leq \kappa^{n(\beta+\varepsilon)} \text { for all } n \leq-n_{0},
\end{aligned}
$$

and from the regularity and submultiplicativity of $U_{t} \psi$ we get

$$
\left(U_{t} \psi\right)\left(\kappa^{n}\right) \leq M:=\max _{-n_{0}<n<n_{0}}\left(U_{t} \psi\right)\left(\kappa^{n}\right)<\infty \text { for }-n_{0}<n<n_{0} .
$$

The Carleson condition (1.3) implies that $r \leq|\Gamma(t, r)| \leq C_{\Gamma} r$ for all $r \in\left(0, d_{t}\right]$, whence

$$
\left|\Gamma\left(t, \kappa^{n+1} R, \kappa^{n} R\right)\right| \leq\left|\Gamma\left(t, \kappa^{n} R\right)\right|-\left|\Gamma\left(t, \kappa^{n+1} R\right)\right| \leq C_{\Gamma} \kappa^{n} R-\kappa^{n+1} R=c_{0} \kappa^{n} R
$$

with $c_{0}:=C_{\Gamma}-\kappa>0$. The definition (5.1) gives

$$
\begin{aligned}
& \Delta_{t}\left(\psi^{p}, \kappa^{n+1} R, \kappa^{n} R\right) \leq\left(U_{t} \psi\right)^{p}\left(\kappa^{n}\right)\left(\Delta_{t}\left(\psi^{-q}, \kappa R, R\right)\right)^{-p / q} \text { for all } n \geq 0, \\
& \Delta_{t}\left(\psi^{-q}, \kappa^{n+1} R, \kappa^{n} R\right) \leq\left(U_{t} \psi\right)^{q}\left(\kappa^{-n}\right)\left(\Delta_{t}\left(\psi^{p}, \kappa R, R\right)\right)^{-q / p} \text { for all } n \geq 0 .
\end{aligned}
$$

Since

$$
0<\kappa^{1+p(\alpha-\varepsilon)}<1, \quad 0<\kappa^{1-q(\beta+\varepsilon)}<1
$$

by virtue of (6.3), we obtain from (6.4)-(6.9) that

$$
\begin{aligned}
& \int_{\Gamma(t, R)} \psi^{p}(\tau)|d \tau|=\sum_{n=0}^{\infty}\left|\Gamma\left(t, \kappa^{n+1} R, \kappa^{n} R\right)\right| \Delta_{t}\left(\psi^{p}, \kappa^{n+1} R, \kappa^{n} R\right) \\
\leq & \sum_{n=0}^{\infty} c_{0} \kappa^{n} R\left(U_{t} \psi\right)^{p}\left(\kappa^{n}\right)\left(\Delta_{t}\left(\psi^{-q}, \kappa R, R\right)\right)^{-p / q} \\
\leq & c_{0} R\left(\sum_{n=n_{0}}^{\infty} \kappa^{n(1+p(\alpha-\varepsilon))}+\sum_{n=0}^{n_{0}-1} \kappa^{n} M^{p}\right)\left(\Delta_{t}\left(\psi^{-q}, \kappa R, R\right)\right)^{-p / q} \\
= & : R C_{6}\left(\Delta_{t}\left(\psi^{-q}, \kappa R, R\right)\right)^{-p / q} \text { with } C_{6}<\infty
\end{aligned}
$$


and analogously,

$$
\int_{\Gamma(t, R)} \psi^{-q}(\tau)|d \tau| \leq R C_{7}\left(\Delta_{t}\left(\psi^{p}, \kappa R, R\right)\right)^{-q / p}
$$

with

$$
C_{7}:=c_{0}\left(\sum_{n=n_{0}}^{\infty} \kappa^{n(1-q(\beta+\varepsilon))}+\sum_{n=0}^{n_{0}-1} \kappa^{n} M^{q}\right)<\infty
$$

Multiplying (6.10) and (6.11) and applying Hölder's inequality, we get

$$
\begin{aligned}
& \left(\frac{1}{R} \int_{\Gamma(t, R)} \psi^{p}(\tau)|d \tau|\right)^{1 / p}\left(\frac{1}{R} \int_{\Gamma(t, R)} \psi^{-q}(\tau)|d \tau|\right)^{1 / q} \\
& \leq C_{6}^{1 / p} C_{7}^{1 / q}\left(\Delta_{t}\left(\psi^{-q}, \kappa R, R\right)\right)^{-1 / q}\left(\Delta_{t}\left(\psi^{p}, \kappa R, R\right)\right)^{-1 / p} \leq C_{6}^{1 / p} C_{7}^{1 / q},
\end{aligned}
$$

which gives (6.2).

Recall the definition (5.17) of $U \psi$. The following two results provide useful tools for checking whether a weight $\psi$ satisfies the Muckenhoupt condition.

Theorem 6.2. Let $\Gamma$ be a Carleson Jordan curve and let $\psi: \Gamma \rightarrow[0, \infty]$ be a weight such that $\psi \in L^{p}(\Gamma)$ and $\psi^{-1} \in L^{q}(\Gamma)$. Then $\psi \in A_{p}(\Gamma)(1<p<\infty)$ if and only if

(i) the submultiplicative function $U \psi$ given by (5.17) is regular,

(ii) $-1 / p<\alpha(U \psi) \leq \beta(U \psi)<1 / q$.

Proof. Suppose (i) and (ii) hold. Repeating the proof of Theorem 6.1 with $U_{t} \psi$ replaced by $U \psi$ we arrive at the estimate $B_{t}(\psi) \leq C_{6}^{1 / p} C_{7}^{1 / q}$ with certain constants $C_{6}$ and $C_{7}$ independent of $t \in \Gamma$, which implies that $\psi \in A_{p}(\Gamma)$.

Conversely, suppose $\psi \in A_{p}(\Gamma)$. Then condition (i) follows from Theorem 5.6(b). Put $\alpha=\alpha(U \psi)$ and $\beta=\beta(U \psi)$. Assume that $\alpha<-1 / p$. By Theorem 5.1(b),

$$
(U \psi)\left(\kappa^{n}\right) \geq \kappa^{n \alpha} \text { for } n \geq 0, \quad(U \psi)\left(\kappa^{n}\right) \geq \kappa^{n \beta} \text { for } n \leq 0,
$$

where $\kappa \in(0,1)$ is the parameter entering the definition (5.1). Fix $\varepsilon \in(0,1)$. From (5.1) and (5.17) we infer that for every $n \geq 0$ there exist $t_{n} \in \Gamma$ and $R_{n} \in\left(0, d_{t}\right]$ such that

$$
\left(\Delta_{t_{n}}\left(\psi^{p}, \kappa^{n+1} R_{n}, \kappa^{n} R_{n}\right)\right)^{1 / p} \geq(1-\varepsilon)(U \psi)\left(\kappa^{n}\right)\left(\Delta_{t_{n}}\left(\psi^{-q}, \kappa R_{n}, R_{n}\right)\right)^{-1 / q} .
$$

This and (6.12) give

$$
\left(\Delta_{t_{n}}\left(\psi^{p}, \kappa^{n+1} R_{n}, \kappa^{n} R_{n}\right)\right)^{1 / p} \geq(1-\varepsilon) \kappa^{n \alpha}\left(\Delta_{t_{n}}\left(\psi^{-q}, \kappa R_{n}, R_{n}\right)\right)^{-1 / q},
$$


whence

$$
\begin{aligned}
& \left(\frac{1}{R_{n}} \int_{\Gamma\left(t_{n}, R_{n}\right)} \psi^{p}(\tau)|d \tau|\right)^{1 / p} \\
& \geq\left(\frac{\left|\Gamma\left(t_{n}, \kappa^{n+1} R_{n}, \kappa^{n} R_{n}\right)\right|}{R_{n}} \Delta_{t_{n}}\left(\psi^{p}, \kappa^{n+1} R_{n}, \kappa^{n} R_{n}\right)\right)^{1 / p} \\
& \geq\left(\frac{(1-\kappa) \kappa^{n} R_{n}}{R_{n}}\right)^{1 / p}\left(\Delta_{t_{n}}\left(\psi^{p}, \kappa^{n+1} R_{n}, \kappa^{n} R_{n}\right)\right)^{1 / p} \\
& \geq(1-\kappa)^{1 / p}(1-\varepsilon) \kappa^{n(1 / p+\alpha)}\left(\Delta_{t_{n}}\left(\psi^{-q}, \kappa R_{n}, R_{n}\right)\right)^{-1 / q}
\end{aligned}
$$

On the other hand,

$$
\begin{aligned}
& \left(\frac{1}{R_{n}} \int_{\Gamma\left(t_{n}, R_{n}\right)} \psi^{-q}(\tau)|d \tau|\right)^{1 / q} \geq\left(\frac{\left|\Gamma\left(t_{n}, \kappa R_{n}, R_{n}\right)\right|}{R_{n}} \Delta_{t_{n}}\left(\psi^{-q}, \kappa R_{n}, R_{n}\right)\right)^{1 / q} \\
& \geq(1-\kappa)^{1 / q}\left(\Delta_{t_{n}}\left(\psi^{-q}, \kappa R_{n}, R_{n}\right)\right)^{1 / q}
\end{aligned}
$$

From (6.13) and (6.14) we obtain that

$$
\begin{aligned}
B & :=\sup _{t \in \Gamma} \sup _{R>0}\left(\frac{1}{R} \int_{\Gamma(t, R)} \psi^{p}(\tau)|d \tau|\right)^{1 / p}\left(\frac{1}{R} \int_{\Gamma(t, R)} \psi^{-q}(\tau)|d \tau|\right)^{1 / q} \\
& \geq\left(\frac{1}{R_{n}} \int_{\Gamma\left(t_{n}, R_{n}\right)} \psi^{p}(\tau)|d \tau|\right)^{1 / p}\left(\frac{1}{R_{n}} \int_{\Gamma\left(t_{n}, R_{n}\right)} \psi^{-q}(\tau)|d \tau|\right)^{1 / q} \\
& \geq(1-\kappa)(1-\varepsilon) \kappa^{n(1 / p+\alpha)}
\end{aligned}
$$

and since $\kappa \in(0,1)$ and $1 / p+\alpha<0$, it follows that $\kappa^{n(1 / p+\alpha)} \rightarrow \infty$ as $n \rightarrow \infty$, implying that $B=\infty$ and thus $\psi \notin A_{p}(\Gamma)$. This contradiction proves that $\alpha \geq$ $-1 / p$. Analogously one can show that $\beta \leq 1 / q$.

Finally, assume $\psi \in A_{p}(\Gamma)$ but $\alpha=-1 / p$ or $\beta=1 / q$. By a theorem of Simonenko [32], there exists an $\varepsilon>0$ such that $\psi^{1+\varepsilon} \in A_{p}(\Gamma)$. From Lemma 5.8 we deduce that

$$
\alpha\left(U \psi^{1+\varepsilon}\right)=\alpha\left(V \psi^{1+\varepsilon}\right)=(1+\varepsilon) \alpha(V \psi)=(1+\varepsilon) \alpha(U \psi)=(1+\varepsilon) \alpha
$$

and, analogously, $\beta\left(U \psi^{1+\varepsilon}\right)=(1+\varepsilon) \beta$. Since $(1+\varepsilon) \alpha<-1 / p$ or $(1+\varepsilon) \beta>1 / q$, from what has already been proved we see that $\psi^{1+\varepsilon} \notin A_{p}(\Gamma)$. This contradiction completes the proof.

Theorem 6.3. Let $\Gamma$ be a Carleson Jordan curve, let $t \in \Gamma$, and let $\psi: \Gamma \backslash\{t\} \rightarrow$ $(0, \infty)$ be a weight which is continuous and nonzero on $\Gamma \backslash\{t\}$. Also suppose $W_{t} \psi$ is regular. Then $W_{t}^{0} \psi$ is also regular and

$$
\alpha\left(W_{t} \psi\right)=\alpha\left(W_{t}^{0} \psi\right) \leq \beta\left(W_{t}^{0} \psi\right)=\beta\left(W_{t} \psi\right) .
$$

Moreover, $\psi \in A_{p}(\Gamma)(1<p<\infty)$ if and only if

$$
-1 / p<\alpha\left(W_{t} \psi\right) \leq \beta\left(W_{t} \psi\right)<1 / q .
$$

Proof. Lemma 6.4 and Theorem 7.1 of [5]. 


\section{THE INDICATOR SET}

Throughout the following sections, let $\Gamma$ be a Carleson Jordan curve, $1<p<\infty$, and $\omega \in A_{p}(\Gamma)$. For a complex number $\gamma$, define $\varphi_{t, \gamma}: \Gamma \backslash\{t\} \rightarrow(0, \infty)$ and $\eta_{t}: \Gamma \backslash\{t\} \rightarrow(0, \infty)$ by $(3.21)$ and (3.3). We so may write $\varphi_{t, \gamma}=\varphi_{t, \operatorname{Re} \gamma} \eta_{t}^{\operatorname{Im} \gamma}$. The purpose of this section is to describe the indicator set $N_{t}=N_{t}(\Gamma, p, \omega)$ given by (3.22) in terms of the transformation $U_{t}$.

Lemma 7.1. The function $W_{t} \varphi_{t, \gamma}$ is regular for every $\gamma \in \mathbf{C}$ and

$$
\begin{aligned}
\alpha\left(W_{t} \varphi_{t, \gamma}\right) & =\operatorname{Re} \gamma+\min \left\{\delta_{t}^{-} \operatorname{Im} \gamma, \delta_{t}^{+} \operatorname{Im} \gamma\right\}, \\
\beta\left(W_{t} \varphi_{t, \gamma}\right) & =\operatorname{Re} \gamma+\max \left\{\delta_{t}^{-} \operatorname{Im} \gamma, \delta_{t}^{+} \operatorname{Im} \gamma\right\}
\end{aligned}
$$

where

$$
\delta_{t}^{-}:=\alpha\left(W_{t} \eta_{t}\right), \quad \delta_{t}^{+}:=\beta\left(W_{t} \eta_{t}\right) .
$$

Proof. See the proof of Theorem 7.2 of [5].

Clearly, $\varphi_{t, \gamma} \omega \in L_{\mathrm{loc}}^{p}(\Gamma \backslash\{t\})$ and $\varphi_{t, \gamma}^{-1} \omega^{-1} \in L_{\mathrm{loc}}^{q}(\Gamma \backslash\{t\})$. Thus, by the proof of Lemma 5.5, $U_{t} \varphi_{t, \gamma} \omega$ is a well-defined submultiplicative function for every $\gamma \in \mathbf{C}$.

Lemma 7.2. The function $U_{t} \varphi_{t, \gamma} \omega$ is regular for every $\gamma \in \mathbf{C}$ and

$$
\alpha\left(U_{t} \varphi_{t, \gamma} \omega\right)=\operatorname{Re} \gamma+\alpha\left(U_{t} \eta_{t}^{\operatorname{Im} \gamma} \omega\right), \beta\left(U_{t} \varphi_{t, \gamma} \omega\right)=\operatorname{Re} \gamma+\beta\left(U_{t} \eta_{t}^{\operatorname{Im} \gamma} \omega\right) .
$$

Proof. For $x \in(0,1]$,

$$
\begin{aligned}
& \left(W_{t} \varphi_{t,-\gamma}\right)(x)=\sup _{R>0} \frac{\max \left\{\left|(\tau-t)^{-\gamma}\right|:|\tau-t|=x R\right\}}{\min \left\{\left|(\tau-t)^{-\gamma}\right|:|\tau-t|=R\right\}} \\
& =\sup _{R>0} \frac{\max \left\{\left|(\tau-t)^{\gamma}\right|:|\tau-t|=R\right\}}{\min \left\{\left|(\tau-t)^{\gamma}\right|:|\tau-t|=x R\right\}}=\left(W_{t} \varphi_{t, \gamma}\right)\left(x^{-1}\right),
\end{aligned}
$$

and similarly one gets

$$
\left(W_{t} \varphi_{t,-\gamma}\right)(x)=\left(W_{t} \varphi_{t, \gamma}\right)\left(x^{-1}\right) \text { for } x \in[1, \infty) .
$$

Thus, for every $R \in\left(0, d_{t}\right]$ and every $c \in(0,1]$ we have

$$
\begin{aligned}
\max _{|\tau-t|=c R}\left|(\tau-t)^{\gamma}\right| & \leq\left(W_{t} \varphi_{t, \gamma}\right)(c) \min _{|\tau-t|=R}\left|(\tau-t)^{\gamma}\right|, \\
\max _{|\tau-t|=c R}\left|(\tau-t)^{-\gamma}\right| & \leq\left(W_{t} \varphi_{t,-\gamma}\right)(c) \min _{|\tau-t|=R}\left|(\tau-t)^{-\gamma}\right| \\
& =\left(W_{t} \varphi_{t, \gamma}\right)\left(c^{-1}\right) \min _{|\tau-t|=R}\left|(\tau-t)^{-\gamma}\right| .
\end{aligned}
$$

Using (7.7) and (7.8) we obtain that, for every $x \in(0,1]$,

$$
\begin{aligned}
& \left(\Delta_{t}\left(\varphi_{t, \gamma}^{p} \omega^{p}, \kappa x R, x R\right)\right)^{1 / p} \\
& \leq\left(\sup _{c \in[\kappa x, x)} \max _{|\tau-t|=c R}\left|(\tau-t)^{\gamma}\right|\right)\left(\Delta_{t}\left(\omega^{p}, \kappa x R, x R\right)\right)^{1 / p} \\
& \leq\left(\sup _{c \in[\kappa x, x)}\left(W_{t} \varphi_{t, \gamma}\right)(c)\right)\left(\min _{|\tau-t|=R}\left|(\tau-t)^{\gamma}\right|\right)\left(\Delta_{t}\left(\omega^{p}, \kappa x R, x R\right)\right)^{1 / p}
\end{aligned}
$$


and

$$
\begin{aligned}
& \left(\Delta_{t}\left(\varphi_{t, \gamma}^{-q} \omega^{-q}, \kappa R, R\right)\right)^{1 / q} \\
& \leq\left(\sup _{c \in[\kappa, 1)} \max _{|\tau-t|=c R}\left|(\tau-t)^{-\gamma}\right|\right)\left(\Delta_{t}\left(\omega^{-q}, \kappa R, R\right)\right)^{1 / q} \\
& \leq\left(\sup _{c \in[\kappa, 1)}\left(W_{t} \varphi_{t, \gamma}\right)\left(c^{-1}\right)\right)\left(\min _{|\tau-t|=R}\left|(\tau-t)^{-\gamma}\right|\right)\left(\Delta_{t}\left(\omega^{-q}, \kappa R, R\right)\right)^{1 / q} .
\end{aligned}
$$

Multiplication of (7.9) and (7.10) yields

$$
\begin{aligned}
& \left(\Delta_{t}\left(\varphi_{t, \gamma}^{p} \omega^{p}, \kappa x R, x R\right)\right)^{1 / p}\left(\Delta_{t}\left(\varphi_{t, \gamma}^{-q} \omega^{-q}, \kappa R, R\right)\right)^{1 / q} \\
& \leq\left(\sup _{c \in[\kappa x, x)}\left(W_{t} \varphi_{t, \gamma}\right)(c)\right)\left(\sup _{c \in[\kappa, 1)}\left(W_{t} \varphi_{t, \gamma}\right)\left(c^{-1}\right)\right) \\
& \times\left(\Delta_{t}\left(\omega^{p}, \kappa x R, x R\right)\right)^{1 / p}\left(\Delta_{t}\left(\omega^{-q}, \kappa R, R\right)\right)^{1 / q}
\end{aligned}
$$

and taking the supremum over $R \in\left(0, d_{t}\right]$ we arrive at the inequality

$$
\left(U_{t} \varphi_{t, \gamma} \omega\right)(x) \leq\left(\sup _{c \in[\kappa x, x)}\left(W_{t} \varphi_{t, \gamma}\right)(c)\right)\left(\sup _{c \in[\kappa, 1)}\left(W_{t} \varphi_{t, \gamma}\right)\left(c^{-1}\right)\right)\left(U_{t} \omega\right)(x)
$$

for $x \in(0,1]$. Analogously one can show that

$$
\left(U_{t} \varphi_{t, \gamma} \omega\right)(x) \leq\left(\sup _{c \in[\kappa, 1)}\left(W_{t} \varphi_{t, \gamma}\right)(c)\right)\left(\sup _{c \in\left[\kappa x^{-1}, x^{-1}\right)}\left(W_{t} \varphi_{t, \gamma}\right)\left(c^{-1}\right)\right)\left(U_{t} \omega\right)(x)
$$

for $x \in[1, \infty)$. Because

$$
\begin{aligned}
& \sup _{c \in[\kappa, 1)}\left(W_{t} \varphi_{t, \gamma}\right)\left(c^{-1}\right)=\sup _{c \in\left(1, \kappa^{-1}\right]}\left(W_{t} \varphi_{t, \gamma}\right)(c) \leq \sup _{c \in\left[\kappa, \kappa^{-1}\right]}\left(W_{t} \varphi_{t, \gamma}\right)(c), \\
& \sup _{c \in\left[\kappa x^{-1}, x^{-1}\right)}\left(W_{t} \varphi_{t, \gamma}\right)\left(c^{-1}\right)=\sup _{c \in\left(x, \kappa^{-1} x\right]}\left(W_{t} \varphi_{t, \gamma}\right)(c) \leq \sup _{c \in\left[\kappa x, \kappa^{-1} x\right]}\left(W_{t} \varphi_{t, \gamma}\right)(c),
\end{aligned}
$$

we obtain from (7.11) and (7.12) that

$$
\left(U_{t} \varphi_{t, \gamma} \omega\right)(x) \leq\left(\sup _{c \in\left[\kappa x, \kappa^{-1} x\right]}\left(W_{t} \varphi_{t, \gamma}\right)(c)\right)\left(\sup _{c \in\left[\kappa, \kappa^{-1}\right]}\left(W_{t} \varphi_{t, \gamma}\right)(c)\right)\left(U_{t} \omega\right)(x)
$$

for all $x \in(0, \infty)$. Combining (7.13) with Lemma 7.1 and Theorem 5.6(b) we see that $\left(U_{t} \varphi_{t, \gamma} \omega\right)(x)$ is bounded from above for all $x$ in some neighborhood of the point $x=1$.

To estimate $U_{t} \varphi_{t, \gamma} \omega$ from below, again let $R \in\left(0, d_{t}\right]$ and $c \in(0,1]$. From (7.6) we get

$$
\begin{aligned}
\min _{|\tau-t|=c R}\left|(\tau-t)^{\gamma}\right| & \geq\left(\left(W_{t} \varphi_{t, \gamma}\right)\left(c^{-1}\right)\right)^{-1} \max _{|\tau-t|=R}\left|(\tau-t)^{\gamma}\right|, \\
\min _{|\tau-t|=c R}\left|(\tau-t)^{-\gamma}\right| & \geq\left(\left(W_{t} \varphi_{t,-\gamma}\right)\left(c^{-1}\right)\right)^{-1} \max _{|\tau-t|=R}\left|(\tau-t)^{-\gamma}\right| \\
& =\left(\left(W_{t} \varphi_{t, \gamma}\right)(c)\right)^{-1} \max _{|\tau-t|=R}\left|(\tau-t)^{-\gamma}\right| .
\end{aligned}
$$


Taking into account (7.14) and (7.15) we obtain, for $x \in(0,1]$,

$$
\begin{aligned}
& \left(\Delta_{t}\left(\varphi_{t, \gamma}^{p} \omega^{p}, \kappa x R, x R\right)\right)^{1 / p} \\
& \geq\left(\inf _{c \in[\kappa x, x)} \min _{|\tau-t|=c R}\left|(\tau-t)^{\gamma}\right|\right)\left(\Delta_{t}\left(\omega^{p}, \kappa x R, x R\right)\right)^{1 / p} \\
& \geq\left(\inf _{c \in[\kappa x, x)}\left(\left(W_{t} \varphi_{t, \gamma}\right)\left(c^{-1}\right)\right)^{-1}\right)\left(\max _{|\tau-t|=R}\left|(\tau-t)^{\gamma}\right|\right)\left(\Delta_{t}\left(\omega^{p}, \kappa x R, x R\right)\right)^{1 / p} \\
& =\left(\sup _{c \in[\kappa x, x)}\left(W_{t} \varphi_{t, \gamma}\right)\left(c^{-1}\right)\right)^{-1}\left(\max _{|\tau-t|=R}\left|(\tau-t)^{\gamma}\right|\right)\left(\Delta_{t}\left(\omega^{p}, \kappa x R, x R\right)\right)^{1 / p}
\end{aligned}
$$

and

$$
\begin{aligned}
& \left(\Delta_{t}\left(\varphi_{t, \gamma}^{-q} \omega^{-q}, \kappa R, R\right)\right)^{1 / q} \\
& \geq\left(\inf _{c \in[\kappa, 1)} \min _{|\tau-t|=c R}\left|(\tau-t)^{-\gamma}\right|\right)\left(\Delta_{t}\left(\omega^{-q}, \kappa R, R\right)\right)^{1 / q} \\
& \geq\left(\inf _{c \in[\kappa, 1)}\left(\left(W_{t} \varphi_{t, \gamma}\right)(c)\right)^{-1}\right)\left(\max _{|\tau-t|=R}\left|(\tau-t)^{-\gamma}\right|\right)\left(\Delta_{t}\left(\omega^{-q}, \kappa R, R\right)\right)^{1 / q} \\
& =\left(\sup _{c \in[\kappa, 1)}\left(W_{t} \varphi_{t, \gamma}\right)(c)\right)^{-1}\left(\max _{|\tau-t|=R}\left|(\tau-t)^{-\gamma}\right|\right)\left(\Delta_{t}\left(\omega^{-q}, \kappa R, R\right)\right)^{1 / q} .
\end{aligned}
$$

Multiplying (7.16) and (7.17) we arrive at the estimate

$$
\begin{aligned}
& \left(\Delta_{t}\left(\varphi_{t, \gamma}^{p} \omega^{p}, \kappa x R, x R\right)\right)^{1 / p}\left(\Delta_{t}\left(\varphi_{t, \gamma}^{-q} \omega^{-q}, \kappa R, R\right)\right)^{1 / q} \\
& \geq\left(\sup _{c \in[\kappa x, x)}\left(W_{t} \varphi_{t, \gamma}\right)\left(c^{-1}\right)\right)^{-1}\left(\sup _{c \in[\kappa, 1)}\left(W_{t} \varphi_{t, \gamma}\right)(c)\right)^{-1} \\
& \quad \times\left(\Delta_{t}\left(\omega^{p}, \kappa x R, x R\right)\right)^{1 / p}\left(\Delta_{t}\left(\omega^{-q}, \kappa R, R\right)\right)^{1 / q},
\end{aligned}
$$

which implies that

$$
\left(U_{t} \varphi_{t, \gamma} \omega\right)(x) \geq\left(\sup _{c \in[\kappa x, x)}\left(W_{t} \varphi_{t, \gamma}\right)\left(c^{-1}\right)\right)^{-1}\left(\sup _{c \in[\kappa, 1)}\left(W_{t} \varphi_{t, \gamma}\right)(c)\right)^{-1}\left(U_{t} \omega\right)(x)
$$

for all $x \in(0,1]$. Similarly one can prove that

$$
\left(U_{t} \varphi_{t, \gamma} \omega\right)(x) \geq\left(\sup _{c \in[\kappa, 1)}\left(W_{t} \varphi_{t, \gamma}\right)\left(c^{-1}\right)\right)^{-1}\left(\sup _{c \in\left[\kappa x^{-1}, x^{-1}\right)}\left(W_{t} \varphi_{t, \gamma}\right)(c)\right)^{-1}\left(U_{t} \omega\right)(x)
$$

for $x \in[1, \infty)$. In the same way we derived (7.13) from (7.11) and (7.12), we obtain from (7.18) and (7.19) that

$$
\geq\left(\sup _{c \in\left[\kappa, \kappa^{-1}\right]}\left(W_{t} \varphi_{t, \gamma}\right)\left(c^{-1}\right)\right)^{-1}\left(\sup _{c \in\left[\kappa x^{-1}, \kappa^{-1} x^{-1}\right]}\left(W_{t} \varphi_{t, \gamma}\right)(c)\right)^{-1}\left(U_{t} \omega\right)(x)
$$

for all $x \in(0, \infty)$. Now Lemma 7.1 and Theorem 5.6(b) imply that $\left(U_{t} \varphi_{t, \gamma} \omega\right)(x)$ is bounded away from zero for all $x$ close enough to $x=1$.

We are left with verifying (7.4). Obviously, $\varphi_{t, \gamma} \omega=\varphi_{t, \operatorname{Re} \gamma} \psi$ with $\psi=\eta_{t}^{\operatorname{Im} \gamma} \omega$. Assume $x \in(1, \infty)$. From the definition of $U_{t}$ we see that

$$
\kappa^{|\operatorname{Re} \gamma|} x^{\operatorname{Re} \gamma}\left(U_{t} \psi\right)(x) \leq\left(U_{t} \varphi_{t, \operatorname{Re} \gamma} \psi\right)(x) \leq(1 / \kappa)^{|\operatorname{Re} \gamma|} x^{\operatorname{Re} \gamma}\left(U_{t} \psi\right)(x),
$$


whence $\beta\left(U_{t} \varphi_{t, \operatorname{Re} \gamma} \psi\right)=\operatorname{Re} \gamma+\beta\left(U_{t} \psi\right)$, which is the second equality in (7.4). The first equality can be shown analogously.

Theorem 7.3. Define

$$
\alpha_{t}^{*}(x):=\alpha\left(U_{t} \eta_{t}^{x} \omega\right), \quad \beta_{t}^{*}(x):=\beta\left(U_{t} \eta_{t}^{x} \omega\right) \quad(x \in \mathbf{R}) .
$$

Then

$$
N_{t}=\left\{\gamma \in \mathbf{C}:-\frac{1}{p}<\operatorname{Re} \gamma+\alpha_{t}^{*}(\operatorname{Im} \gamma) \leq \operatorname{Re} \gamma+\beta_{t}^{*}(\operatorname{Im} \gamma)<\frac{1}{q}\right\} .
$$

Proof. By virtue of Lemma 7.2, the equality (7.22) is equivalent to the equality

$$
N_{t}=\left\{\gamma \in \mathbf{C}:-\frac{1}{p}<\alpha\left(U_{t} \varphi_{t, \gamma} \omega\right) \leq \beta\left(U_{t} \varphi_{t, \gamma} \omega\right)<\frac{1}{q}\right\} .
$$

Suppose first that $-1 / p<\alpha\left(U_{t} \varphi_{t, \gamma} \omega\right) \leq \beta\left(U_{t} \varphi_{t, \gamma} \omega\right)<1 / q$. Then, by Theorem $6.1, B_{t}\left(\varphi_{t, \gamma} \omega\right)<\infty$. To deduce that $\varphi_{t, \gamma} \omega \in A_{p}(\Gamma)$, we have to estimate $B_{t_{0}}\left(\varphi_{t, \gamma} \omega\right)$ for $t_{0} \in \Gamma \backslash\{t\}$.

As in the proof of Theorem 7.1 of [5] we get

$$
\begin{aligned}
& \text { (7.24) } \sup _{R \geq\left|t-t_{0}\right| / 2}\left(\frac{1}{R} \int_{\Gamma\left(t_{0}, R\right)} \varphi_{t, \gamma}^{p}(\tau) \omega^{p}(\tau)|d \tau|\right)^{1 / p}\left(\frac{1}{R} \int_{\Gamma\left(t_{0}, R\right)} \varphi_{t, \gamma}^{-q}(\tau) \omega^{-q}(\tau)|d \tau|\right)^{1 / q} \\
& \quad \leq 3 B_{t}\left(\varphi_{t, \gamma} \omega\right)<\infty .
\end{aligned}
$$

So assume $0<R<\left|t-t_{0}\right| / 2$. Put

$$
R_{0}:=\left|t-t_{0}\right|-R \text { and } R_{1}:=\min \left\{\left|t-t_{0}\right|+R, d_{t}\right\} .
$$

From (7.7) and (7.8) we obtain

$$
\begin{aligned}
& \left(\frac{1}{R} \int_{\Gamma\left(t_{0}, R\right)} \varphi_{t, \gamma}^{p}(\tau) \omega^{p}(\tau)|d \tau|\right)^{1 / p} \\
& \leq\left(\sup _{x \in\left[R_{0}, R_{1}\right]|\tau-t|=x} \max _{\mid}\left|(\tau-t)^{\gamma}\right|\right)\left(\frac{1}{R} \int_{\Gamma\left(t_{0}, R\right)} \omega^{p}(\tau)|d \tau|\right)^{1 / p} \\
& \leq\left(\sup _{x \in\left[R_{0}, R_{1}\right]}\left(W_{t} \varphi_{t, \gamma}\right)\left(\frac{x}{R_{1}}\right)\right)\left(\min _{|\tau-t|=R_{1}}\left|(\tau-t)^{\gamma}\right|\right)\left(\frac{1}{R} \int_{\Gamma\left(t_{0}, R\right)} \omega^{p}(\tau)|d \tau|\right)^{1 / p}
\end{aligned}
$$

and

$$
\begin{aligned}
& \left(\frac{1}{R} \int_{\Gamma\left(t_{0}, R\right)} \varphi_{t, \gamma}^{-q}(\tau) \omega^{-q}(\tau)|d \tau|\right)^{1 / q} \\
& \leq\left(\sup _{x \in\left[R_{0}, R_{1}\right]} \max _{|\tau-t|=x}\left|(\tau-t)^{-\gamma}\right|\right)\left(\frac{1}{R} \int_{\Gamma\left(t_{0}, R\right)} \omega^{-q}(\tau)|d \tau|\right)^{1 / q} \\
& \leq\left(\sup _{x \in\left[R_{0}, R_{1}\right]}\left(W_{t} \varphi_{t, \gamma}\right)\left(\frac{R_{1}}{x}\right)\right)\left(\min _{|\tau-t|=R_{1}}\left|(\tau-t)^{-\gamma}\right|\right)\left(\frac{1}{R} \int_{\Gamma\left(t_{0}, R\right)} \omega^{-q}(\tau)|d \tau|\right)^{1 / q},
\end{aligned}
$$


whence

$$
\begin{aligned}
\text { (7.25) } & \sup \left(\frac{1}{R} \int_{\Gamma\left(t_{0}, R\right)} \varphi_{t, \gamma}^{p}(\tau) \omega^{p}(\tau)|d \tau|\right)^{1 / p}\left(\frac{1}{R} \int_{\Gamma\left(t_{0}, R\right)} \varphi_{t, \gamma}^{-q}(\tau) \omega^{-q}(\tau)|d \tau|\right)^{1 / q} \\
& \leq \sup \left(\left(\sup _{x \in\left[R_{0}, R_{1}\right]}\left(W_{t} \varphi_{t, \gamma}\right)\left(\frac{x}{R_{1}}\right)\right)\left(\sup _{x \in\left[R_{0}, R_{1}\right]}\left(W_{t} \varphi_{t, \gamma}\right)\left(\frac{R_{1}}{x}\right)\right)\right) B_{t_{0}}(\omega),
\end{aligned}
$$

the supremum over all $R$ such that $0<R<\left|t-t_{0}\right| / 2$. In the proof of Theorem 7.1 of [5] we showed that $1 / 3 \leq R_{0} / R_{1} \leq x / R_{1} \leq 1$. Thus, combining (7.24) and (7.25) we arrive at the estimate

$$
B_{t_{0}}\left(\varphi_{t, \gamma} \omega\right) \leq \max \left\{3 B_{t}\left(\varphi_{t, \gamma} \omega\right), \sup _{c \in\left[\frac{1}{3}, 1\right]}\left(W_{t} \varphi_{t, \gamma}\right)(c) \sup _{c \in[1,3]}\left(W_{t} \varphi_{t, \gamma}\right)(c) B_{t_{0}}(\omega)\right\} .
$$

Since $W_{t} \varphi_{t, \gamma}$ is regular (Lemma 7.1) and $\sup _{t_{0}} B_{t_{0}}(\omega)<\infty$ (because $\omega \in A_{p}(\Gamma)$ ), it follows that $\sup _{t_{0}} B_{t_{0}}\left(\varphi_{t, \gamma} \omega\right)<\infty$. Thus, $\varphi_{t, \gamma} \omega \in A_{p}(\Gamma)$.

Conversely, if $\varphi_{t, \gamma} \omega \in A_{p}(\Gamma)$, then $U \varphi_{t, \gamma} \omega$ and $U_{t} \varphi_{t, \gamma} \omega$ are regular submultiplicative functions due to Theorem 5.6(b), and

$$
-1 / p<\alpha\left(U \varphi_{t, \gamma} \omega\right) \leq \beta\left(U \varphi_{t, \gamma} \omega\right)<1 / q
$$

due to Theorem 6.2. We have $\log \left(U_{t} \varphi_{t, \gamma} \omega\right)(x) \leq \log \left(U \varphi_{t, \gamma} \omega\right)(x)$ for all $x \in(0, \infty)$. If $x \in(0,1)$, then $\log x<0$ and consequently,

$$
\alpha\left(U_{t} \varphi_{t, \gamma} \omega\right)=\lim _{x \rightarrow 0} \frac{\log \left(U_{t} \varphi_{t, \gamma} \omega\right)(x)}{\log x} \geq \lim _{x \rightarrow 0} \frac{\log \left(U \varphi_{t, \gamma} \omega\right)(x)}{\log x}=\alpha\left(U \varphi_{t, \gamma} \omega\right) .
$$

Thus, (7.26) gives $\alpha\left(U_{t} \varphi_{t, \gamma} \omega\right)>-1 / p$. In the same manner one can show that $\beta\left(U_{t} \varphi_{t, \gamma} \omega\right)<1 / q$.

Clearly, $\alpha_{t}{ }^{*}(x) \leq \beta_{t}{ }^{*}(x)$ for all $x \in \mathbf{R}$. Consequently, Theorems 4.4 and 7.3 imply that Theorem 3.1 holds with $\alpha_{t}{ }^{*}, \beta_{t}{ }^{*}$ given by (7.21) in the place of $\alpha_{t}, \beta_{t}$.

\section{THE INDICATOR FUNCTIONS}

The purpose of this section is to prove that Theorems 3.1 and 3.5 hold with the functions $\alpha_{t}, \beta_{t}$ defined by (3.4).

Lemma 8.1. The functions $V_{t} \varphi_{t, \gamma} \omega$ and $V_{t}^{0} \varphi_{t, \gamma} \omega$ are regular and submultiplicative for every $\gamma \in \mathbf{C}$. We have

$$
\begin{aligned}
& \alpha\left(U_{t} \varphi_{t, \gamma} \omega\right) \leq \alpha\left(V_{t} \varphi_{t, \gamma} \omega\right)=\alpha\left(V_{t}^{0} \varphi_{t, \gamma} \omega\right) \\
& \beta\left(U_{t} \varphi_{t, \gamma} \omega\right) \geq \beta\left(V_{t} \varphi_{t, \gamma} \omega\right)=\beta\left(V_{t}^{0} \varphi_{t, \gamma} \omega\right)
\end{aligned}
$$

for all $\gamma \in \mathbf{C}$. In particular,

$$
\alpha_{t}{ }^{*}(x) \leq \alpha_{t}(x) \leq \beta_{t}(x) \leq \beta_{t}^{*}(x) \text { for all } x \in \mathbf{R} .
$$

Moreover,

$$
\alpha\left(V_{t}^{0} \varphi_{t, \gamma} \omega\right)=\operatorname{Re} \gamma+\alpha\left(V_{t}^{0} \eta_{t}^{\operatorname{Im} \gamma} \omega\right), \quad \beta\left(V_{t}^{0} \varphi_{t, \gamma} \omega\right)=\operatorname{Re} \gamma+\beta\left(V_{t}^{0} \eta_{t}^{\operatorname{Im} \gamma} \omega\right)
$$

Proof. Combining Lemma 7.1 and Theorem 6.3 we conclude that $\varphi_{t, \gamma} \in A_{p}(\Gamma)$ whenever $|\gamma|$ is sufficiently small. For these $\gamma$ we have $\log \varphi_{t, \gamma} \in B M O(\Gamma)$ due to Lemma 5.3, and since

$$
\log \varphi_{t, \gamma}(\tau)=\operatorname{Re} \gamma \log |\tau-t|-\operatorname{Im} \gamma \arg (\tau-t),
$$


it follows that $\log |\tau-t|$ and $\arg (\tau-t)(\tau \in \Gamma \backslash\{t\})$ are functions in $B M O(\Gamma)$. Thus, from (8.5) we infer that $\log \varphi_{t, \gamma} \in B M O(\Gamma)$ for all $\gamma \in \mathbf{C}$. Again having recourse to Lemma 5.3 we deduce that $\log \varphi_{t, \gamma} \omega=\log \varphi_{t, \gamma}+\log \omega \in B M O(\Gamma)$ and consequently, by Theorem 5.6(a), $V_{t} \varphi_{t, \gamma} \omega$ and $V_{t}^{0} \varphi_{t, \gamma} \omega$ are regular and submultiplicative.

To show (8.1)-(8.4), notice first that, by Hölder's inequality,

$$
\begin{aligned}
& \left(\frac{1}{|I|} \int_{I} \psi(\tau)^{\varepsilon p}|d \tau|\right)^{1 / p} \leq\left(\frac{1}{|I|} \int_{I} \psi(\tau)^{p}|d \tau|\right)^{\varepsilon / p}\left(\frac{1}{|I|} \int_{I}|d \tau|\right)^{(1-\varepsilon) / p}, \\
& \left(\frac{1}{|I|} \int_{I} \psi(\tau)^{-\varepsilon q}|d \tau|\right)^{1 / q} \leq\left(\frac{1}{|I|} \int_{I} \psi(\tau)^{-q}|d \tau|\right)^{\varepsilon / q}\left(\frac{1}{|I|} \int_{I}|d \tau|\right)^{(1-\varepsilon) / q}
\end{aligned}
$$

for every measurable subset $I$ of $\Gamma$ and every $\varepsilon \in(0,1)$. This easily implies that

$$
\left(U_{t} \varphi_{t, \varepsilon \gamma} \omega^{\varepsilon}\right)(x) \leq\left(\left(U_{t} \varphi_{t, \gamma} \omega\right)(x)\right)^{\varepsilon} \text { for all } x \in(0, \infty)
$$

and thus,

$$
\varepsilon \alpha\left(U_{t} \varphi_{t, \gamma} \omega\right) \leq \alpha\left(U_{t} \varphi_{t, \varepsilon \gamma} \omega^{\varepsilon}\right) \leq \beta\left(U_{t} \varphi_{t, \varepsilon \gamma} \omega^{\varepsilon}\right) \leq \varepsilon \beta\left(U_{t} \varphi_{t, \gamma} \omega\right) .
$$

From (8.6) and (7.23) we obtain that

$$
\varphi_{t, \varepsilon \gamma} \omega^{\varepsilon} \in A_{p}(\Gamma), \quad \eta_{t}^{\varepsilon \operatorname{Im} \gamma} \omega^{\varepsilon}=\varphi_{t, i \varepsilon \operatorname{Im} \gamma} \omega^{\varepsilon} \in A_{p}(\Gamma)
$$

for all sufficiently small $\varepsilon \in(0,1)$, for $\varepsilon \in\left(0, \varepsilon_{0}\right)$ say.

Fix $\varepsilon \in\left(0, \varepsilon_{0}\right)$. By the definition of $V_{t}$ and $V_{t}^{0}$,

$$
\alpha\left(V_{t}^{0} \varphi_{t, \gamma} \omega\right)=\frac{1}{\varepsilon} \alpha\left(V_{t}^{0} \varphi_{t, \varepsilon \gamma} \omega^{\varepsilon}\right), \quad \alpha\left(V_{t} \varphi_{t, \gamma} \omega\right)=\frac{1}{\varepsilon} \alpha\left(V_{t} \varphi_{t, \varepsilon \gamma} \omega^{\varepsilon}\right) .
$$

Since $\alpha\left(V_{t}^{0} \varphi_{t, \varepsilon \gamma} \omega^{\varepsilon}\right)=\alpha\left(V_{t} \varphi_{t, \varepsilon \gamma} \omega^{\varepsilon}\right)$ due to Lemma 5.9, we obtain from (8.7) that $\alpha\left(V_{t}^{0} \varphi_{t, \gamma} \omega\right)=\alpha\left(V_{t} \varphi_{t, \gamma} \omega\right)$. Further, from (8.7) and Lemmas 5.8 and 5.9 we also deduce that

$$
\alpha\left(V_{t}^{0} \varphi_{t, \gamma} \omega\right)=\frac{1}{\varepsilon} \alpha\left(U_{t} \varphi_{t, \varepsilon \gamma} \omega^{\varepsilon}\right)
$$

Analogously,

$$
\alpha\left(V_{t}^{0} \eta_{t}^{\operatorname{Im} \gamma} \omega\right)=\frac{1}{\varepsilon} \alpha\left(U_{t} \eta_{t}^{\varepsilon \operatorname{Im} \gamma} \omega^{\varepsilon}\right)
$$

Combining (8.8), (8.9), and (7.4), we get the first equality of (8.4). The second equality of (8.7) and Lemma 5.8 imply that

$$
\alpha\left(V_{t} \varphi_{t, \gamma} \omega\right)=\frac{1}{\varepsilon} \alpha\left(U_{t} \varphi_{t, \varepsilon \gamma} \omega^{\varepsilon}\right)
$$

From (8.10) and (8.6) we get $\alpha\left(U_{t} \varphi_{t, \gamma} \omega\right) \leq \alpha\left(V_{t} \varphi_{t, \gamma} \omega\right)$, which completes the proof of (8.1). The second equality in (8.4) and assertion (8.2) can be shown analogously. Clearly, (8.3) results from (8.1) and (8.2) with $\gamma=i x$.

Thus, the functions (3.4) are well-defined for every $x \in \mathbf{R}$. Clearly, $\alpha_{t}(x) \leq \beta_{t}(x)$ for all $x$.

Lemma 8.2. The functions $\alpha_{t}{ }^{*}$ and $\alpha_{t}$ are concave on $\mathbf{R}$; the functions $\beta_{t}{ }^{*}$ and $\beta_{t}$ are convex on $\mathbf{R}$. These functions are in particular continuous on all of $\mathbf{R}$. 
Proof. Since $\int f^{\theta} g^{1-\theta} d \mu \leq\left(\int f d \mu\right)^{\theta}\left(\int g d \mu\right)^{1-\theta}$ for $\theta \in(0,1)$ by Hölder's inequality, we get

$$
\begin{aligned}
& \left(\Delta_{t}\left(\eta_{t}^{p(x \theta+y(1-\theta))} \omega^{p}, \kappa \xi R, \xi R\right)\right)^{1 / p} \\
& \leq\left(\Delta_{t}\left(\eta_{t}^{p x} \omega^{p}, \kappa \xi R, \xi R\right)\right)^{\theta / p}\left(\Delta_{t}\left(\eta_{t}^{p y} \omega^{p}, \kappa \xi R, \xi R\right)\right)^{(1-\theta) / p} \\
& \left(\Delta_{t}\left(\eta_{t}^{-q(x \theta+y(1-\theta))} \omega^{-q}, \kappa R, R\right)\right)^{1 / q} \\
& \leq\left(\Delta_{t}\left(\eta_{t}{ }^{-q x} \omega^{-q}, \kappa R, R\right)\right)^{\theta / q}\left(\Delta_{t}\left(\eta_{t}^{-q y} \omega^{-q}, \kappa R, R\right)\right)^{(1-\theta) / q} .
\end{aligned}
$$

Multiplying these two inequalities and taking $\sup _{R>0}$ we arrive at the inequality

$$
\left(U_{t} \eta_{t}{ }^{x \theta+y(1-\theta)} \omega\right)(\xi) \leq\left[\left(U_{t} \eta_{t}{ }^{x} \omega\right)(\xi)\right]^{\theta}\left[\left(U_{t} \eta_{t}{ }^{y} \omega\right)(\xi)\right]^{1-\theta}
$$

for $\xi \in(0,1)$, which shows that $\alpha_{t}{ }^{*}$ is concave. The convexity of $\beta_{t}{ }^{*}$ can be verified analogously.

Obviously,

$$
\begin{aligned}
& \frac{\exp \left(\Delta_{t}\left(\log \eta_{t}^{x \theta+y(1-\theta)} \omega, 0, \xi R\right)\right)}{\exp \left(\Delta_{t}\left(\log \eta_{t} x \theta+y(1-\theta) \omega, 0, R\right)\right)} \\
& =\left(\frac{\exp \left(\Delta_{t}\left(\log \eta_{t}^{x} \omega, 0, \xi R\right)\right)}{\exp \left(\Delta_{t}\left(\log \eta_{t}^{x} \omega, 0, R\right)\right)}\right)^{\theta}\left(\frac{\exp \left(\Delta_{t}\left(\log \eta_{t}^{y} \omega, 0, \xi R\right)\right)}{\exp \left(\Delta_{t}\left(\log \eta_{t}^{y} \omega, 0, R\right)\right)}\right)^{1-\theta}
\end{aligned}
$$

and taking $\lim \sup _{R \rightarrow 0}$ we obtain

$$
\left(V_{t}^{0} \eta_{t}^{x \theta+y(1-\theta)} \omega\right)(\xi) \leq\left[\left(V_{t}^{0} \eta_{t}^{x} \omega\right)(\xi)\right]^{\theta}\left[\left(V_{t}^{0} \eta_{t}^{y} \omega\right)(\xi)\right]^{1-\theta}
$$

for $\xi \in(0,1)$, implying that $\alpha_{t}$ is concave. In the same way one can prove that $\beta_{t}$ is convex. As already mentioned in Section 3, convexity/concavity implies continuity.

Proof of Theorem 3.5. From Theorem 7.3 we infer that $N_{t}$ has the form (7.22). Put $c(x)=\beta_{t}^{*}(x)-\alpha_{t}^{*}(x)$. The function $c$ is convex due to Lemma 8.2, and $c(0)=\beta\left(U_{t} \omega\right)-\alpha\left(U_{t} \omega\right)<1$ by Theorem 7.3, because $\omega \in A_{p}(\Gamma)$ and hence $0 \in N_{t}$. Consequently, either there exists a unique $x_{t}^{+}>0$ such that $c\left(x_{t}^{+}\right)=1$ or we have $0 \leq c(x)<1$ for all $x>0$, in which case we put $x_{t}^{+}:=+\infty$. Analogously, either $c\left(x_{t}^{-}\right)=1$ for some uniquely determined $x_{t}^{-}<0$ or $0 \leq c(x)<1$ for all $x<0$, in which case we define $x_{t}^{-}:=-\infty$.

Fix $x \in\left(x_{t}^{-}, x_{t}^{+}\right)$. Then $0 \leq \beta_{t}{ }^{*}(x)-\alpha_{t}{ }^{*}(x)<1$ by the convexity of $c$. Hence, there is a real number $\mu$ such that

$$
-1 / p<\mu+\alpha_{t}{ }^{*}(x) \leq \mu+\beta_{t}^{*}(x)<1 / q,
$$

and Theorem 7.3 implies that $\mu+i x \in N_{t}$, i.e. that $\varphi_{t, \mu+i x} \omega \in A_{p}(\Gamma)$. Lemmas 5.8 and 5.9 give

$$
\alpha\left(V_{t}^{0} \varphi_{t, \mu+i x} \omega\right)=\alpha\left(U_{t} \varphi_{t, \mu+i x} \omega\right)
$$

and by virtue of (8.4) and (7.4) the latter equality may be written in the form

$$
\mu+\alpha\left(V_{t}^{0} \eta_{t}^{x} \omega\right)=\mu+\alpha\left(U_{t} \eta_{t}^{x} \omega\right)
$$

which proves that $\alpha_{t}(x)=\alpha_{t}{ }^{*}(x)$. Analogously one can show that $\beta_{t}(x)=\beta_{t}{ }^{*}(x)$ whenever $x_{t}^{-}<x<x_{t}^{+}$. Since $\alpha_{t}, \alpha_{t}{ }^{*}, \beta_{t}, \beta_{t}{ }^{*}$ are continuous (Lemma 8.2), we see that $\alpha_{t}=\alpha_{t}{ }^{*}$ and $\beta_{t}=\beta_{t}{ }^{*}$ on $\left[x_{t}^{-}, x_{t}^{+}\right]$.

If $x_{t}^{-}=-\infty$ and $x_{t}^{+}=+\infty$, the proof is complete. Suppose $x_{t}^{+}<+\infty$. Denote the set on the right of (4.6) by $\tilde{N}_{t}$. Since

$$
\beta_{t}\left(x_{t}^{+}\right)-\alpha_{t}\left(x_{t}^{+}\right)=\beta_{t}^{*}\left(x_{t}^{+}\right)-\alpha_{t}{ }^{*}\left(x_{t}^{+}\right)=1
$$


and hence, by the convexity of $\beta_{t}{ }^{*}-\alpha_{t}{ }^{*}$ and $\beta_{t}-\alpha_{t}$,

$$
\beta_{t}{ }^{*}(\operatorname{Im} \gamma)-\alpha_{t}{ }^{*}(\operatorname{Im} \gamma) \geq 1, \quad \beta_{t}(\operatorname{Im} \gamma)-\alpha_{t}(\operatorname{Im} \gamma) \geq 1 \text { for } \operatorname{Im} \gamma>x_{t}^{+},
$$

it follows that

$$
\begin{aligned}
& N_{t}=\left\{\gamma \in \mathbf{C}: \operatorname{Im} \gamma \leq x_{t}^{+},-\frac{1}{p}<\operatorname{Re} \gamma+\alpha_{t}{ }^{*}(\operatorname{Im} \gamma) \leq \operatorname{Re} \gamma+\beta_{t}^{*}(\operatorname{Im} \gamma)<\frac{1}{q}\right\}, \\
& \tilde{N}_{t}=\left\{\gamma \in \mathbf{C}: \operatorname{Im} \gamma \leq x_{t}^{+},-\frac{1}{p}<\operatorname{Re} \gamma+\alpha_{t}(\operatorname{Im} \gamma) \leq \operatorname{Re} \gamma+\beta_{t}(\operatorname{Im} \gamma)<\frac{1}{q}\right\} .
\end{aligned}
$$

If $x_{t}^{-}=-\infty$, this completes the proof. In case $x_{t}^{-}>-\infty$ we analogously see that $N_{t}$ and $\tilde{N}_{t}$ are the sets

$$
\begin{aligned}
& \left\{\gamma \in \mathbf{C}: x_{t}^{-} \leq \operatorname{Im} \gamma \leq x_{t}^{+},-\frac{1}{p}<\operatorname{Re} \gamma+\alpha_{t}{ }^{*}(\operatorname{Im} \gamma) \leq \operatorname{Re} \gamma+\beta_{t}^{*}(\operatorname{Im} \gamma)<\frac{1}{q}\right\}, \\
& \left\{\gamma \in \mathbf{C}: x_{t}^{-} \leq \operatorname{Im} \gamma \leq x_{t}^{+},-\frac{1}{p}<\operatorname{Re} \gamma+\alpha_{t}(\operatorname{Im} \gamma) \leq \operatorname{Re} \gamma+\beta_{t}(\operatorname{Im} \gamma)<\frac{1}{q}\right\},
\end{aligned}
$$

respectively. As $\alpha_{t}=\alpha_{t}{ }^{*}$ and $\beta_{t}=\beta_{t}{ }^{*}$ on $\left[x_{t}^{-}, x_{t}^{+}\right]$, we arrive at the desired equality $N_{t}=\tilde{N}_{t}$.

Combining Theorems 4.4 and 3.5 we obtain Theorem 3.1 with $\alpha_{t}, \beta_{t}$ given by

\section{ThE SHAPE OF THE INDICATOR FUnCTIONS}

In this section we prove Theorems 3.2 and 3.3 .

Lemma 9.1. For every $\gamma \in \mathbf{C}$ we have

$$
\begin{aligned}
& \alpha\left(U_{t} \omega\right)+\alpha\left(W_{t} \varphi_{t, \gamma}\right) \leq \alpha\left(U_{t} \varphi_{t, \gamma} \omega\right) \leq \alpha\left(U_{t} \omega\right)+\beta\left(W_{t} \varphi_{t, \gamma}\right), \\
& \beta\left(U_{t} \omega\right)+\alpha\left(W_{t} \varphi_{t, \gamma}\right) \leq \beta\left(U_{t} \varphi_{t, \gamma} \omega\right) \leq \beta\left(U_{t} \omega\right)+\beta\left(W_{t} \varphi_{t, \gamma}\right) .
\end{aligned}
$$

Proof. If $x \in(0,1)$, then $\log x<0$ and hence, by (7.13),

$$
\begin{aligned}
& \frac{\log \left(U_{t} \varphi_{t, \gamma} \omega\right)(x)}{\log x} \\
& \geq \frac{C}{\log x}+\frac{\log \sup \left\{\left(W_{t} \varphi_{t, \gamma}\right)(c): c \in\left[\kappa x, \kappa^{-1} x\right]\right\}}{\log x}+\frac{\log \left(U_{t} \omega\right)(x)}{\log x}
\end{aligned}
$$

with $C:=\log \sup \left\{\left(W_{t} \varphi_{t, \gamma}\right)(c): c \in\left[\kappa, \kappa^{-1}\right]\right\}$. Let $\varepsilon>0$. Theorem 5.1(c) shows that

$$
\begin{aligned}
\sup _{c \in\left[\kappa x, \kappa^{-1} x\right]}\left(W_{t} \varphi_{t, \gamma}\right)(c) & \leq \max \left\{\left(\kappa^{-1} x\right)^{\alpha\left(W_{t} \varphi_{t, \gamma}\right)-\varepsilon}, \quad(\kappa x)^{\alpha\left(W_{t} \varphi_{t, \gamma}\right)-\varepsilon}\right\} \\
& \leq \kappa^{-\left|\alpha\left(W_{t} \varphi_{t, \gamma}\right)-\varepsilon\right|} x^{\alpha\left(W_{t} \varphi_{t, \gamma}\right)-\varepsilon}
\end{aligned}
$$

for all sufficiently small $x>0$. For these $x$, the second term on the right of (9.3) is at least

$$
\left(\left(\alpha\left(W_{t} \varphi_{t, \gamma}\right)-\varepsilon\right) \log x-\left|\alpha\left(W_{t} \varphi_{t, \gamma}\right)-\varepsilon\right| \log \kappa\right) / \log x
$$

and therefore passage to the limit $x \rightarrow 0$ in (9.3) gives

$$
\alpha\left(U_{t} \varphi_{t, \gamma} \omega\right) \geq \alpha\left(W_{t} \varphi_{t, \gamma}\right)-\varepsilon+\alpha\left(U_{t} \omega\right) .
$$

As $\varepsilon>0$ was arbitrary, we get the first inequality of (9.1). 
Similarly, for $x \in(0,1)$ the inequality $(7.20)$ tells us that

$$
\begin{aligned}
& \frac{\log \left(U_{t} \varphi_{t, \gamma} \omega\right)(x)}{\log x} \\
& \leq-\frac{C}{\log x}+\frac{\log \sup \left\{\left(W_{t} \varphi_{t, \gamma}\right)(c): c \in\left[\kappa x^{-1}, \kappa^{-1} x^{-1}\right]\right\}}{\log (1 / x)}+\frac{\log \left(U_{t} \omega\right)(x)}{\log x}
\end{aligned}
$$

with $C$ as above. Again pick any $\varepsilon>0$. Then, by Theorem 5.1(c),

$$
\begin{aligned}
\sup _{c \in\left[\kappa x^{-1}, \kappa^{-1} x^{-1}\right]}\left(W_{t} \varphi_{t, \gamma}\right)(c) & \leq \max \left\{1 /(\kappa x)^{\beta\left(W_{t} \varphi_{t, \gamma}\right)+\varepsilon},(\kappa / x)^{\beta\left(W_{t} \varphi_{t, \gamma}\right)+\varepsilon}\right\} \\
& \leq \kappa^{-\left|\beta\left(W_{t} \varphi_{t, \gamma}\right)+\varepsilon\right|}(1 / x)^{\beta\left(W_{t} \varphi_{t, \gamma}\right)+\varepsilon}
\end{aligned}
$$

for all $x$ small enough. For these $x$ the second term on the right of (9.4) is at most

$$
\left(\left(\beta\left(W_{t} \varphi_{t, \gamma}\right)+\varepsilon\right) \log (1 / x)-\left|\beta\left(W_{t} \varphi_{t, \gamma}\right)+\varepsilon\right| \log \kappa\right) / \log (1 / x),
$$

and passing to the limit $x \rightarrow 0$ in (9.4) we arrive at the inequality

$$
\alpha\left(U_{t} \varphi_{t, \gamma} \omega\right) \leq \beta\left(W_{t} \varphi_{t, \gamma}\right)+\varepsilon+\alpha\left(U_{t} \omega\right) .
$$

Since $\varepsilon>0$ may be chosen as small as desired, we get the second inequality of (9.1).

Employing (7.13) and (7.20) for $x \in(1, \infty)$, we analogously obtain (9.2).

Lemma 9.2. For every $\gamma \in \mathbf{C}$,

$$
\begin{aligned}
& \alpha\left(W_{t} \varphi_{t, \gamma}\right)=\alpha\left(U_{t} \varphi_{t, \gamma}\right)=\alpha\left(V_{t} \varphi_{t, \gamma}\right)=\alpha\left(V_{t}^{0} \varphi_{t, \gamma}\right), \\
& \beta\left(W_{t} \varphi_{t, \gamma}\right)=\beta\left(U_{t} \varphi_{t, \gamma}\right)=\beta\left(V_{t} \varphi_{t, \gamma}\right)=\beta\left(V_{t}^{0} \varphi_{t, \gamma}\right) .
\end{aligned}
$$

Proof. The inequalities (9.1), (9.2), (8.1), (8.2) with $\omega=1$ give

$$
\begin{aligned}
& \alpha\left(W_{t} \varphi_{t, \gamma}\right) \leq \alpha\left(U_{t} \varphi_{t, \gamma}\right) \leq \alpha\left(V_{t} \varphi_{t, \gamma}\right)=\alpha\left(V_{t}^{0} \varphi_{t, \gamma}\right) \\
& \leq \beta\left(V_{t}^{0} \varphi_{t, \gamma}\right)=\beta\left(V_{t} \varphi_{t, \gamma}\right) \leq \beta\left(U_{t} \varphi_{t, \gamma}\right) \leq \beta\left(W_{t} \varphi_{t, \gamma}\right) .
\end{aligned}
$$

For $\varepsilon \neq 0$ we have

$$
\alpha\left(V_{t} \varphi_{t, \gamma}\right)=\frac{1}{\varepsilon} \alpha\left(V_{t} \varphi_{t, \varepsilon \gamma}\right), \beta\left(V_{t} \varphi_{t, \gamma}\right)=\frac{1}{\varepsilon} \beta\left(V_{t} \varphi_{t, \varepsilon \gamma}\right)
$$

by the definition of $V_{t}$, and

$$
\alpha\left(W_{t} \varphi_{t, \gamma}\right)=\frac{1}{\varepsilon} \alpha\left(W_{t} \varphi_{t, \varepsilon \gamma}\right), \beta\left(W_{t} \varphi_{t, \gamma}\right)=\frac{1}{\varepsilon} \beta\left(W_{t} \varphi_{t, \varepsilon \gamma}\right)
$$

due to Lemma 7.1. If $\varepsilon>0$ is small enough, then (9.8) implies that

$$
-\frac{1}{p}<\alpha\left(W_{t} \varphi_{t, \varepsilon \gamma}\right) \leq \beta\left(W_{t} \varphi_{t, \varepsilon \gamma}\right)<\frac{1}{q}
$$

and hence, by Lemma 7.1 and Theorem $6.3, \varphi_{t, \varepsilon \gamma} \in A_{p}(\Gamma)$. Replacing in (9.6) $\gamma$ by $\varepsilon \gamma$, we obtain from (9.9) that

$$
-\frac{1}{p}<\alpha\left(W_{t} \varphi_{t, \varepsilon \gamma}\right) \leq \alpha\left(U_{t} \varphi_{t, \varepsilon \gamma}\right) \leq \beta\left(U_{t} \varphi_{t, \varepsilon \gamma}\right) \leq \beta\left(W_{t} \varphi_{t, \varepsilon \gamma}\right)<\frac{1}{q} .
$$

Assume $\alpha\left(W_{t} \varphi_{t, \varepsilon \gamma}\right)<\alpha\left(U_{t} \varphi_{t, \varepsilon \gamma}\right)$. Then there is a $\mu<0$ such that

$$
\begin{aligned}
& \mu+\alpha\left(W_{t} \varphi_{t, \varepsilon \gamma}\right)<-\frac{1}{p}<\mu+\alpha\left(U_{t} \varphi_{t, \varepsilon \gamma}\right) \\
& \leq \mu+\beta\left(U_{t} \varphi_{t, \varepsilon \gamma}\right) \leq \mu+\beta\left(W_{t} \varphi_{t, \varepsilon \gamma}\right)<\frac{1}{q} .
\end{aligned}
$$


From Lemma 7.2 we deduce that

$$
\mu+\alpha\left(U_{t} \varphi_{t, \varepsilon \gamma}\right)=\alpha\left(U_{t} \varphi_{t, \mu+\varepsilon \gamma}\right), \quad \mu+\beta\left(U_{t} \varphi_{t, \varepsilon \gamma}\right)=\beta\left(U_{t} \varphi_{t, \mu+\varepsilon \gamma}\right)
$$

and Lemma 7.1 shows that

$$
\mu+\alpha\left(W_{t} \varphi_{t, \varepsilon \gamma}\right)=\alpha\left(W_{t} \varphi_{t, \mu+\varepsilon \gamma}\right), \quad \mu+\beta\left(W_{t} \varphi_{t, \varepsilon \gamma}\right)=\beta\left(W_{t} \varphi_{t, \mu+\varepsilon \gamma}\right) .
$$

Thus, (9.10) may be rewritten in the form

$$
\alpha\left(W_{t} \varphi_{t, \mu+\varepsilon \gamma}\right)<-\frac{1}{p}<\alpha\left(U_{t} \varphi_{t, \mu+\varepsilon \gamma}\right) \leq \beta\left(U_{t} \varphi_{t, \mu+\varepsilon \gamma}\right) \leq \beta\left(W_{t} \varphi_{t, \mu+\varepsilon \gamma}\right)<\frac{1}{q} .
$$

But (9.11) in conjunction with Lemma 7.1 and Theorem 6.3 implies that $\varphi_{t, \mu+\varepsilon \gamma}$ is not in $A_{p}(\Gamma)$, while (9.11) together with (7.23) yields that $\varphi_{t, \mu+\varepsilon \gamma} \in A_{p}(\Gamma)$. This contradiction shows that $\alpha\left(W_{t} \varphi_{t, \varepsilon \gamma}\right)=\alpha\left(U_{t} \varphi_{t, \varepsilon \gamma}\right)$.

Since $\varphi_{t, \varepsilon \gamma} \in A_{p}(\Gamma)$, we obtain from Lemma 5.8 that $\alpha\left(U_{t} \varphi_{t, \varepsilon \gamma}\right)=\alpha\left(V_{t} \varphi_{t, \varepsilon \gamma}\right)$. In summary,

$$
\alpha\left(W_{t} \varphi_{t, \varepsilon \gamma}\right)=\alpha\left(U_{t} \varphi_{t, \varepsilon \gamma}\right)=\alpha\left(V_{t} \varphi_{t, \varepsilon \gamma}\right) .
$$

Now (9.7) and (9.8) give that

$$
\alpha\left(W_{t} \varphi_{t, \gamma}\right)=\alpha\left(V_{t} \varphi_{t, \gamma}\right)
$$

and hence, by (9.6), we arrive at (9.4). The equalities (9.5) can be proved in the same manner.

Lemma 9.3. For every $\gamma \in \mathbf{C}$,

$$
\begin{aligned}
& \alpha\left(V_{t}^{0} \omega\right)+\alpha\left(W_{t} \varphi_{t, \gamma}\right) \leq \alpha\left(V_{t}^{0} \varphi_{t, \gamma} \omega\right) \leq \beta\left(V_{t}^{0} \omega\right)+\alpha\left(W_{t} \varphi_{t, \gamma}\right), \\
& \alpha\left(V_{t}^{0} \omega\right)+\beta\left(W_{t} \varphi_{t, \gamma}\right) \leq \beta\left(V_{t}^{0} \varphi_{t, \gamma} \omega\right) \leq \beta\left(V_{t}^{0} \omega\right)+\beta\left(W_{t} \varphi_{t, \gamma}\right) .
\end{aligned}
$$

Proof. Fix $x \in(0,1)$. Then $\log \left(V_{t} \varphi_{t, \gamma} \omega\right)(x)$ equals

$$
\sup _{R>0}\left[\Delta_{t}\left(\log \varphi_{t, \gamma}, 0, x R\right)-\Delta_{t}\left(\log \varphi_{t, \gamma}, 0, R\right)+\Delta_{t}(\log \omega, 0, x R)-\Delta_{t}(\log \omega, 0, R)\right] .
$$

For every $\varepsilon>0$, there is an $R_{0} \in\left(0, d_{t}\right]$ such that

$$
\begin{aligned}
& \Delta_{t}\left(\log \varphi_{t, \gamma}, 0, x R_{0}\right)-\Delta_{t}\left(\log \varphi_{t, \gamma}, 0, R_{0}\right) \\
& \geq \sup _{R>0}\left[\Delta_{t}\left(\log \varphi_{t, \gamma}, 0, x R\right)-\Delta_{t}\left(\log \varphi_{t, \gamma}, 0, R\right)\right]-\varepsilon=\log \left(V_{t} \varphi_{t, \gamma}\right)(x)-\varepsilon .
\end{aligned}
$$

On the other hand,

$$
\begin{aligned}
& \Delta_{t}\left(\log \omega, 0, x R_{0}\right)-\Delta_{t}\left(\log \omega, 0, R_{0}\right) \\
& \geq-\sup _{R>0}\left[\Delta_{t}(\log \omega, 0, R)-\Delta_{t}(\log \omega, 0, x R)\right]=-\log \left(V_{t} \omega\right)\left(x^{-1}\right) .
\end{aligned}
$$

Thus, for every $x \in(0,1)$,

$$
\log \left(V_{t} \varphi_{t, \gamma} \omega\right)(x) \geq \log \left(V_{t} \varphi_{t, \gamma}\right)(x)-\log \left(V_{t} \omega\right)\left(x^{-1}\right)-\varepsilon .
$$

From (9.14) it is clear that

$$
\log \left(V_{t} \varphi_{t, \gamma}\right)(x)+\log \left(V_{t} \omega\right)(x) \geq \log \left(V_{t} \varphi_{t, \gamma} \omega\right)(x) .
$$

Dividing (9.15) and (9.16) by $\log x<0$ and passing to the limit $x \rightarrow 0$ we obtain the inequalities

$$
\alpha\left(V_{t} \varphi_{t, \gamma}\right)+\alpha\left(V_{t} \omega\right) \leq \alpha\left(V_{t} \varphi_{t, \gamma} \omega\right) \leq \alpha\left(V_{t} \varphi_{t, \gamma}\right)+\beta\left(V_{t} \omega\right) .
$$


Since $\alpha\left(V_{t} \omega\right)=\alpha\left(V_{t}^{0} \omega\right)$ and $\beta\left(V_{t} \omega\right)=\beta\left(V_{t}^{0} \omega\right)$ by Lemma 5.9, $\alpha\left(V_{t} \varphi_{t, \gamma} \omega\right)=$ $\alpha\left(V_{t}^{0} \varphi_{t, \gamma} \omega\right)$ by Lemma 8.1, and $\alpha\left(V_{t} \varphi_{t, \gamma}\right)=\alpha\left(W_{t} \varphi_{t, \gamma}\right)$ by Lemma 9.2 , we get (9.12) from (9.17). The inequalities (9.13) can be shown analogously.

Proof of Theorem 3.2. Property (a) results from Lemma 8.1 and Theorem 5.1(a), property (b) is a consequence of Theorem 3.5 and the fact that $N_{t}$ contains the origin, property (c) is implied by Lemma 8.2, and property (e) was obtained in the end of Section 8 . We are thus left with proving property $(\mathrm{d})$.

Define $\delta_{t}^{-}$and $\delta_{t}^{+}$by $(7.3)$, i.e. $\delta_{t}^{-}:=\alpha\left(W_{t} \eta_{t}\right), \delta_{t}^{+}:=\beta\left(W_{t} \eta_{t}\right)$. Fix $x>0$. From (9.13) we infer that

$$
\alpha\left(V_{t}^{0} \omega\right)+\beta\left(W_{t} \varphi_{t, i x}\right) \leq \beta\left(V_{t}^{0} \varphi_{t, i x} \omega\right) \leq \beta\left(V_{t}^{0} \omega\right)+\beta\left(W_{t} \varphi_{t, i x}\right) .
$$

Since $\delta_{t}^{-} \leq \delta_{t}^{+}$and $x>0$, equality (7.2) shows that $\beta\left(W_{t} \varphi_{t, i x}\right)=\delta_{t}^{+} x$, and since $\beta\left(V_{t}^{0} \varphi_{t, i x} \omega\right)=\beta_{t}(x)$ by the definition of $\beta_{t}$, we obtain that

$$
\alpha\left(V_{t}^{0} \omega\right)+\delta_{t}^{+} x \leq \beta_{t}(x) \leq \beta\left(V_{t}^{0} \omega\right)+\delta_{t}^{+} x \text { for } x>0 .
$$

Taking into account that $\beta_{t}$ is convex, we conclude from (9.18) that $\beta_{t}(x)$ has an asymptote of slope $\delta_{t}^{+}$as $x \rightarrow+\infty$. Let $y=\nu_{t}^{+}+\delta_{t}^{+} x$ be the equation of this asymptote. Then

$$
\nu_{t}^{+}+\delta_{t}^{+} x \leq \beta_{t}(x) \leq \nu_{t}^{+}+\delta_{t}^{+} x+o_{1}(x) \text { for } x>0
$$

where $o_{1}(x) \rightarrow 0$ as $x \rightarrow+\infty$. From (9.19) and Theorem 3.5 we get $\nu_{t}^{+} \leq \beta_{t}(0)<$ $1 / q$. In the same way we may derive from (9.12) and (7.1) that

$$
\mu_{t}^{+}+\delta_{t}^{+} x-o_{2}(x) \leq \alpha_{t}(x) \leq \mu_{t}^{+}+\delta_{t}^{+} x \text { for } x<0
$$

with $\mu_{t}^{+} \geq \alpha_{t}(0)>-1 / p$ and $o_{2}(x) \rightarrow 0$ as $x \rightarrow-\infty$.

It remains to show that $\mu_{t}^{+} \leq \nu_{t}^{+}$. Assume $\mu_{t}^{+}>\nu_{t}^{+}+4 \varepsilon$ with $\varepsilon>0$. By $(9.19)$ and (9.20),

$$
\beta_{t}(x) \leq \nu_{t}^{+}+\delta_{t}^{+} x+o_{1}(x), \quad \alpha_{t}(-x) \geq \mu_{t}^{+}-\delta_{t}^{+} x-o_{2}(-x)
$$

for every $x>0$. Hence, there exists an $x>0$ such that

$$
\beta_{t}(x) \leq \nu_{t}^{+}+\delta_{t}^{+} x+\varepsilon, \alpha_{t}(-x) \geq \mu_{t}^{+}-\delta_{t}^{+} x-\varepsilon,
$$

whence

$$
\beta_{t}(x)-\alpha_{t}(-x) \leq \nu_{t}^{+}+\delta_{t}^{+} x+\varepsilon-\mu_{t}^{+}+\delta_{t}^{+} x+\varepsilon<2 \delta_{t}^{+} x-2 \varepsilon
$$

and thus

$$
\lim _{y \rightarrow \infty}\left(\frac{\log \left(V_{t}^{0} \eta_{t}^{x} \omega\right)(y)}{\log y}+\frac{\log \left(V_{t}^{0} \eta_{t}^{-x} \omega\right)(1 / y)}{\log y}\right)<2 \delta_{t}^{+} x-2 \varepsilon
$$

The latter inequality implies the existence of an $y_{0}>1$ such that

$$
\left(V_{t}^{0} \eta_{t}^{x} \omega\right)(y)\left(V_{t}^{0} \eta_{t}^{-x} \omega\right)(1 / y) \leq y^{2 \delta_{t}^{+} x-\varepsilon}
$$

for all $y>y_{0}$. Put

$$
f_{x}(R, \omega):=\exp \left(\Delta_{t}\left(x \log \eta_{t}+\log \omega, 0, R\right)\right) .
$$

Then, by the definition of $V_{t}^{0}$,

$$
\left(V_{t}^{0} \eta_{t}^{x} \omega\right)(y)=\limsup _{R \rightarrow 0} \frac{f_{x}(R, \omega)}{f_{x}\left(y^{-1} R, \omega\right)}, \quad\left(V_{t}^{0} \eta_{t}^{-x} \omega\right)\left(\frac{1}{y}\right)=\limsup _{R \rightarrow 0} \frac{f_{-x}\left(y^{-1} R, \omega\right)}{f_{-x}(R, \omega)}
$$


for $y>1$ and hence, by (9.21),

$$
\limsup _{R \rightarrow 0}\left(\frac{f_{x}(R, \omega)}{f_{x}\left(y^{-1} R, \omega\right)} \frac{f_{-x}\left(y^{-1} R, \omega\right)}{f_{-x}(R, \omega)}\right) \leq y^{2 \delta_{t}^{+} x-\varepsilon}
$$

for $y>y_{0}$. Because

$$
\frac{f_{x}(R, \omega)}{f_{x}\left(y^{-1} R, \omega\right)} \frac{f_{-x}\left(y^{-1} R, \omega\right)}{f_{-x}(R, \omega)}=\frac{f_{2 x}(R, 1)}{f_{2 x}\left(y^{-1} R, 1\right)},
$$

it follows from (9.22) that

$$
\left(V_{t}^{0} \eta_{t}^{2 x}\right)(y)=\limsup _{R \rightarrow 0} \frac{f_{2 x}(R, 1)}{f_{2 x}\left(y^{-1} R, 1\right)} \leq y^{2 \delta_{t}^{+} x-\varepsilon} .
$$

The latter estimate shows that

$$
\beta\left(V_{t}^{0} \eta_{t}^{2 x}\right) \leq 2 \delta_{t}^{+} x-\varepsilon .
$$

On the other hand, from Lemmas 9.2 and 7.1 we get

$$
\beta\left(V_{t}^{0} \eta_{t}^{2 x}\right)=\beta\left(V_{t}^{0} \varphi_{t, 2 i x}\right)=\beta\left(W_{t} \varphi_{t, 2 i x}\right)=2 \delta_{t}^{+} x,
$$

which contradicts (9.23) and completes the proof of Theorem 3.2.

As already mentioned in Section 3, Theorem 3.3 follows from Theorem 3.2 without difficulty.

\section{INDICATOR FUNCTIONS WITH PRESCRIBED SHAPE}

This section is dedicated to the proof of Theorem 3.4.

Let $h$ and $g$ be twice continuously differentiable real-valued functions on $\mathbf{R}$ such that $h, h^{\prime}, h^{\prime \prime}, g, g^{\prime}, g^{\prime \prime}$ are bounded on $\mathbf{R}$. Also suppose that $h$ and $g$ are constant on $(-\infty, 0)$. Put

$$
\varphi(r)=h(\log (-\log r)) \log r, \quad v(r)=g(\log (-\log r)) \log r
$$

for $r \in(0,1)$ and define $\varphi(1)=v(1)=0$. Further, choose any real-valued function $b \in C^{1}[0,1]$ such that

$$
b(0)=b(1)=0, \quad 0<b(r)<2 \pi \text { for } r \in(0,1) .
$$

Pick a point $t \in \mathbf{C}$ and put

$$
\begin{aligned}
& \Gamma_{+}:=\left\{\tau=t+r e^{-i \varphi(r)}: r \in(0,1]\right\}, \\
& \Gamma_{-}:=\left\{\tau=t+r e^{-i(\varphi(r)-b(r))}: r \in(0,1]\right\} .
\end{aligned}
$$

Then $\Gamma:=\{t\} \cup \Gamma_{+} \cup \Gamma_{-}$is a Jordan curve and the function $\omega: \Gamma \backslash\{t\} \rightarrow(0, \infty)$ given by

$$
\omega(\tau):=e^{v(|\tau-t|)} \quad(\tau \in \Gamma \backslash\{t\})
$$

is a weight which is continuous on $\Gamma \backslash\{t\}$.

Lemma 10.1. $\Gamma$ is a Carleson curve.

Proof. Lemmas 4.1 and 4.2 of [5].

Lemma 10.2. The weight $\omega$ belongs to $A_{p}(\Gamma)$ if and only if

$$
-\frac{1}{p}<\liminf _{y \rightarrow+\infty}\left(g(y)+g^{\prime}(y)\right) \leq \limsup _{y \rightarrow+\infty}\left(g(y)+g^{\prime}(y)\right)<\frac{1}{q} .
$$


Proof. Lemma 5.10 and Theorem 6.3.

We now construct $h$ and $g$ in a special way.

Lemma 10.3. Given real numbers $c>0, d>0, a \in \mathbf{R}$, there exists a function $\chi \in C^{2}[0,1]$ with support in $(0,1)$ such that

$$
\max _{x \in[0,1]}\left(\chi(x)+\chi^{\prime}(x)\right)=c, \min _{x \in[0,1]}\left(\chi(x)+\chi^{\prime}(x)\right)=-d
$$

and

$$
\begin{aligned}
& \|\chi\|_{\infty} \leq 1, \quad\|a \chi\|_{\infty} \leq 1, \quad\left\|\chi^{\prime}\right\|_{\infty} \leq 2 \max \{c, d\}, \\
& \left\|\chi^{\prime \prime}\right\|_{\infty} \leq 100 \max \left\{c^{2}, d^{2},|a| c^{2},|a| d^{2}, c, d, d^{2} / c, c^{2} / d\right\} .
\end{aligned}
$$

Proof. It suffices to prove the lemma for $a \neq 0$. Choose any function $\psi \in C^{2}[0, \infty)$ such that

$$
\begin{aligned}
& \psi(x)=0 \text { for } 0 \leq x \leq \frac{1}{6}, \psi(x)=1 \text { for } x \geq \frac{2}{6}, \\
& \psi(x) \text { increases monotonically from } 0 \text { to } 1 \text { for } \frac{1}{6} \leq x \leq \frac{2}{6},
\end{aligned}
$$

and $\left\|\psi^{\prime}\right\|_{\infty}=7$. So $\psi^{\prime}(x)$ goes from 0 to 7 and then from 7 to 0 on $[1 / 6,2 / 6]$, and a little thought shows that we may choose $\psi$ so that $\left\|\psi^{\prime \prime}\right\|_{\infty}<85$. Put

$$
\varepsilon:=\min \left\{1, \frac{1}{|a|}, \frac{c}{14}, \frac{d}{14}\right\} .
$$

For $\lambda \geq 1$, define $\psi_{\varepsilon, \lambda}(x):=\varepsilon \psi(\lambda x)$. The function

$$
m(\lambda):=\max _{x \in\left[0, \frac{1}{2}\right]}\left(\psi_{\varepsilon, \lambda}(x)+\psi_{\varepsilon, \lambda}^{\prime}(x)\right)=\varepsilon \max _{x \in\left[0, \frac{1}{2}\right]}\left(\psi(\lambda x)+\lambda \psi^{\prime}(\lambda x)\right)
$$

is continuous; we have $m(1) \leq 8 \varepsilon<c$ and $m(\lambda) \geq 7 \varepsilon \lambda$. Hence, there exists a $\lambda_{1}>1$ such that $m\left(\lambda_{1}\right)=c$. The inequality $m(\lambda) \geq 7 \varepsilon \lambda$ implies that

$$
c \geq 7 \varepsilon \lambda_{1} .
$$

From (10.9) and (10.10) we obtain that

$$
\lambda_{1} \leq \frac{c}{7 \varepsilon}=\frac{1}{7} \max \left\{c,|a| c, 14, \frac{14 c}{d}\right\} .
$$

Put $\chi(x):=\psi_{\varepsilon, \lambda_{1}}(x)$ for $x \in[0,1 / 2]$. Then

$$
\min _{x \in\left[0, \frac{1}{2}\right]}\left(\chi(x)+\chi^{\prime}(x)\right)=0, \quad \max _{x \in\left[0, \frac{1}{2}\right]}\left(\chi(x)+\chi^{\prime}(x)\right)=c .
$$

By (10.9)-(10.11), the following estimates hold on [0,1/2]:

$$
\begin{aligned}
& \|\chi\|_{\infty}=\varepsilon \leq 1, \quad\|a \chi\|_{\infty}=|a| \varepsilon \leq 1, \\
& \left\|\chi^{\prime}\right\|_{\infty}=\varepsilon \lambda_{1}\left\|\psi^{\prime}\right\|_{\infty}=7 \varepsilon \lambda_{1} \leq c<2 c, \\
& \left\|\chi^{\prime \prime}\right\|_{\infty}=\varepsilon \lambda_{1}^{2}\left\|\psi^{\prime \prime}\right\|_{\infty}<85 \varepsilon \lambda_{1} \lambda_{1} \\
& \leq 85 \frac{c}{7} \frac{1}{7} \max \left\{c,|a| c, 14, \frac{14 c}{d}\right\}<28 \max \left\{c^{2},|a| c^{2}, c, \frac{c^{2}}{d}\right\} .
\end{aligned}
$$

Now define $\tilde{\psi}(x):=1-\psi(x)$ and $\tilde{\psi}_{\varepsilon, \lambda}(x):=\varepsilon \tilde{\psi}(\lambda x)$. Again

$$
\tilde{m}(\lambda):=\min _{x \in\left[0, \frac{1}{2}\right]}\left(\tilde{\psi}_{\varepsilon, \lambda}(x)+\tilde{\psi}_{\varepsilon, \lambda}^{\prime}(x)\right)=\varepsilon \min _{x \in\left[0, \frac{1}{2}\right]}\left(\tilde{\psi}(\lambda x)+\lambda \tilde{\psi}^{\prime}(\lambda x)\right)
$$


is continuous, $\tilde{m}(1) \geq-7 \varepsilon>-d$ and $\tilde{m}(\lambda) \leq \varepsilon-7 \varepsilon \lambda$ for $\lambda \geq 1$, so that there is a $\lambda_{2}>1$ satisfying $\tilde{m}\left(\lambda_{2}\right)=-d$. We have $-d=\tilde{m}\left(\lambda_{2}\right) \leq \varepsilon-7 \varepsilon \lambda_{2}$ and thus,

$$
7 \varepsilon \lambda_{2} \leq \varepsilon+d<2 d
$$

Combining (10.9) and (10.13) we get

$$
\lambda_{2} \leq \frac{2 d}{7 \varepsilon}=\frac{2}{7} \max \left\{d,|a| d, \frac{14 d}{c}, 14\right\} .
$$

Put $\chi(x)=\tilde{\psi}_{\varepsilon, \lambda_{2}}(x-1 / 2)$ for $x \in[1 / 2,1]$. Then

$$
\min _{x \in[1 / 2,1]}\left(\chi(x)+\chi^{\prime}(x)\right)=-d, \max _{x \in[1 / 2,1]}\left(\chi(x)+\chi^{\prime}(x)\right)=\varepsilon \leq c .
$$

From (10.12) and (10.15) we obtain (10.6). Taking into account (10.9), (10.13), (10.14) we see that on $[1 / 2,1]$ the following estimates are valid:

$$
\begin{aligned}
& \|\chi\|_{\infty}=\varepsilon \leq 1, \quad\|a \chi\|_{\infty}=|a| \varepsilon \leq 1, \\
& \left\|\chi^{\prime}\right\|_{\infty}=\varepsilon \lambda_{2}\left\|\psi^{\prime}\right\|_{\infty}=7 \varepsilon \lambda_{2}<2 d, \\
& \left\|\chi^{\prime \prime}\right\|_{\infty}=\varepsilon \lambda_{2}^{2}\left\|\psi^{\prime \prime}\right\|_{\infty}<85 \varepsilon \lambda_{2} \lambda_{2} \\
& \leq 85 \frac{2 d}{7} \frac{2}{7} \max \left\{d,|a| d, \frac{14 d}{c}, 14\right\}<100 \max \left\{d^{2},|a| d^{2}, \frac{d^{2}}{c}, d\right\} .
\end{aligned}
$$

We have so defined $\chi$ on $[0,1 / 2] \cup[1 / 2,1]=[0,1]$ and have shown that $\chi$ possesses all the properties required.

For each $j=1,2,3, \ldots$ choose real numbers $c_{j}>0, d_{j}>0, a_{j} \in \mathbf{R}$, denote by $\chi_{j} \in C^{2}[0,1]$ the corresponding function from Lemma 10.3 with $c=c_{j}, d=d_{j}, a=$ $a_{j}$, and extend $\chi_{j}$ periodically (with period 1 ) to all of $\mathbf{R}$.

Let $I_{n}:=[n, n+1)$ and denote by $R_{j}(j=1,2,3, \ldots)$ the union of the sets in the $j$ th row of the matrix

$$
\left(\begin{array}{ccccc}
I_{0} & I_{1} & I_{3} & I_{6} & \ldots \\
I_{2} & I_{4} & I_{7} & \ldots & \ldots \\
I_{5} & I_{8} & \ldots & \ldots & \ldots \\
I_{9} & \ldots & \ldots & \ldots & \ldots \\
\ldots & \ldots & \ldots & \ldots & \ldots
\end{array}\right)
$$

Obviously, $R_{i} \cap R_{j}=\emptyset$ for $i \neq j$ and $\bigcup_{j=1}^{\infty} R_{j}=[0, \infty)$. Finally, pick numbers $\lambda \in \mathbf{R}, \delta \in \mathbf{R}$ and define

$$
\begin{aligned}
& g(y)=\lambda+a_{j} \chi_{j}(y) \text { for } y \in R_{j}, g(y)=\lambda \text { for } y<0, \\
& h(y)=\delta+\chi_{j}(y) \text { for } y \in R_{j}, g(y)=\delta \text { for } y<0 .
\end{aligned}
$$

From Lemma 10.3 we infer that $g$ and $h$ are twice continuously differentiable and that $g, g^{\prime}, g^{\prime \prime}, h, h^{\prime}, h^{\prime \prime}$ are bounded on $\mathbf{R}$ whenever

$$
\sup _{j} \max \left\{c_{j}, d_{j}, \frac{d_{j}}{c_{j}}, \frac{c_{j}}{d_{j}},\left|a_{j}\right| c_{j},\left|a_{j}\right| d_{j}\right\}<\infty .
$$

Hence, if (10.18) holds, then the curve $\Gamma=\{t\} \cup \Gamma_{-} \cup \Gamma_{+}$given by (10.1), (10.2), (10.3), (10.17) is a Carleson Jordan curve by virtue of Lemma 10.1. Define the weight $\omega$ by (10.1), (10.4), (10.16). 
Lemma 10.4. Suppose (10.18) is satisfied. Then $\omega \in A_{p}(\Gamma)$ if and only if

$$
-\frac{1}{p}<\lambda+\inf _{j} \min \left\{c_{j} a_{j},-d_{j} a_{j}\right\} \leq \lambda+\sup _{j} \max \left\{c_{j} a_{j},-d_{j} a_{j}\right\}<\frac{1}{q} .
$$

Proof. By (10.6) and the construction of the function $g$,

$$
\begin{aligned}
& \limsup _{y \rightarrow+\infty}\left(g(y)+g^{\prime}(y)\right)=\sup _{j} \max _{y \in R_{j}}\left(g(y)+g^{\prime}(y)\right) \\
& =\sup _{j} \max _{x \in[0,1]}\left(\lambda+a_{j} \chi_{j}(x)+a_{j} \chi_{j}^{\prime}(x)\right)=\sup _{j} \max \left\{\lambda+a_{j} c_{j}, \lambda-a_{j} d_{j}\right\}
\end{aligned}
$$

and analogously,

$$
\liminf _{y \rightarrow+\infty}\left(g(y)+g^{\prime}(y)\right)=\inf _{j} \min \left\{\lambda+a_{j} c_{j}, \lambda-a_{j} d_{j}\right\} .
$$

The assertion thus follows from Lemma 10.2.

Lemma 10.5. Suppose (10.18) and (10.19) hold. Then $W_{t} \varphi_{t, \gamma} \omega$ and $W_{t}^{0} \varphi_{t, \gamma} \omega$ are regular and

$$
\begin{aligned}
& \alpha\left(W_{t}^{0} \varphi_{t, \gamma} \omega\right)=\operatorname{Re} \gamma+\lambda+\delta \operatorname{Im} \gamma+A(\operatorname{Im} \gamma), \\
& \beta\left(W_{t}^{0} \varphi_{t, \gamma} \omega\right)=\operatorname{Re} \gamma+\lambda+\delta \operatorname{Im} \gamma+B(\operatorname{Im} \gamma)
\end{aligned}
$$

with

$$
\begin{aligned}
& A(x):=\inf _{j} \min \left\{c_{j} a_{j}+c_{j} x,-d_{j} a_{j}-d_{j} x\right\}, \\
& B(x):=\sup _{j} \max \left\{c_{j} a_{j}+c_{j} x,-d_{j} a_{j}-d_{j} x\right\} .
\end{aligned}
$$

Proof. For $x \in(0,1]$, the value $\left(W_{t} \varphi_{t, \gamma} \omega\right)(x)$ equals

$$
\sup _{R>0} \frac{\max _{|\tau-t|=x R}|\tau-t|^{\operatorname{Re} \gamma} e^{-\operatorname{Im} \gamma \arg (\tau-t)} e^{v(|\tau-t|)}}{\min _{|\tau-t|=R}|\tau-t|^{\operatorname{Re} \gamma} e^{-\operatorname{Im} \gamma \arg (\tau-t)} e^{v(|\tau-t|)}} .
$$

We have

$$
\begin{aligned}
& \max _{|\tau-t|=x R} e^{-\operatorname{Im} \gamma \arg (\tau-t)}=\max \left\{e^{\operatorname{Im} \gamma \varphi(x R)}, e^{\operatorname{Im} \gamma(\varphi(x R)-b(x R))}\right\}, \\
& \min _{|\tau-t|=R} e^{-\operatorname{Im} \gamma \arg (\tau-t)}=\min \left\{e^{\operatorname{Im} \gamma \varphi(R)}, e^{\operatorname{Im} \gamma(\varphi(R)-b(R))}\right\},
\end{aligned}
$$

and hence (10.24) is equal to

$$
\begin{aligned}
& x^{\operatorname{Re} \gamma} \sup _{R>0} e^{v(x R)-v(R)} e^{\operatorname{Im} \gamma(\varphi(x R)-\varphi(R))} e^{\operatorname{Im} \gamma b(R)} \text { for } \operatorname{Im} \gamma \geq 0, \\
& x^{\operatorname{Re} \gamma} \sup _{R>0} e^{v(x R)-v(R)} e^{\operatorname{Im} \gamma(\varphi(x R)-\varphi(R))} e^{-\operatorname{Im} \gamma b(x R)} \text { for } \operatorname{Im} \gamma \leq 0 .
\end{aligned}
$$

Since $b, h, h^{\prime}, h^{\prime \prime}, g, g^{\prime}, g^{\prime \prime}$ are bounded, one can verify as in the proof of Proposition 5.7 that $\log \left(W_{t} \varphi_{t, \gamma} \omega\right)(x)$ is bounded in a left semi-neighborhood of $x=1$ (see the proof of Lemma 4.2 of [5] for details). Analogously one can show boundedness in a right semi-neighborhood of $x=1$. Thus, $W_{t} \varphi_{t, \gamma} \omega$ is regular.

Now Theorem 6.3 implies that $W_{t}^{0} \varphi_{t, \gamma} \omega$ is also regular. Since $\left(W_{t}^{0} \varphi_{t, \gamma} \omega\right)(x)$ equals (10.25), (10.26) with " $\sup _{R>0}$ " replaced by "lim $\sup _{R \rightarrow 0}$ ", we get

$$
\left(W_{t}^{0} \varphi_{t, \gamma} \omega\right)(x)=x^{\operatorname{Re} \gamma} \limsup _{R \rightarrow 0} e^{v(x R)-v(R)+\operatorname{Im} \gamma(\varphi(x R)-\varphi(R))}
$$


and hence $\left(W_{t}^{0} \varphi_{t, \gamma} \omega\right)(x)=\left(W_{t}^{0} \psi\right)(x)$ with $\psi(\tau)=e^{F(|\tau-t|)}$ and

$$
\begin{aligned}
F(r) & :=\operatorname{Re} \gamma \log r+v(r)+\operatorname{Im} \gamma \varphi(r) \\
& =[\operatorname{Re} \gamma+g(\log (-\log r))+\operatorname{Im} \gamma h(\log (-\log r))] \log r .
\end{aligned}
$$

Thus, by Lemma 5.10,

$$
\alpha\left(W_{t}^{0} \varphi_{t, \gamma} \omega\right)=\operatorname{Re} \gamma+\liminf _{y \rightarrow+\infty}\left[g(y)+g^{\prime}(y)+\operatorname{Im} \gamma\left(h(y)+h^{\prime}(y)\right)\right]
$$

which gives (10.20), (10.22) as in the proof of Lemma 10.4. In a similar way one obtains (10.21), (10.23).

Proof of Theorem 3.4. Consider the parallelogram $\mathbf{P}$ with the vertices

$$
x_{t}^{-}+i \alpha_{t}\left(x_{t}^{-}\right), \quad x_{t}^{-}+i \beta_{t}\left(x_{t}^{-}\right), \quad x_{t}^{+}+i \alpha_{t}\left(x_{t}^{+}\right), x_{t}^{+}+i \beta_{t}\left(x_{t}^{+}\right)
$$

and let the diagonals of this parallelogram have the equations $y=\mu_{1}+\delta_{1} x$ and $y=\mu_{2}+\delta_{2} x$. From (P4) and (P2) we infer that

(10.27) $-1 / p<\alpha_{t}(0) \leq \mu_{1} \leq \beta_{t}(0)<1 / q, \quad-1 / p<\alpha_{t}(0) \leq \mu_{2} \leq \beta_{t}(0)<1 / q$.

Without loss of generality assume that $\delta_{1}<\delta_{2}$. We extend $\alpha_{t}$ and $\beta_{t}$ to functions $\alpha$ and $\beta$ on all of $\mathbf{R}$ as follows:

$$
\begin{aligned}
& \alpha(x):=\mu_{2}+\delta_{2} x \text { and } \beta(x):=\mu_{1}+\delta_{1} x \text { for } x<x_{t}^{-}, \\
& \alpha(x):=\alpha_{t}(x) \text { and } \beta(x):=\beta_{t}(x) \text { for } x_{t}^{-} \leq x \leq x_{t}^{+}, \\
& \alpha(x):=\mu_{1}+\delta_{1} x \text { and } \beta(x):=\mu_{2}+\delta_{2} x \text { for } x>x_{t}^{+} .
\end{aligned}
$$

By (P3) and (P4), $\alpha$ is concave, $\beta$ is convex, and the straight lines given by $y=$ $\mu_{1}+\delta_{1} x$ and $y=\mu_{2}+\delta_{2} x$ separate the convex regions $\{x+i y \in \mathbf{C}: y<\alpha(x)\}$ and $\{x+i y \in \mathbf{C}: y>\beta(x)\}$.

Suppose for a moment that we have numbers $c_{j}, d_{j}, a_{j}(j=1,2,3, \ldots)$ and numbers $\lambda \in \mathbf{R}, \delta \in \mathbf{R}$ satisfying (10.18) and (10.19) such that

$$
\alpha(x)=\lambda+\delta x+A(x), \beta(x)=\lambda+\delta x+B(x)
$$

where $A(x)$ and $B(x)$ are given by (10.22) and (10.23). Defining $\Gamma$ and $\omega$ as above, we get

$$
\alpha\left(W_{t}^{0} \varphi_{t, y+i x} \omega\right)=y+\alpha(x), \beta\left(W_{t}^{0} \varphi_{t, y+i x} \omega\right)=y+\beta(x)
$$

from Lemma 10.5. The indicator functions of $\Gamma, p, \omega$ at $t \in \Gamma$ are

$$
\tilde{\alpha}_{t}(x):=\alpha\left(V_{t}^{0} \eta_{t}^{x} \omega\right), \quad \tilde{\beta}_{t}(x):=\beta\left(V_{t}^{0} \eta_{t}^{x} \omega\right) .
$$

Theorem 6.3 implies that

$$
N_{t}=\left\{y+i x \in \mathbf{C}:-\frac{1}{p}<y+\alpha(x) \leq y+\beta(x)<\frac{1}{q}\right\},
$$

while Theorem 3.5 tells us that

$$
N_{t}=\left\{y+i x \in \mathbf{C}:-\frac{1}{p}<y+\tilde{\alpha}_{t}(x) \leq y+\tilde{\beta}_{t}(x)<\frac{1}{q}\right\} .
$$

From property (P1) and (10.29) we deduce that $N_{t}$ is bounded. So (10.30) yields that the equation $\tilde{\beta}_{t}(x)-\tilde{\alpha}_{t}(x)=1$ has exactly two solutions, $\tilde{x_{t}^{-}}<0$ and $\tilde{x_{t}^{+}}>0$. Since (10.29) and (10.30) are one and the same set, it follows that $\tilde{x_{t}^{-}}=x_{t}^{-}, \tilde{x_{t}^{+}}=$ $x_{t}^{+}$, and

$$
\tilde{\alpha}_{t}(x)=\alpha(x)=\alpha_{t}(x), \quad \tilde{\beta}_{t}(x)=\beta(x)=\beta_{t}(x)
$$


for all $x \in\left[x_{t}^{-}, x_{t}^{+}\right]$, which completes the proof.

So let us construct the numbers $c_{j}, d_{j}, a_{j}$. Denote by $x_{0}+i y_{0}$ the intersection of the diagonals of the parallelogram P. From (P3) and (P4) we see that $\alpha\left(x_{0}\right)=$ $y_{0}=\beta\left(x_{0}\right)$ happens if and only if

$$
\alpha(x)=\min \left\{\mu_{1}+\delta_{1} x, \mu_{2}+\delta_{2} x\right\}, \quad \beta(x)=\max \left\{\mu_{1}+\delta_{1} x, \mu_{2}+\delta_{2} x\right\} .
$$

Choose any $\delta$ such that $\delta_{1}<\delta<\delta_{2}$, and put

$$
c=\delta_{2}-\delta, d=\delta-\delta_{1}, a=\frac{\mu_{2}-\mu_{1}}{c+d}, \lambda=\frac{c \mu_{1}+d \mu_{2}}{c+d}
$$

and $c_{j}=c, d_{j}=d, a_{j}=a$ for all $j$. Then $c_{j}>0, d_{j}>0$, an easy computation along with (10.31) shows that (10.28) holds, and (10.18) is obviously satisfied. Since $\lambda+c a=\mu_{2}$ and $\lambda-d a=\mu_{1}$, we obtain from (10.27) that (10.19) is also satisfied. Thus, the case where the graphs of $y=\alpha(x)$ and $y=\beta(x)$ have the point $x_{0}+i y_{0}$ in common is settled.

Suppose $\alpha\left(x_{0}\right)<\beta\left(x_{0}\right)$. Then there is a line $y=\lambda+\delta x$ which separates the two convex sets $\{x+i y \in \mathbf{C}: y \geq \beta(x)\}$ and $\{x+i y \in \mathbf{C}: y \leq \alpha(x)\}$. Thus, if we put

$$
\alpha_{0}(x):=\alpha(x)-\delta x-\lambda, \quad \beta_{0}(x):=\beta(x)-\delta x-\lambda,
$$

then

$$
\alpha^{*}:=\sup _{x \in \mathbf{R}} \alpha_{0}(x)<0<\inf _{x \in \mathbf{R}} \beta_{0}(x)=: \beta^{*} .
$$

Clearly, $\alpha_{0}$ is concave and $\beta_{0}$ is convex. Moreover, we have $\delta_{1}<\delta<\delta_{2}$ and hence, $c:=\delta_{2}-\delta>0$ and $d:=\delta-\delta_{1}>0$. Obviously,

$$
\begin{aligned}
& \mu_{2}-\lambda+c x \leq \beta_{0}(x) \leq \mu_{2}-\lambda+c x+o_{+}(1), \\
& \mu_{1}-\lambda-d x \leq \beta_{0}(x) \leq \mu_{1}-\lambda-d x+o_{-}(1), \\
& \mu_{1}-\lambda-d x \geq \alpha_{0}(x) \geq \mu_{1}-\lambda-d x-o_{+}(1), \\
& \mu_{2}-\lambda+c x \geq \alpha_{0}(x) \geq \mu_{2}-\lambda+c x-o_{-}(1),
\end{aligned}
$$

where $o_{ \pm}(1)$ denote nonnegative functions which vanish in a neighborhood of $\pm \infty$. Put

$$
\xi_{1}:=-\left(\lambda-\mu_{1}\right) / d, \quad \xi_{2}:=\left(\lambda-\mu_{2}\right) / c,
$$

i.e. let $\xi_{1}$ and $\xi_{2}$ be the points at which the lines $y=\mu_{1}-\lambda-d x$ and $y=\mu_{2}-\lambda+c x$ meet the real axis.

The set $\mathbf{Q}$ of all rational numbers is countable. Let $\left\{a_{j}\right\}_{j=1}^{\infty}=\mathbf{Q}$. We construct the sequences $\left\{c_{j}\right\}_{j=1}^{\infty}$ and $\left\{d_{j}\right\}_{j=1}^{\infty}$ as follows:

for $-\infty<-a_{j} \leq \xi_{2}$ (resp. $\xi_{2}<-a_{j}<+\infty$ ) let $c_{j}>0$ be the number defined by requiring that $y \geq c_{j} a_{j}+c_{j} x$ (resp. $y \leq c_{j} a_{j}+c_{j} x$ ) is a supporting half-plane to $y \geq \beta_{0}(x)$ (resp. $y \leq \alpha_{0}(x)$ );

for $-\infty<-a_{j} \leq \xi_{1}$ (resp. $\xi_{1}<-a_{j}<+\infty$ ) define $d_{j}>0$ as the number for which $y \leq-d_{j} a_{j}-d_{j} x$ (resp. $y \geq-d_{j} a_{j}-d_{j} x$ ) is a supporting half-plane to $y \leq \alpha_{0}(x)$ (resp. $y \geq \beta_{0}(x)$ ).

It is clear that $0<c_{j} \leq c$ for all $j$ and that $c_{j} \rightarrow c$ as $-a_{j} \rightarrow \xi_{2}$. This proves that $\sup _{j} c_{j}=c<\infty$. Analogously, $0<d_{j} \leq d$ for all $j, d_{j} \rightarrow d$ as $-a_{j} \rightarrow \xi_{1}$, and 
thus $\sup _{j} d_{j}=d<\infty$. The lines $y=c_{j} a_{j}+c_{j} x$ always meet the imaginary axis between $i \alpha_{0}(0)$ and $i \beta_{0}(0)$, whence, by (10.27),

$(10.33)$

$$
-\frac{1}{p}<\alpha_{t}(0)=\alpha(0)=\lambda+\alpha_{0}(0) \leq \lambda+c_{j} a_{j} \leq \lambda+\beta_{0}(0)=\beta(0)=\beta_{t}(0)<\frac{1}{q} .
$$

Equally,

$$
-1 / p<\alpha_{t}(0) \leq \lambda-d_{j} a_{j} \leq \beta_{t}(0)<1 / q,
$$

which shows that (10.19) is satisfied. From (10.33) and (10.34) we also get

$$
\sup _{j}\left|a_{j}\right| c_{j}<\infty, \sup _{j}\left|a_{j}\right| d_{j}<\infty .
$$

Thus, the proof of (10.18) will be complete once we have shown that $\sup _{j}\left(d_{j} / c_{j}\right)<$ $\infty$ and $\sup _{j}\left(c_{j} / d_{j}\right)<\infty$. We now prove these estimates.

If $-a_{j} \rightarrow-\infty$, then $c_{j} a_{j}$, the imaginary part of the intersection of the line $y=c_{j} a_{j}+c_{j} x$ with the imaginary axis, goes to the minimal value $\beta^{*}:=\inf _{x \in \mathbf{R}} \beta_{0}(x)$ of $\beta_{0}$. A similar argument shows that $-d_{j} a_{j} \rightarrow \alpha^{*}:=\sup _{x \in \mathbf{R}} \alpha_{0}(x)$ as $-a_{j} \rightarrow-\infty$. It follows from (10.32) that

$$
\frac{d_{j}}{c_{j}}=\frac{d_{j} a_{j}}{c_{j} a_{j}} \rightarrow \frac{-\alpha^{*}}{\beta^{*}}>0 \text { as }-a_{j} \rightarrow-\infty .
$$

In the same manner one can show that

$$
\frac{d_{j}}{c_{j}}=\frac{d_{j} a_{j}}{c_{j} a_{j}} \rightarrow \frac{\beta^{*}}{-\alpha^{*}}>0 \text { as }-a_{j} \rightarrow+\infty .
$$

Hence, there exists a rational number $M:=a_{j_{1}}>\max \left\{\left|\xi_{1}\right|,\left|\xi_{2}\right|\right\}$ such that

$$
\frac{d_{j}}{c_{j}}<2 \max \left\{\frac{-\alpha^{*}}{\beta^{*}}, \frac{\beta^{*}}{-\alpha^{*}}\right\} \text { whenever }\left|a_{j}\right| \geq M
$$

Let $a_{j_{2}}:=-M$. If $-a_{j}$ changes from $-a_{j}=-a_{j_{1}}=-M$ to $-a_{j}=-a_{j_{2}}=+M$, then $c_{j}$ first increases from $c_{j_{1}}$ to $c$ and then decreases from $c$ to $c_{j_{2}}$. Consequently, for $\left|a_{j}\right|<M$ we have $c_{j} \geq \min \left\{c_{j_{1}}, c_{j_{2}}\right\}$ and thus

$$
\frac{d_{j}}{c_{j}} \leq d \max \left\{\frac{1}{c_{j_{1}}}, \frac{1}{c_{j_{2}}}\right\} \text { for }\left|a_{j}\right|<M .
$$

Combining (10.35) and (10.36) we get $\sup _{j}\left(d_{j} / c_{j}\right)<\infty$. In the same way one can show that $\sup _{j}\left(c_{j} / d_{j}\right)<\infty$. At this point the proof of (10.18) and (10.19) is complete.

We now show that $\beta_{0}(x)=B(x)$. Since $\beta_{0}(x) \geq c_{j} a_{j}+c_{j} x$ and $\beta_{0}(x) \geq-d_{j} a_{j}-$ $d_{j} x$ for all $j$ and all $x$, it follows that

$$
\beta_{0}(x) \geq \sup _{j} \max \left\{c_{j} a_{j}+c_{j} x,-d_{j} a_{j}-d_{j} x\right\}=B(x)
$$

for all $x$. Assume there is a $\xi_{0} \in \mathbf{R}$ such that $\beta_{0}\left(\xi_{0}\right)>B\left(\xi_{0}\right)$. A convex function is not differentiable at at most countably many points (see e.g. Theorem 25.3 of $[27])$. Thus, there is a $\xi$ in a neighborhood of $\xi_{0}$ such that

$$
\beta_{0}(\xi)>B(\xi)
$$

and both $\beta_{0}$ and $B$ are differentiable at $\xi$. The supporting line to $y=\beta_{0}(x)$ through the point $\xi+i \beta_{0}(\xi)$ is the tangent

$$
y=\beta_{0}(\xi)+\beta_{0}^{\prime}(\xi)(x-\xi) .
$$


Suppose $\beta_{0}^{\prime}(\xi)>0$. If $\beta_{0}(\xi)+\beta_{0}^{\prime}(\xi)(x-\xi)=0$ has a rational solution $x$, then there exists a number $j$ such that $(10.39)$ is the line $y=c_{j} a_{j}+c_{j} x$ with $c_{j}=\beta_{0}^{\prime}(\xi), a_{j} \in$ $\mathbf{Q},-a_{j} \leq \xi_{2}$. It follows that

$$
\beta_{0}(\xi)=c_{j} a_{j}+c_{j} \xi \leq \sup _{j} \max \left\{c_{j} a_{j}+c_{j} \xi,-d_{j} a_{j}-d_{j} \xi\right\}=B(\xi),
$$

which contradicts (10.38). If the solution $x^{*}:=\xi-\beta_{0}(\xi) / \beta_{0}^{\prime}(\xi)$ of the equation $\beta_{0}(\xi)+\beta_{0}^{\prime}(\xi)(x-\xi)=0$ is irrational, we may choose

$$
-a_{j_{n}} \in\left(-\infty, x^{*}\right) \cap \mathbf{Q} \subset\left(-\infty, \xi_{2}\right) \cap \mathbf{Q}
$$

such that $-a_{j_{n}}$ approaches $x^{*}$ monotonically. Since $c_{j_{n}}$ is monotonically increasing and $c_{j_{n}} \leq \beta_{0}^{\prime}(\xi)$, there is a $c^{*} \leq \beta_{0}^{\prime}(\xi)$ such that $c_{j_{n}} \rightarrow c^{*}$. If $c^{*}<\beta_{0}^{\prime}(\xi)$, then the line $y=c_{j_{n}} a_{j_{n}}+c_{j_{n}} x$ cannot be a supporting line of the curve $y=\beta_{0}(x)$ whenever $-a_{j_{n}}$ is close enough to $x^{*}$ (recall (10.32)). Hence $c^{*}=\beta_{0}^{\prime}(\xi)$ and consequently,

$$
c_{j_{n}} \rightarrow \beta_{0}^{\prime}(\xi), c_{j_{n}} a_{j_{n}} \rightarrow \beta_{0}^{\prime}(\xi)\left(-x^{*}\right)=\beta_{0}(\xi)-\xi \beta_{0}^{\prime}(\xi) .
$$

It follows that $\beta_{0}(\xi)=\lim _{n \rightarrow \infty}\left(c_{j_{n}} a_{j_{n}}+c_{j_{n}} \xi\right)$, and thus there is an $n_{0}$ such that

$$
\beta_{0}(\xi)<c_{j_{n_{0}}} a_{j_{n_{0}}}+c_{j_{n_{0}}} \xi+\left(\beta_{0}(\xi)-B(\xi)\right) / 2
$$

From (10.40) and (10.37) we obtain

$$
\beta_{0}(\xi)<B(\xi)+\left(\beta_{0}(\xi)-B(\xi)\right) / 2=\left(B(\xi)+\beta_{0}(\xi)\right) / 2 \leq \beta_{0}(\xi),
$$

which is a contradiction. Analogously one can dispose of the case $\beta_{0}^{\prime}(\xi)<0$. So let $\beta_{0}^{\prime}(\xi)=0$. Choosing $-a_{j_{n}} \in\left(-\infty, \xi_{2}\right)$ such that $-a_{j_{n}} \rightarrow-\infty$ and taking into account that, by convexity, the function $\beta_{0}$ assumes its minimum at $\xi$, we see as above that $c_{j_{n}} a_{j_{n}}+c_{j_{n}} \xi \rightarrow \beta_{0}(\xi)$, which again results in the contradiction (10.41).

So we have proved that $\beta_{0}(x)=B(x)$ for all $x \in \mathbf{R}$. In the same way one can show that $\alpha_{0}(x)=A(x)$ for all $x \in \mathbf{R}$. This gives (10.28).

\section{REFERENCES}

1. A. V. Aizenshtat, Yu. I. Karlovich, and G. S. Litvinchuk, On the defect numbers of the Kveselava-Vekua operator with discontinuous derivative of the shift, Soviet Math. Dokl. 43 (1991), 633-638. MR 93d:30008

2. A. V. Aizenshtat, Yu. I. Karlovich, and G. S. Litvinchuk, The method of conformal gluing for the Haseman boundary value problem on an open contour, Complex Variables 28 (1996), 313-346.

3. C. Bennett and R. Sharpley, Interpolation of operators, Academic Press, Boston, 1988. MR 89e: 46001

4. A. Böttcher and Yu. I. Karlovich, Toeplitz and singular integral operators on Carleson curves with logarithmic whirl points, Integral Equations and Operator Theory 22 (1995), 127-161. MR 96j: 47022

5. A. Böttcher and Yu. I. Karlovich, Toeplitz and singular integral operators on general Carleson Jordan curves, Operator Theory: Advances and Applications 90 (1996), 119-152. MR 97k:47024

6. A. Böttcher and B. Silbermann, Analysis of Toeplitz operators, Akademie-Verlag, Berlin 1989 and Springer-Verlag, Berlin, Heidelberg, New York 1990. MR 92e:47001

7. D. Boyd, Indices for the Orlicz spaces, Pacific J. Math. 38 (1971), 315-323. MR 46:6008

8. L. A. Coburn, Weyl's theorem for non-normal operators, Michigan Math. J. 13 (1966), 285286. MR 34:1846

9. E. A. Danilov, The Riemann boundary value problem on contours with unbounded distortion, Cand. Dissertation, Odessa 1984 [Russian].

10. G. David, L'integrale de Cauchy sur le courbes rectifiables, Prepublication Univ. Paris-Sud, Dept. Math. 82T05, 1982.

11. G. David, Opérateurs intégraux singuliers sur certaines courbes du plan complexe, Ann. Sci. École Norm. Super 17 (1984), 157-189. 
12. R. G. Douglas, Banach algebra techniques in operator theory, Academic Press, New York 1972. MR 50:14335

13. E. M. Dynkin, Methods of the theory of singular integrals (Hilbert transform and CalderonZygmund theory), In: Itogi Nauki Tekh., Sovr. Probl. Matem., Fund. Napravl., vol. 15, Moscow 1987, pp. 197-292 [Russian]. CMP 20:04

14. E. M. Dynkin, Methods of the theory of singular integrals II (Littlewood-Paley theory and its applications), In: Itogi Nauki Tekh., Sovr. Probl. Matem., Fund. Napravl., vol. 42, Moscow 1989, pp. 105-198 [Russian]. MR 91j:42015

15. E. M. Dynkin and B. P. Osilenker, Weighted norm estimates for singular integrals and their applications, J. Sov. Math. 30 (1985), 2094-2154 [Russian original: Itogi Nauki Tekh., Ser. Mat. Anal. 21 (1983), 42-129].

16. T. Finck, S. Roch, and B. Silbermann, Two projections theorems and symbol calculus for operators with massive local spectra, Math. Nachr. 162 (1993), 167-185. MR 94h:47083

17. J. B. Garnett, Bounded analytic functions, Academic Press, New York 1981. MR 83g:30037

18. I. Gohberg, On an application of the theory of normed rings to singular integral equations, Uspehi Matem. Nauk 7 (1952), 149-156 [Russian]. MR 14:54a

19. I. Gohberg and N. Krupnik, Singular integral operators with piecewise continuous coefficients and their symbols, Math. USSR Izv. 5 (1971), 955-979.

20. I. Gohberg and N. Krupnik, One-dimensional linear singular integral equations, Vols. I and II, Birkhäuser Verlag, Basel, Boston, Berlin 1992 [Russian original: Shtiintsa, Kishinev 1973].

21. I. Gohberg and N. Krupnik, Extension theorems for Fredholm and invertibility symbols, Integral Equations and Operator Theory 16 (1993), 514-529.

22. I. Gohberg, N. Krupnik, and I. M. Spitkovsky, Banach algebras of singular integral operators with piecewise continuous coefficients, General contour and weight. Integral Equations and Operator Theory 17 (1993), 322-337. MR 94f:47057

23. E. Hille and R. S. Phillips, Functional analysis and semi-groups, Amer. Math. Soc. Coll. Publ., vol. 31, revised edition, Providence, R.I, 1957.

24. R. Hunt, B. Muckenhoupt, and R. Wheeden, Weighted norm inequalities for the conjugate function and Hilbert transform, Trans. Amer. Math. Soc. 176 (1973), 227-251. MR 47:701

25. S. G. Krein, Yu. I. Petunin, and E. M. Semenov, Interpolation of linear operators, Transl. Math. Monogr. 54, Amer. Math. Soc., Providence, R.I., 1982 [Russian original: Nauka, Moscow, 1978]. MR 84j:46103

26. V. A. Paatashvili and G. A. Khuskivadze, On the boundedness of the Cauchy singular integral on Lebesgue spaces in the case of non-smooth contours, Trudy Tbilisk. Mat. Inst. AN GSSR 69 (1982), 93-107 [Russian].

27. R. T. Rockafellar, Convex analysis, Princeton University Press, Princeton 1970. MR 43:445

28. R. K. Seifullayev, The Riemann boundary value problem on non-smooth open curves, Matem. Sb. 112 (1980), 147-161 [Russian] (English transl. in Math. USSR Sb. 40 (1981)).

29. I. B. Simonenko, The Riemann boundary value problem with measurable coefficients, Dokl. Akad. Nauk SSSR 135 (1960), 538-541 [Russian].

30. I. B. Simonenko, Some general questions of the theory of the Riemann boundary value problem, Math. USSR Izv. 2 (1968), 1091-1099.

31. I. B. Simonenko, On the factorization and local factorization of measurable functions, Soviet Math. Dokl. 21 (1980), 271-274.

32. I. B. Simonenko, Stability of weight properties of functions with respect to the singular integral, Matem. Zametki 33 (1983), 409-416 [Russian].

33. I. M. Spitkovsky, Singular integral operators with PC symbols on the spaces with general weights, J. Funct. Anal. 105 (1992), 129-143. MR 93d:47057

34. H. Widom, Singular integral equations in $L^{p}$, Trans. Amer. Math. Soc. 97 (1960), 131-160. MR 22:9830

Faculty of Mathematics, Tech. Univ. Chemnitz-Zwickau, D-09107 Chemnitz, Germany E-mail address: aboettch@mathematik.tu-chemnitz.de

Faculty of Mathematics, Tech. Univ. Chemnitz-Zwickau, D-09107 Chemnitz, Germany Current address: Ukrainian Academy of Sciences, Marine Hydrophysical Institute, Hydroacoustic Department, Preobrazhenskaya Street 3, 270100 Odessa, Ukraine

E-mail address: karlik@paco.net 